
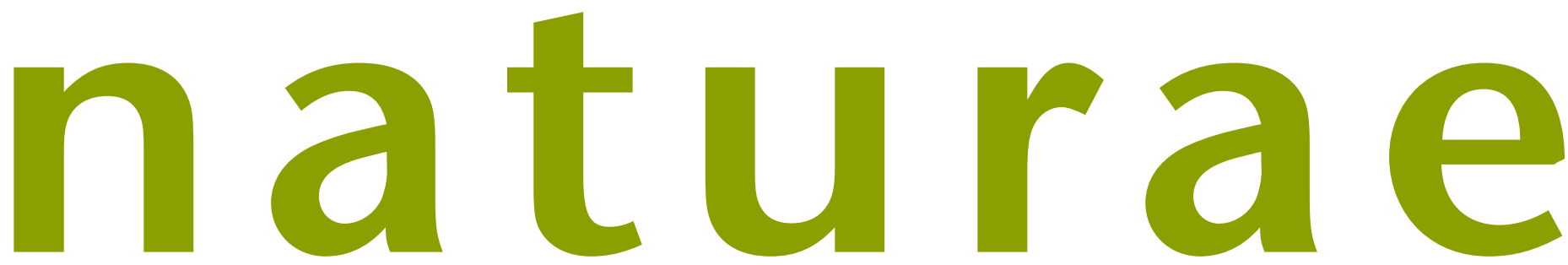

$2022 \cdot 4$

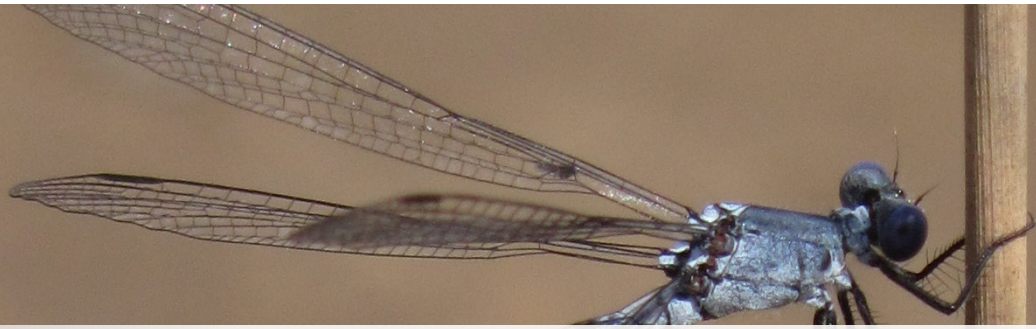

Les Arthropodes terrestres dans les études d'impact: limites actuelles et propositions pour une meilleure prise en compte des enjeux de conservation

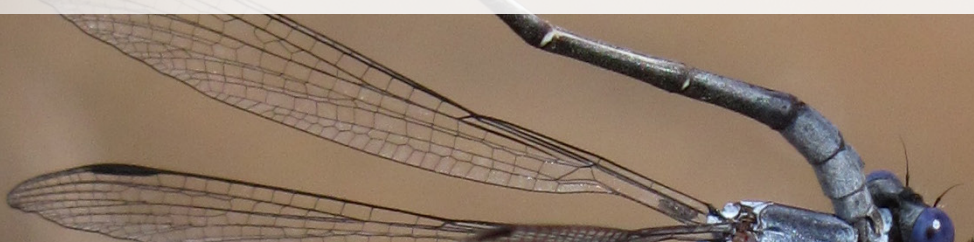

Étienne IORIO, François DUSOULIER, Fabien SOLDATI, Franck NOËL, Jean-Alain GUILLOTON, Guillaume DOUCET, Philippe PONEL, Pascal DUPONT, Régis KRIEG-JACQUIER, Stéphane CHEMIN, Pierre TILLIER \& Julien TOUROULT

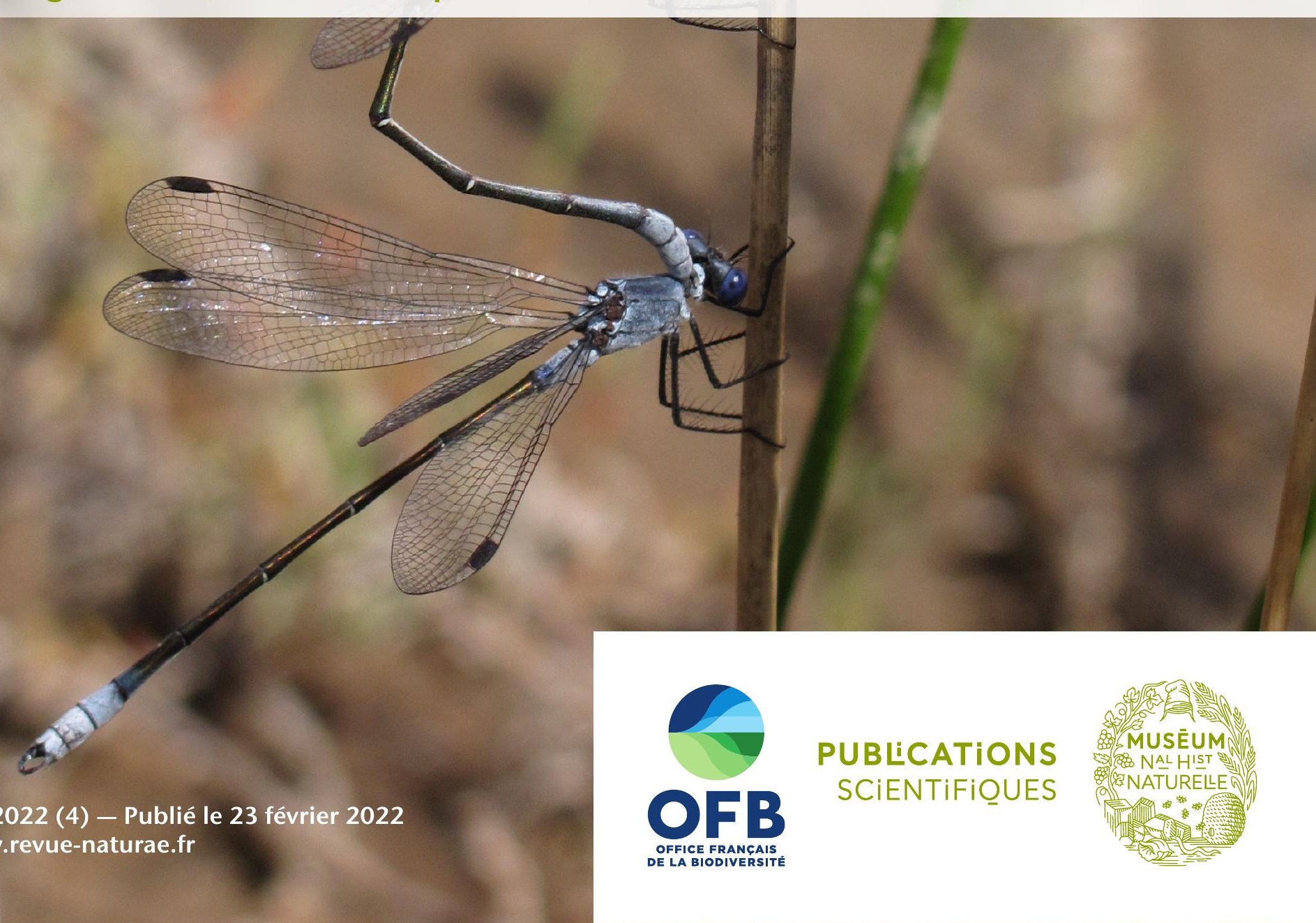


DiRECTEUR DE LA PUBLICATION / PUBLICATION DIRECTOR: Bruno David,

Président du Muséum national d'Histoire naturelle

RÉDACTEUR EN CHEF / EDITOR-IN-CHIEF: Jean-Philippe Siblet

ASSISTANTE DE RÉDACTION / ASSISTANT EDITOR: Sarah Figuet (naturae@mnhn.fr)

Mise EN PAGE / PAGE LAYOUT: Sarah Figuet

COMITÉ SCIENTIFIQUE / SCIENTIFIC BOARD:

Luc Abbadie (UPMC, Paris)

Luc Barbier (Parc naturel régional des caps et marais d'Opale, Colembert)

Aurélien Besnard (CEFE, Montpellier)

Vincent Boullet (Expert indépendant flore/végétation, Frugières-le-Pin)

Hervé Brustel (École d'ingénieurs de Purpan, Toulouse)

Patrick De Wever (MNHN, Paris)

Thierry Dutoit (UMR CNRS IMBE, Avignon)

Éric Feunteun (MNHN, Dinard)

Romain Garrouste (MNHN, Paris)

Grégoire Gautier (DRAAF Occitanie, Toulouse)

Olivier Gilg (Réserves naturelles de France, Dijon)

Frédéric Gosselin (Irstea, Nogent-sur-Vernisson)

Patrick Haffner (PatriNat, Paris)

Frédéric Hendoux (MNHN, Paris)

Xavier Houard (OPIE, Guyancourt)

Isabelle Le Viol (MNHN, Concarneau)

Francis Meunier (Conservatoire d'espaces naturels - Hauts-de-France, Amiens)

Serge Muller (MNHN, Paris)

Francis Olivereau (DREAL Centre, Orléans)

Laurent Poncet (PatriNat, Paris)

Nicolas Poulet (OFB, Vincennes)

Jean-Philippe Siblet (PatriNat, Paris)

Laurent Tillon (ONF, Paris)

Julien Touroult (PatriNat, Paris)

COUVERTURE / COVER:

Lestes macrostigma (Eversmann, 1836). Crédit photo: É. lorio.

Naturae est une revue en flux continu publiée par les Publications scientifiques du Muséum, Paris Naturae is a fast track journal published by the Museum Science Press, Paris

Les Publications scientifiques du Muséum publient aussi / The Museum Science Press also publish:

Adansonia, Zoosystema, Anthropozoologica, European Journal of Taxonomy, Geodiversitas, Cryptogamie sous-sections Algologie, Bryologie, Mycologie, Comptes Rendus Palevol.

Diffusion - Publications scientifiques Muséum national d'Histoire naturelle

CP 41 - 57 rue Cuvier F-75231 Paris cedex 05 (France)

Tél. : 33 (0)1 40794805 / Fax: 33 (0)1 40793840

diff.pub@mnhn.fr / https://sciencepress.mnhn.fr

(c) Publications scientifiques du Muséum national d'Histoire naturelle, Paris, 2022

ISSN (électronique / electronic): 1638-9387 


\title{
Les Arthropodes terrestres dans les études d'impact: limites actuelles et propositions pour une meilleure prise en compte des enjeux de conservation
}

\author{
Étienne IORIO \\ ECOTER (Écologie \& Territoires), 44 route de Montélimar, F-26110 Nyons (France) \\ etienne.iorio@ecoter.fr \\ François DUSOULIER \\ Muséum national d'Histoire naturelle, Direction générale déléguée aux collections, \\ 57 rue Cuvier, CP 43, F-75005 Paris (France) \\ francois.dusoulier@mnhn.fr
}

Fabien SOLDATI

Office national des Forêts, Laboratoire national d'Entomologie forestière, 2 rue Charles Péguy, F-11500 Quillan (France)

fabien.soldati@onf.fr

Franck NOËL

La motte, Saint-Martin-de-Connée, F-53160 Vimartin-sur-Orthe (France) noelfranck@yahoo.fr

Jean-Alain GUILLOTON

La Close des Saules, F-44810 Héric (France)

ja.guilloton@hotmail.fr

Guillaume DOUCET

8F rue Maurice Deslandres, F-21000 Dijon (France)

guillaume.jp.doucet@gmail.com

Philippe PONEL

Institut méditerranéen de Biodiversité et d'Écologie marine et continentale (IMBE), Aix-Marseille Université, Université d'Avignon, CNRS, IRD, Technopôle Arbois-Méditerranée,

Bât. Villemin, BP 80, F-13545 Aix-en-Provence cedex 04 (France) philippe.ponel@imbe.fr

Pascal DUPONT

PatriNat (OFB-CNRS-MNHN), Muséum national d'Histoire naturelle, 57 rue Cuvier, boîte postale 41, F-75231 Paris cedex 05 (France) pascal.dupont@mnhn.fr

Régis KRIEG-JACQUIER Opie-Odonates, BP30, 78041 Guyancourt cedex (France) regis.krieg.jacquier@gmail.com

Stéphane CHEMIN ECOTER (Écologie \& Territoires), 44 route de Montélimar, F-26110 Nyons (France) stephane.chemin@ecoter.fr 
Pierre TILLIER

Correspondant du Muséum national d'Histoire naturelle, 8 rue d'Aire, F-95660 Champagne-sur-Oise (France)

p.tillier.entomo@free.fr

Julien TOUROULT

PatriNat (OFB-CNRS-MNHN), Muséum national d'Histoire naturelle, 57 rue Cuvier, boîte postale 41, F-75231 Paris cedex 05 (France)

julien.touroult@ofb.gouv.fr

Soumis le 25 mars 2021 | Accepté le 14 juin 2021 | Publié le 23 février 2022

lorio É., Dusoulier F., Soldati F., Noël F., Guilloton J.-A., Doucet G., Ponel P., Dupont P., Krieg-Jacquier R., Chemin S. Tillier P. \& Touroult J. 2022. - Les Arthropodes terrestres dans les études d'impact: limites actuelles et propositions pour une meilleure prise en compte des enjeux de conservation. Naturae 2022 (4): 43-99. https://doi.org/10.5852/naturae2022a4

\section{RÉSUMÉ}

L'évaluation environnementale est un processus réglementaire visant à évaluer les projets de travaux ou d'aménagements qui peuvent avoir des incidences sur l'environnement. L'étude d'impact comporte un volet «milieux naturels» dans lequel un diagnostic écologique donne un état initial du site, notamment de ses enjeux faunistiques, floristiques et d'habitats, à partir de la bibliographie et de prospections dédiées. Il s'ensuit la proposition de mesures «ERC» (éviter, réduire, compenser) visant une neutralité écologique entre incidences et gains générés. Les Arthropodes constituent de loin le phylum le plus diversifié du règne animal, et devraient logiquement y tenir une place importante. Pourtant, en France, seuls quatre ordres comportent des espèces inscrites dans les textes réglementaires nationaux et européens. Le phylum des Arthropodes y est donc très peu représenté comparativement aux Vertébrés. Cet article questionne la place des Arthropodes dans les études d'impacts, leur utilité pour identifier des enjeux sur leurs habitats, et propose de nouveaux groupes qui pourraient apporter un complément à la fois taxinomique et écologique. L'examen d'un échantillon de 50 études d'impact montre que seuls trois principaux groupes y sont étudiés, tous comportant des espèces protégées: les papillons diurnes (Rhopalocères et Zygaena), les Odonates (Odonata) et les Orthoptères (Orthoptera); ainsi que quelques Coléoptères saproxylophages réglementés. Un effet "vase clos " probablement inhérent à la réglementation s'observe, car ces groupes sont ceux sur lesquels les connaissances progressent le plus et qui font l'objet de la plupart des évaluations de type Liste rouge. Ce déséquilibre entre les groupes abordés dans les études d'impact et la diversité réelle des Arthropodes continentaux, de leurs spécialisations et fonctions écologiques, de leurs particularités de répartition, induit que leurs enjeux sont évalués de manière inégale selon les habitats et secteurs géographiques concernés. Ainsi, les papillons diurnes et les Orthoptères mettent principalement en exergue des espèces et des enjeux de conservation pour les milieux prairiaux, puis secondairement les landes, fourrés et garrigues; les Odonates les milieux dulcicoles. Les Rhopalocères et Odonates comportent également un nombre non négligeable d'espèces à enjeux inféodées aux tourbières et marais. Ces groupes sont globalement de bons indicateurs des enjeux pour ces habitats. Les Coléoptères qui reviennent le plus régulièrement dans les études d'impact mettent surtout en valeur les arbres isolés, le bocage, les alignements d'arbres et les lisières forestières. A contrario, les milieux littoraux et les milieux forestiers fermés ne sont que très peu valorisés par ces groupes. Les papillons diurnes et Odonates sont parmi les Arthropodes avec les plus faibles taux d'endémisme en France métropolitaine, ce qui, de fait, sous-représente cet enjeu de conservation dans les études d'impacts. D’un point de vue fonctionnel, les coprophages, les nécrophages ou encore les prédateurs et décomposeurs litiéricoles sont aussi quasiment oubliés dans les études d'impacts. Cet article propose six groupes pour lesquels les connaissances ont bien progressé depuis 10 ou 20 ans et qui apporteraient un complément écologique et taxinomique aux espèces actuellement utilisées dans les études réglementaires: les Chilopodes (Chilopoda), les Isopodes terrestres (Isopoda, Oniscidea), les Coléoptères «Longicornes" (Cerambycidae et Vesperidae), Scarabaeoidea et Tenebrionidae, les Hétéroptères Pentatomoidea. La plupart des espèces à enjeux de conservation élevés de ces six groupes se situent sur les plages ou les dunes, les forêts ou les milieux tels que les grottes, les falaises ou les éboulis. Les Chilopodes, les Isopodes terrestres et les Coléoptères Tenebrionidae comportent un grand nombre d'endémiques induisant une responsabilité nationale élevée. Les plages et milieux côtiers, de la partie médiolittorale supérieure aux milieux dunaires, constituent l'exemple le plus frappant de sous-représentation au sein des espèces protégées et des groupes actuellement priorisés dans les études d'impact, comportant des dizaines d'espèces spécialisées à enjeux apparaissant menacées et aucun "parapluie» ne permettant de bien les préserver. De façon moindre, il en est de même pour les forêts fermées. Les grottes paraissent d'emblée moins concernées; cependant, 


\section{MOTS CLÉS \\ Arthropoda, évaluation environnementale, ERC, menaces, réglementation.}

la répartition souvent étroite des Arthropodes endémiques qu'elles abritent leur confere des enjeux à ne pas négliger. Les listes de protection paraissent donc à compléter par d'autres groupes que ceux pris en compte jusqu'ici, mais de manière appropriée aux Arthropodes continentaux: protection priorisant les habitats et non les spécimens, en corrélation avec les particularités de leur étude et de leur biologie. Pour les papillons diurnes, Odonates et Orthoptères, bien qu'un grand nombre d'espèces réglementées demeure pertinent, les listes paraissent à réajuster. La présente étude propose une liste argumentée de 135 espèces à forts enjeux de conservation dans neuf groupes d'Arthropodes. La prise en compte de l'ensemble par les bureaux d'études, les aménageurs et les autorités environnementales permettrait de refléter les enjeux des Arthropodes continentaux dans les études d'impact et donc de prendre des mesures plus adaptées à leur conservation.

\section{ABSTRACT \\ Terrestrial arthropods in impact studies: current limitations and proposals for better consideration of conservation issues.}

Environmental impact studies are a regulatory process to assess projects that may have significant environmental impacts. It includes a "natural environments" section, where an ecological diagnosis gives an initial status of the site to be assessed, including its wildlife, botanical and habitat concerns, based on bibliographical references and dedicated surveys. The result is the proposal for "ERC" measures (avoid, reduce, compensate) to preserve the environmental issues identified prior to the project.Arthropods are by far the most diverse phylum of the animal kingdom, and should therefore have an important place in an environmental impact study. However, only four orders have species listed in the current regulations, and the phylum of arthropods is poorly represented compared to vertebrates. The review of 50 impact studies shows that only three main groups are studied, relatively related to existing protections : butterflies (Zygaena included), dragonflies and Orthoptera, and some protected saprophagous wood-boring beetles. A 'closed-loop' effect occurs, likely inherent in the regulations and many of the associated actions, as these groups are the ones on which knowledge is most advanced and which are the subject of most Red List assessments. This imbalance between the groups covered in the impact studies and the actual diversity of continental arthropods, their specialisations and ecological functions, and their particular distributional characteristics, means that the issues at stake are assessed unequally depending on the habitats and geographical sectors concerned. Thus, butterflies and Orthoptera primarily highlight species and conservation issues for grassland environments, followed secondarily by moors, thickets and scrubland; dragonflies for freshwater environments. Butterflies and dragonflies also include a significant number of species of concern that are associated with peat bogs and marshes. These groups are generally good indicators of the issues affecting these habitats. The beetles that appear most regularly in impact studies highlight isolated trees, hedgerows, tree lines and forest edges. On the other hand, these groups are very few valuable to highlight conservation issues for arthropods of the coastal and closed forest environments. Butterflies and dragonflies are among the arthropods with the lowest rates of endemism in metropolitan France, which means that this conservation issue is under-represented in the impact studies. Functional guilds such as coprophagous, necrophagous or litter predators and decomposers are virtually almost forgotten in impact studies. This article proposes six groups for which knowledge has progressed well over the last 10 to 20 years and which would provide an ecological and taxonomic complement to the species currently used in regulatory studies: centipedes (Chilopoda), woodlice (Isopoda, Oniscidea), long-horned beetles (Cerambycidae and Vesperidae), Scarabaeoidea and Tenebrionidae, and shield-bugs (Heteroptera, Pentatomoidea). Most of the species of high concern in these six groups are found on beaches or dunes, forests or environments such as caves, cliffs or screes. Centipedes, woodlice and Tenebrionidae beetles have a large number of endemics and a high national responsibility. Beaches and coastal environments, from the shore to the dunes, are the most striking example of under-representation in protected species and current impact studies, with dozens of specialised species at risk and no umbrella species to protect them. To a lesser extent, the same applies to closed forests. Caves appear to be of less concern. However, the often narrow distribution of the endemic arthropods they house means that the stakes are not to be underestimated. The protection lists therefore need to be completed with groups other than those taken into account so far, but in a manner appropriate to continental arthropods. Protection should prioritise habitats and not specimens, in correlation with the particularities of their study and their biology. For butterflies, dragonflies and Orthoptera, although a large number of regulated species remain relevant, the lists need to be revised.This study proposes a list of 135 species with high conservation concerns in nine arthropod groups. If this list was taken into account by environmental agencies, landscape planners and environmental authorities, it would reflect the challenges for continental arthropods in impact studies and thus enable measures to be taken that are better suited to their conservation.
KEY WORDS Arthropoda environmenta assessment,

ERC,

threats, regulation. 


\section{INTRODUCTION}

Les projets qui, par leur nature, leur dimension ou leur localisation, sont susceptibles d'avoir des incidences notables sur l'environnement ou la santé humaine, font l'objet d'une évaluation environnementale en fonction de critères et de seuils définis par voie réglementaire et, pour certains d'entre eux, après un examen au cas par cas. Cette évaluation est un processus constitué de l'élaboration, par le maître d'ouvrage, d'une étude d'impact examinée ensuite par l'autorité compétente. Dans ce cadre, le porteur de projet doit analyser les incidences notables directes et indirectes d'un projet sur la biodiversité (Article L122-1 du Code de l'Environnement). Cette analyse suit des étapes aujourd'hui bien cadrées, que l'on appelle la séquence "ERC»: éviter, réduire, compenser (Article R122-5 du Code de l'Environnement).

La thématique biodiversité est l'une des plus denses de l'étude d'impact (thématique communément nommée « volet naturel de l'étude d'impact» ou VNEI). Si l'attention doit être portée en priorité aux espèces et aux habitats protégés, il n'en reste pas moins que c'est la biodiversité dans son ensemble, et en particulier les impacts du projet sur ces enjeux dans leur globalité, qui doit être analysée. Les statuts de protection sont fondés sur:

- l'Arrêté du 23 avril 2007 fixant les listes des Insectes protégés sur l'ensemble du territoire et les modalités de leur protection ("protection nationale»);

- les Directives Habitats-Faune-Flore (DHFF) 92/43/ CEE du 21 mai 1992 et 2009/147/ CE du 30 novembre 2009, ayant pour objectif de préserver la diversité biologique en Europe sous la forme d'un réseau de sites Natura 2000 , pour les habitats et espèces qui y sont désignées. Les projets d'aménagement susceptibles de porter atteinte à un tel site doivent faire l'objet d'un volet complémentaire d'analyse des incidences. Par ailleurs, l'Annexe 4 de la DHFF oblige à une protection stricte de certaines espèces, que l'on retrouve ainsi dans le point précédent;

- la Convention de Berne du 19 novembre 1979 (convention relative à la conservation des espèces sauvages et milieux naturels d'Europe), elle-même servant de base à la DHFF.

En France métropolitaine, un total de 123 espèces d'Arthropodes continentaux dispose d'un ou plusieurs statut(s) réglementaire(s), sans compter les espèces uniquement protégées en Île-de-France (Annexe 1). Cela ne représente qu'une infime proportion de ce phylum: moins de $0,3 \%$ de sa richesse spécifique connue dans notre pays, qui s'élève à environ 47000 espèces actuellement (Gargominy et al. 2020a). En comparaison, toutes les espèces autochtones de reptiles et Amphibiens, une large majorité des 578 espèces d'Oiseaux (https://www.oiseaux.net/oiseaux/famille.france. html, dernière consultation le 1er septembre 2020) et près de la moitié des 115 espèces de Mammifères continentaux (dont la totalité des Chiroptères) sont protégées (Arrêtés respectifs du 23 avril 2007 et du 29 octobre 2009 fixant les listes des Mammifères terrestres et des Oiseaux protégés sur l'ensemble du territoire et les modalités de leur protection; Fontaine et al. 2007; UICN et al. 2009). Les moyens alloués à l'étude et à la conservation des Arthropodes et des Vertébrés sont aussi très inégaux et largement en faveur des seconds, comme le soulignent Mammola et al. (2020) pour les espèces ciblées par le réseau Natura 2000. Un premier constat est donc que les Arthropodes sont très pauvrement représentés dans les textes réglementaires et demeurent négligés.

Un deuxième constat préliminaire est que ces 123 espèces appartiennent à seulement quatre ordres d'Insectes (Lépidoptères, Odonates, Orthoptères et Coléoptères), tandis que les Myriapodes Chilopodes et Diplopodes, les Arachnides et les Crustacés terrestres (Isopodes) sont totalement omis, à l'instar de tous les autres ordres d'Insectes présents en France (soit 22 sur 26). De même, les Listes rouges nationales et européennes, qui permettent de déterminer un statut de conservation, ne concernent quasiment toutes que les Lépidoptères Rhopalocères, les Odonates et les Orthoptères (Sardet \& Defaut 2004; Kalkman et al. 2010; Van Swaay et al. 2010; UICN France \& MNHN 2012; Hochkirch et al. 2016; UICN France et al. 2016), avec de rares exceptions telles que les Abeilles et les Coléoptères saproxyliques d'Europe, ou les Éphémères de France (Nieto et al. 2014; Cálix et al. 2018; UICN France et al. 2018); la situation étant similaire au regard des Listes rouges régionales. Nuançons toutefois avec le cas des Insectes protégés en Île-de-France (Arrêté du 22 juillet 1993 relatif à la liste des Insectes protégés en région Île-de-France, complétant la liste nationale), qui concerne des groupes d'Insectes plus diversifiés.

Au regard des espèces concernées par les statuts réglementaires, on peut s'interroger sur l'absence de représentation de certains habitats particuliers auxquels beaucoup d'Arthropodes sont strictement associés; e.g., des dizaines d'espèces halophiles ou psammophiles propres au littoral (Jaulin \& Soldati 2005; Iorio 2014; Séchet \& Noël 2015). Cette situation est d'autant plus critique que les littoraux méditerranéen et atlantique subissent une pression anthropique élevée - voire colossale pour le littoral méditerranéen - depuis plusieurs décennies. (Daligaux 2003; DREAL PACA 2013; Daeden 2015). Certaines guildes fonctionnelles largement reconnues sur le plan écologique sont très peu représentées, voire oubliées dans les textes de protection des espèces au niveau national et européen: coprophages, nécrophages, prédateurs et décomposeurs litiéricoles. Pourtant, certaines d'entre elles sont clairement en déclin (e.g., Numa et al. 2020).

Pour la prise en compte des espèces dans les politiques de conservation, on observe un cercle vicieux: les espèces peu étudiées sont insuffisamment prises en compte dans les Listes rouges, et in fine ne le sont pas non plus dans les listes de protection; ce qui, en retour, suscite moins de besoin de connaissance que pour les espèces inscrites dans les listes réglementaires (Cardoso et al. 2020). Aussi, on pouvait autrefois arguer que les autres groupes d'Arthropodes - en dehors des Rhopalocères et Zygènes, Odonates et Orthoptères - étaient insuffisamment connus au niveau de leur répartition, de leur écologie et/ou trop difficiles à aborder. Pourtant, depuis 10 à 20 ans, un essor des connaissances et du partage des données permet d'envisager de nouveaux groupes pertinents pour les démarches de conservation et en particulier pour les études d'impacts. 
TABLEAU 1. - Détail du premier niveau de la typologie EUNIS (Gayet et al. 2018).

Signification des codes du premier niveau de la typologie EUNIS

A - Habitats marins

B - Habitats côtiers

C - Eaux de surface continentales

D - Tourbières hautes et bas-marais

E - Prairies; terrains dominés par des herbacées non graminoïdes, des mousses ou des lichens

$\mathrm{F}$ - Landes, fourrés et toundras

G - Boisements, forêts et autres habitats boisés

$\mathrm{H}$ - Habitats continentaux sans végétation ou à végétation clairsemée

I - Habitats agricoles, horticoles et domestiques régulièrement ou récemment cultivés

$\mathrm{J}$ - Zones bâties, sites industriels et autres habitats artificiels

Comme nous le verrons plus loin, l'état des connaissances taxinomiques et écologiques concernant ces Arthropodes a connu un essor récent et considérable. Cette situation nous a amenés à nous poser diverses questions au sujet du volet naturel de l'étude d'impact, tel qu'il a été couramment abordé jusqu'ici; en particulier sur le diagnostic écologique servant d'état initial dans ce volet (i.e. le socle des mesures ERC). Parmi les plus cruciales, citons:

- les groupes d'Insectes actuellement évalués sont-ils appropriés à la mise en évidence correcte des enjeux de conservation des Arthropodes continentaux et de leurs habitats dans les études d'impact? Constituent-ils de bonnes espèces "parapluies» pour l'ensemble des Arthropodes continentaux à enjeux?

- quels sont la nature et le niveau des enjeux pour une sélection d'autres Arthropodes continentaux?

- les statuts des espèces actuellement protégées sont-ils tous pertinents et y a-t-il suffisamment d'espèces protégées parmi les Arthropodes continentaux?

- quels autres groupes pourraient être pertinents à intégrer dans ces études, tout en ne visant pas l'exhaustivité et en restant pragmatique?

À la suite de ces constats et pour tenter de répondre à ces questions, nous proposons de:

- faire un examen synthétique et critique du volet «Insectes et autres Arthropodes» sur un échantillon représentatif d'études d'impact;

- identifier les types d'habitats des espèces utilisées dans les études d'impact et identifier les lacunes;

- identifier les caractéristiques et enjeux de conservation des Arthropodes ciblés par les études d'impact actuellement;

- identifier les caractéristiques et enjeux de conservation d'autres groupes qui seraient de bons candidats pour ces études (avantages pratiques, autres spécialisations);

- confronter les évaluations actuelles avec les enjeux de tous ces groupes;

- proposer des perspectives d'évolution réalistes et des espèces d'Arthropodes candidates pour la séquence " $\mathrm{ERC}$ ».

\section{MATÉRIEL ET MÉTHODES}

Afin de faciliter la navigation dans l'article, une table des matières est disponible en page 99.

\section{EXAMEN DES ÉTUDES D'IMPACT}

Nous avons recherché des études d'impact librement accessibles sur internet, à l'aide du moteur de recherche Google. Nous avons utilisé quelques mots-clés comme notamment «évaluation environnementale», "diagnostic écologique», "volet naturel d'étude d'impact", les noms des divers départements et des grands types d'habitats pour trouver des études représentatives du territoire français et de ses particularités, réalisées dans la période 2010-2019. Dans la mesure du possible, nous avons privilégié les rapports centrés sur le diagnostic écologique ou le volet naturel de l'étude d'impact, plutôt que l'étude d'impact générale (cette dernière synthétisant parfois beaucoup les différentes parties, dont le volet naturel). Nous avons ainsi réuni une centaine d'études, dont 50 ont été tirées au hasard.

Sur ces 50 diagnostics écologiques et volets naturels d'études d'impact, nous avons synthétisé:

- la représentation des différents groupes d'Arthropodes continentaux sur l'ensemble des études;

- la proportion des niveaux d'enjeux de conservation par habitat d'après le premier niveau de la typologie EUNIS (Gayet et al. 2018) (Tableau 1), selon trois niveaux attribués pour les Arthropodes: nul/faible, modéré ou fort/majeur (une hiérarchisation avec les niveaux «nul», «très faible», "faible», «modéré» [ou «moyen»], «fort», «très fort» [ou «majeur»] s'observant dans les 50 rapports);

- la proportion moyenne des niveaux d'enjeux de conservation de la même manière que ci-dessus, pour le cumul des Vertébrés continentaux: Oiseaux, Mammiferes (dont Chiroptères), reptiles et Amphibiens;

- le taux d'occurrence d'espèces d'Arthropodes continentaux de niveau d'enjeu fort/majeur par habitat du premier niveau d'EUNIS;

- le taux d'occurrence d'espèces d'Arthropodes continentaux à statuts réglementaires par habitat du premier niveau d'EUNIS.

Quelques autres informations ont été relevées, comme par exemple le taux d'occurrence de certaines espèces protégées par rapport aux études concernant leur aire géographique (e.g., espèces protégées sténoméditerranéennes dans les études sur les régions méditerranéennes), afin de visualiser leur fréquence de manière plus fine. Précisons que les Insectes qui ne sont protégés qu'en Île-de-France ne seront pas abordés dans cet article, pour deux raisons: 
- la portée est trop limitée géographiquement par rapport au territoire métropolitain qui fait l'objet des analyses;

- aucune étude de notre tirage ne concerne la région francilienne.

La présentation des rapports est relativement hétérogène d'une étude à l'autre et a induit une adaptation dans leur exploitation. Par exemple, sur la base des inventaires menés par groupe faunistique, les enjeux sont parfois définis pour les espèces recensées et pour leurs habitats, appuyés par une cartographie pour les deux, facilitant ainsi grandement l'exercice. Dans d'autres cas, seuls les enjeux des espèces sont mis en exergue et non ceux des habitats d'espèces, ou alors par une unique synthèse tous groupes confondus par habitat. Pour ces seconds cas, nous avons pu rattacher les niveaux d'enjeux de chaque groupe aux principaux habitats en nous appuyant sur les exigences écologiques et/ou les habitats préférentiels des espèces détaillés dans les rapports. La simplicité du premier niveau d'EUNIS a facilité l'opération.

Par ailleurs, les habitats de chaque type du premier niveau d'EUNIS n'avaient aucune chance d'être représentés en nombre égaux, certains habitats étant nettement plus fréquents et étendus que d'autres sur le territoire et donc dans les études.

\section{Proposition D'UNE SÉLECTION DE GROUPES D'ARTHROPODES} CONTINENTAUX ET SYNTHÈSES DES CONNAISSANCES

Dans l'objectif de confronter les évaluations actuelles avec un plus large panel d'Arthropodes, une sélection de groupes à prendre en compte s'est faite de la manière suivante:

- groupes figurant parmi les trois les plus couramment abordés dans les études d'impact (i.e. les trois ordres les plus étudiés);

- groupes ayant un rang taxinomique de famille ou supérieur, encore peu ou non abordé dans ces études, mais présentant l'ensemble des caractéristiques écologiques et pratiques suivantes, répondant ainsi à la notion de groupe parapluie (Roberge \& Angelstam 2004) ou de groupe indicateur pour la conservation (Brown 1997):

- richesse spécifique modérée, entre 100 et 300 espèces en France métropolitaine;

- existence de clés d'identification récentes pour la majorité ou la totalité des espèces du groupe en France;

- exigences écologiques et répartition connues pour une majorité des espèces françaises;

- fonction écologique dans l'écosystème et capacités indicatrices de conditions de l'habitat (naturalité par exemple), de cortèges rares ou diversifiés, reconnues en Europe occidentale;

- accélération de la dynamique d'acquisition des connaissances au niveau national montrant une nette progression depuis 2010 ;

- existence de données partagées et accessibles (notamment dans le cadre du Système d'INformation de l'inventaire du Patrimoine naturel - SINP).

Comme il demeurait impossible d'aborder tous les groupes répondant aux critères ci-dessus, un dernier élément important de choix a été la complémentarité en termes d'exigences écologiques entre les groupes déjà étudiés dans les études d'impact et ceux proposés ici.
Pour chaque groupe retenu, une présentation synthétique a été rédigée pour retranscrire l'état des connaissances en France métropolitaine, avec des éléments sur les enjeux de conservation et sur les menaces principales à l'échelle du groupe taxinomique.

\section{SÉLECTION DES ESPÈCES À ENJEUX DE CONSERVATION}

Même si la connaissance est en nette progression sur l'ensemble des groupes étudiés, elle reste inégale et imparfaite pour la plupart d'entre eux. Pour effectuer une analyse homogène malgré les lacunes, nous avons arbitrairement fixé une limite de 15 espèces à enjeux de conservation de niveau fort ou majeur par groupe, soit 5 à $10 \%$ de leur richesse spécifique en France.

Pour sélectionner les espèces à forts enjeux de conservation dans chacun des groupes étudiés, nous avons pris en compte les références suivantes: ouvrages type Faune de France (e.g., Baraud 1992) et autres monographies similaires, atlas ou catalogues avec éléments sur la répartition, bases de données centralisées (INPN en particulier); références écologiques mettant en évidence les liens étroits avec des habitats et/ou des facteurs biotiques ou abiotiques particuliers; publications scientifiques faisant état de menaces avérées, d'une forte pression anthropique sur leurs habitats pour les espèces sténotopes ou sur leur territoire pour les espèces à répartition limitée. Lorsque des Listes rouges sont disponibles, le niveau des menaces repose en bonne partie sur la Liste rouge nationale ou européenne; mais aussi sur un examen des Listes rouges régionales ou encore le statut déterminant régional dans le programme ZNIEFF (informations centralisées au niveau de l'INPN).

Une matrice de critères, élaborée d'après les traits mis en avant par Roberge \& Angelstam (2004) pour des espèces "parapluies» et Le Berre et al. (2021) pour la hiérarchisation des espèces à prioriser pour la conservation, adaptée pour le contexte des études d'impacts, contribue à identifier les 15 espèces à plus forts enjeux dans chaque groupe (Annexe 2). L'avis d'expert affine ensuite la décision. Cette matrice est exposée dans le Tableau 2.

Pour un des groupes disposant d'une base de données centralisée des observations, les Chilopodes, nous avons aussi effectué une évaluation préliminaire des 15 espèces retenues avec la méthode de la Liste rouge au niveau national (UICN France 2018a), pour approfondir la régression des espèces. Ce fut aussi l'occasion de voir la faisabilité d'une Liste rouge pour un tel groupe. Nous avons utilisé les critères B1, B2 et D2 de la méthode Liste rouge pour les évaluations (UICN France 2018a). Les trois périodes retenues pour les comparaisons d'EOO (zone d'occurrence) et d'AOO (zone d'occupation) sont $<1935,1935-2009$ et 2010-2020. Ce travail a permis d'estimer leur catégorie de menaces au sein d'une éventuelle future Liste rouge en France métropolitaine. Cette catégorie de menace, bien qu'ici non officielle, a été déterminée avec soin en lien avec deux experts de la méthode Liste rouge de l'UMS PatriNat (OFB, CNRS, MNHN) et du comité français de l'UICN (Annexe 3).

Pour l'ensemble de cette troisième partie, les réflexions et les choix sur les espèces ont été faits en collaboration avec des spécialistes extérieurs. L'ensemble des participants est évoqué ci-dessous: 
TABLEAU 2. - Critères de choix pour les espèces à enjeux de conservation de niveau fort ou majeur. Un enjeu fort correspond à deux critères sans astérisques remplis (minimum); un enjeu majeur correspond quant à lui à un critère avec astérisque rempli ou quatre critères sans astérisque (minimum)

\begin{tabular}{|c|c|}
\hline ques pratiques: toutes requises & Caractéristiques biologiques \\
\hline $\begin{array}{l}\text { Pas d'ambiguïté taxinomique: } \\
\text { espèce ou sous-espèce formellement valide, } \\
\text { bien définie; } \\
\text { complexes d'espèces non retenus } \\
\text { Relativement simple à reconnaître: } \\
\text { caractères plutôt aisés à cerner; } \\
\text { peu d'espèces proches; } \\
\text { existence de clés/ouvrages; } \\
\text { identification possible sur le terrain avec } \\
\text { loupe à main, ou en laboratoire sur de } \\
\text { bonnes macrophotographies ou sous la loupe } \\
\text { binoculaire } \quad+ \\
\text { Relativement simple à détecter: } \\
\text { technique de recherche connue et aisée, } \\
\text { e.g., recherche et capture à vue dans les } \\
\text { habitats ou micro-habitats propices. }\end{array}$ & $\begin{array}{l}\text { Endémiques très localisées*: endémiques avec leurs deux stations les plus éloignées } \\
\text { distantes de moins de } 50 \text { km. } \\
\text { (Sub)endémiques plus larges: endémiques avec leurs deux stations les plus éloignées } \\
\text { distantes de plus de } 50 \text { km; couvrant de préférence deux pays au maximum si } \\
\text { subendémiques (e.g., France-Italie, France-Espagne). } \\
\text { Distribution relictuelle: e.g., boréo-alpine, forêts anciennes, etc. } \\
\text { Large répartition, mais très fragmentée: stations peu nombreuses et pour la plupart } \\
\text { distantes. } \\
\text { Espèce sténotope liée à un milieu rare et/ou localisé dans l'espace: e.g., plages } \\
\text { maritimes. } \\
\text { Espèce sténoèce liée à un micro-habitat rare, étroit et/ou à des conditions écologiques } \\
\text { particulières: e.g., cavités de très vieux arbres, face inférieure des galets de l'estran } \\
\text { en contact avec des sédiments plus fins, banquettes de posidonies, bois flottés, eaux } \\
\text { non polluées, plante-hôte rare, etc. } \\
\text { Faible capacité de dispersion: espèces documentées comme ayant une faculté de } \\
\text { dispersion inférieure à quelques kilomètres. } \\
\text { En déclin avéré ou déduit du déclin des habitats: au moins } 20 \% \text { des stations ou des } \\
\text { habitats favorables dégradés ou détruits et/ou soumis à forte anthropisation sur les } \\
100 \text { dernières années, et/ou menacés à court terme. } \\
\text { Si très fort déclin avéré ou déduit du très fort déclin des habitats*: disparition des } \\
\text { stations françaises connues > } 50 \text { \% et/ou réduction de l'aire d'occurrence > } 50 \% \\
\text { et/ou disparition des habitats propices avérée ou jugée > } 50 \% \text { sur } 100 \text { ans. }\end{array}$ \\
\hline
\end{tabular}

- Chilopodes: Étienne Iorio ; Jean-Jacques Geoffroy (MNHN), Guillaume Jacquemin et Antoine Racine (GRETIA) (spécialistes extérieurs);

- Isopodes terrestres: Franck Noël; Emmanuel Séchet (correspondant du MNHN) (spécialiste extérieur);

- Coléoptères: Fabien Soldati, Julien Touroult et Philippe Ponel; Thomas Barnouin (ONF), Hervé Brustel (École d'Ingénieurs de Purpan), Ludovic Fuchs (ONF), Nicolas Gouix (Conservatoire d'Espaces naturels [CEN] Occitanie), Arnaud Horellou (MNHN), Bruno Mériguet (Opie) et Stéphane Vassel (CPIE Pays Creusois) (spécialistes extérieurs);

- Hétéroptères Pentatomoidea: François Dusoulier; Thomas Cherpitel (Zicrona/GRETIA) (spécialiste extérieur);

- Lépidoptères diurnes: Jean-Alain Guilloton, Pascal Dupont, Étienne Iorio et François Dusoulier; Sylvain Delmas, Éric Drouet, Jean-Pierre Favretto (AER), Sonia Richaud (CEN PACA) et Marielle Tardy (Entomo\&Co) (spécialistes extérieurs);

- Odonates: Guillaume Doucet, Étienne Iorio, Régis KriegJacquier, François Dusoulier; Jean-Pierre Boudot (Université de Lorraine, Nancy) et Karim Guerbaa (CEN Limousin) (spécialistes extérieurs);

- Orthoptères: François Dusoulier; Yoan Braud (Entomia) (spécialiste extérieur);

- regard faunistique général: Pierre Tillier.

Afin de vérifier que les taxons proposés étaient bien complémentaires en termes d'écologie et de mise en évidence d'enjeux de conservation, nous avons ensuite calculé la part d'Arthropodes terrestres de niveau d'enjeu fort/majeur par grand type d'habitat (premier niveau d'EUNIS). Soulignons qu'il y a dans quelques cas une présence signalée dans deux types; e.g., pour certains halophiles surtout dans le type B
d'EUNIS, mais avec une occupation de la marge supérieure du type A. Quelques exemples d'habitats rencontrés lors de la synthèse inhérente à la première partie sur les études d'impact ont été réexaminés avec le prisme des groupes complémentaires étudiés dans cet article. L’objectif n'étant à ce stade que de sensibiliser, les renseignements précis sont volontairement omis. Seuls le département et le principal habitat sont précisés.

La taxonomie utilisée s'appuie sur le référentiel taxinomique national TaxRef, version 14.0 du 15 décembre 2020 (Gargominy et al. 2020b).

\section{RÉSULTATS}

\section{SYNTHÈSE DES 50 ÉTUDES D’IMPACT CONCERNANT}

LES ARTHROPODES CONTINENTAUX

Les études examinées concernent 38 départements français (Fig. 1) et comportent toutes un chapitre "Insectes", "Insectes et autres Arthropodes» ou «invertébrés». Deux groupes d'Arthropodes sont systématiquement étudiés dans les volets naturels d'études d'impact (Fig. 2): les papillons diurnes (Rhopalocères et Zygaenidae du genre Zygaena) et les Libellules (Odonata). Les Orthoptères (Orthoptera) sont pris en compte dans près des quatre cinquièmes de celles-ci. Une «entité réglementaire » apparaît ensuite avec les Coléoptères à enjeux réglementaires (e.g., Cerambyx cerdo Linnaeus, 1758 et Lucanus cervus (Linnaeus, 1758), étudiés dans $70 \%$ des cas, voire dans la quasi-totalité si l'on tient compte de leur potentialité de présence en fonction des habitats et du secteur géographique concernés. Au-delà de ces groupes, la prise en compte d'autres compartiments (ordre, sous-ordre ou famille) chute drastiquement, puisque la cinquième entité 


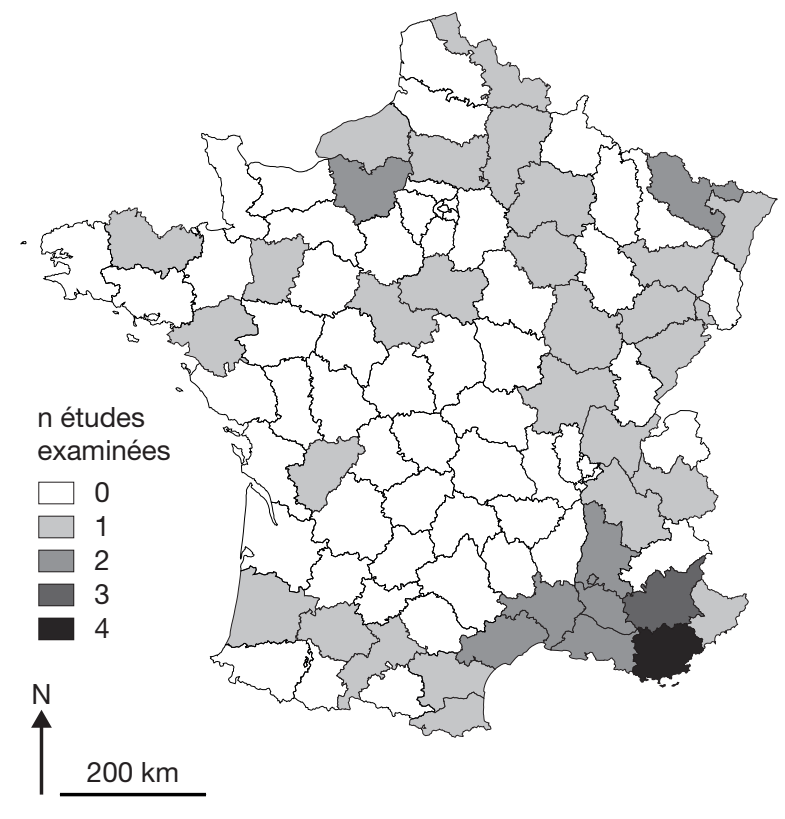

FIG. 1. - Répartition et nombres de volets naturels d'études d'impact examinés par département ( $\mathrm{n}$ total $=50 ;$ Annexe 4).

la plus fréquemment abordée - les Névroptères Ascalaphidae (dix espèces en France métropolitaine: Deliry \& Faton 2017) - n'apparaît que dans moins d'un cinquième des rapports.

Tous les autres groupes d'Arthropodes non inclus dans la Figure 2 sont (très) inférieurs à $10 \%$ ou, pour l'essentiel, ne sont pas abordés dans les études d'impact examinées.

Du côté des habitats, les "prairies et terrains dominés par des herbacées non graminoïdes, des mousses ou des lichens » (type E d'EUNIS), rassemblent les pourcentages les plus élevés pour les niveaux modéré et fort/majeur d'enjeux de conservation des Insectes et autres Arthropodes (Fig. 3). 62,2 \% des habitats prairiaux hébergent des Arthropodes d'enjeu modéré ou supérieur; un tiers des enjeux de niveau fort ou supérieur. L'addition des pourcentages des niveaux modéré et fort/majeur chute à 40,6\% pour le deuxième habitat à plus forte proportion d'enjeux notables ( $\mathrm{C}$ : « eaux de surface continentales", qui incluent notamment les milieux dulcicoles lotiques et lentiques "naturels ", avec ou sans végétation émergée) et à 18,8\% pour le seul niveau fort/majeur. Ces taux décroissent encore nettement pour les autres types; le type G ("boisements, forêts et autres habitats boisés») ne se trouvant qu'à respectivement $22,7 \%$ et $11,4 \%$. Aucun Arthropode de niveau modéré ou supérieur n'a été recensé dans les types A («habitats marins», qui incluent aussi la zone médiolittorale supérieure de l'estran, ainsi que par exemple le schorre) et B ("habitats côtiers", qui concernent entre autres les dunes côtières et les hauts de plages au-dessus de la laisse de mer). Peu d'enjeux modérés et aucun enjeu fort/majeur n'ont été définis dans les types $\mathrm{H}$ ("habitats continentaux sans végétation ou à végétation clairsemée", qui comprennent entre autres les éboulis et les grottes), I ("habitats agricoles, horticoles et domestiques régulièrement ou récemment cultivés») et J "zones bâties, sites industriels et autres habitats artificiels ") ; les deux derniers correspondant à des habitats (très) anthropisés.

La moyenne des mêmes analyses sur les mêmes études d'impact pour les Vertébrés terrestres (Oiseaux, Mammiferes [dont Chiroptères], reptiles-Amphibiens) donne des pourcentages sensiblement différents pour les niveaux d'enjeux par habitat (Fig. 4). Il n'y a pas de nette dominance d'un type d'habitat par rapport aux autres. Une analyse similaire à celle effectuée pour les Arthropodes montre que quatre types (D [tourbières, marais], E [prairies], F [landes, fourrés, garrigues] et $\mathrm{G}$ [forêts]) diffèrent peu entre eux, entre 0 et $5 \%$ pour les niveaux modéré et fort/majeur cumulés. Le type $\mathrm{G}$ se situe très légèrement devant les autres, avec $52,7 \%$, dont 19,9 \% pour le seul niveau fort/majeur; le type E étant à respectivement $47,7 \%$ et $19,3 \%$. On constate en revanche un taux d'enjeux de niveau modéré à fort/majeur moins élevé pour les types I (habitats agricoles, horticoles) et J (zones bâties), et très peu élevé pour le type A (habitats marins).

Si l'on décompose les groupes de Vertébrés pour les prairies (E), aucun taxon n'atteint le pourcentage des niveaux modéré et fort cumulés chez les Arthropodes. Celui des reptiles-Amphibiens, pris ici comme une seule entité (car généralement étudié par le même expert) en est le plus proche, avec 53,3 \% d'enjeux de niveau modéré à fort/majeur. Concernant les forêts $(G)$, tous les groupes présentent davantage d'enjeux modérés à forts que les Arthropodes: $34,1 \%$ chez les reptiles-Amphibiens, $61,4 \%$ chez les Oiseaux et $62,8 \%$ chez les Mammifères.

Le type $\mathrm{B}$ (habitats côtiers) présente quant à lui nettement plus d'enjeux remarquables chez les Vertébrés (Fig. 4) que chez les Arthropodes (Fig. 3) d'après les études examinées (de 20 à $60 \%$ d'enjeux de niveau modéré et fort/majeur cumulés selon les groupes). Il en est de même pour le type $\mathrm{H}$ (habitats continentaux sans végétation, dont grottes) (de 25 à $50 \%$ ).

Les taux d'occurrences des espèces d'Arthropodes continentaux à enjeux de conservation de niveau fort/majeur ou à statuts réglementaires présentent d'importantes différences en fonction des habitats (Fig. 5). On constate de nouveau une forte dominance pour les prairies $(\mathrm{E})$, que ce soit pour les espèces de niveau d'enjeu fort ou supérieur, ou pour les espèces à statuts réglementaires. Malgré la représentation presque identique des milieux prairiaux (E) et forestiers $(\mathrm{G})$ dans les études examinées (n respectifs $=45$ et 44 ), le pourcentage d'espèces à enjeu fort/majeur est quasiment sept fois supérieur dans le premier par rapport au second; 3,8 fois supérieur pour les espèces protégées. Les habitats $\mathrm{A}, \mathrm{B}, \mathrm{H}$ et J (n cumulé $=50$ ) n'ont montré aucune espèce d'Arthropode à enjeu fort/majeur ni aucune à statut réglementaire.

Quasiment toutes les espèces définies comme possédant des enjeux remarquables (forts/majeurs ou protégés) sont des Lépidoptères diurnes, Odonates et Orthoptères; ou deux Coléoptères réglementés, Cerambyx cerdo et Lucanus cervus. Plus rarement, trois autres espèces protégées, le Coléoptère Rosalia alpina (Linnaeus, 1758) et les deux Hétérocères Eriogaster catax (Linnaeus, 1758) et Proserpinus proserpina (Pallas, 1772) sont aussi mis en exergue. Enfin, exceptionnellement, des enjeux forts sont signalés dans de très rares autres groupes, en particulier les névroptères (mais aucun n'est réglementé). 


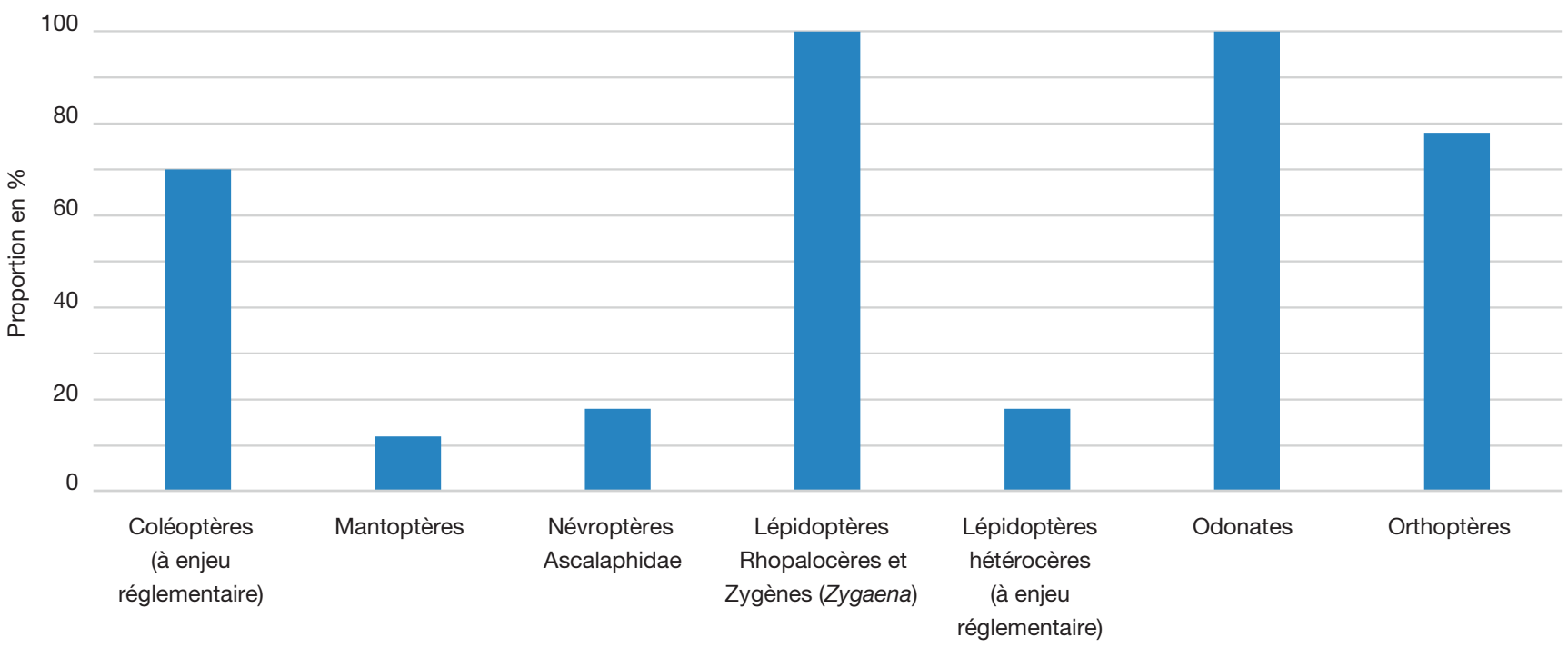

FIG. 2. - Proportion des groupes d'Arthropodes les plus étudiés (> $10 \%$ ) sur 50 volets naturels d'études d'impact.

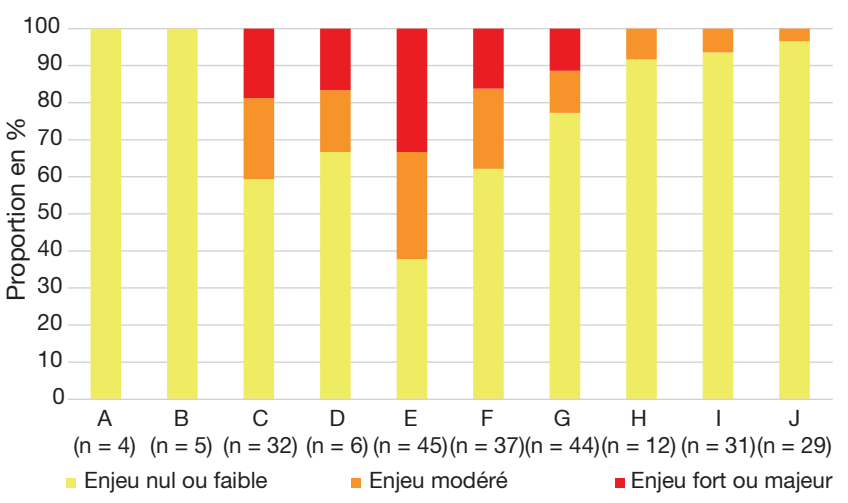

FIG. 3. - Proportion des enjeux des Arthropodes continentaux par grand type d'habitat EUNIS relevés à partir de 50 volets naturels d'études d'impact ( $\mathrm{n}=$ nombre d'études où l'habitat apparaît). A, habitats marins; $\mathbf{B}$, habitats côtiers; $\mathbf{C}$, eaux de surface continentales; $\mathbf{D}$, tourbières hautes et bas-marais ; E, prairies, terrains dominés par des herbacées non graminoïdes, des mousses ou des lichens; F, landes, fourrés et toundras; G, boisements, forêts et autres habitats boisés; $\mathbf{H}$, habitats continentaux sans végétation ou à végétation clairsemée; I, habitats agricoles, horticoles et domestiques régulièrement ou récemment cultivés; $\mathbf{J}$, zones bâties, sites industriels et autres habitats artificiels.

Certaines espèces protégées reviennent plus ou moins fréquemment dans les études: le Papilionidae Zerynthia polyxena (Denis \& Schiffermuller, 1775) est pris en considération dans plus de la moitié $(53,8 \%)$ des études concernant son aire géographique $(\mathrm{n}=13)$ et dans $14 \%$ des 50 études d'impact. Les espèces trouvées appuyant la définition d'enjeux forts ou réglementaires pour les habitats forestiers sont presque toujours Cerambyx cerdo et Lucanus cervus, plus rarement Rosalia alpina.

\section{SYNTHÈSE DES CONNAISSANCES BIOGÉOGRAPHIQUES,} ÉCOLOGIQUES ET DE LA VULNÉRABILITÉ GLOBALE POUR UNE SÉLECTION D'ARTHROPODES CONTINENTAUX Neuf groupes d'Arthropodes terrestres ont été sélectionnés sur la base des critères écologiques et pratiques définis, afin de procéder à un examen des enjeux de conservation et de connaissance: les trois déjà classiquement abordés dans les études d'impact, à

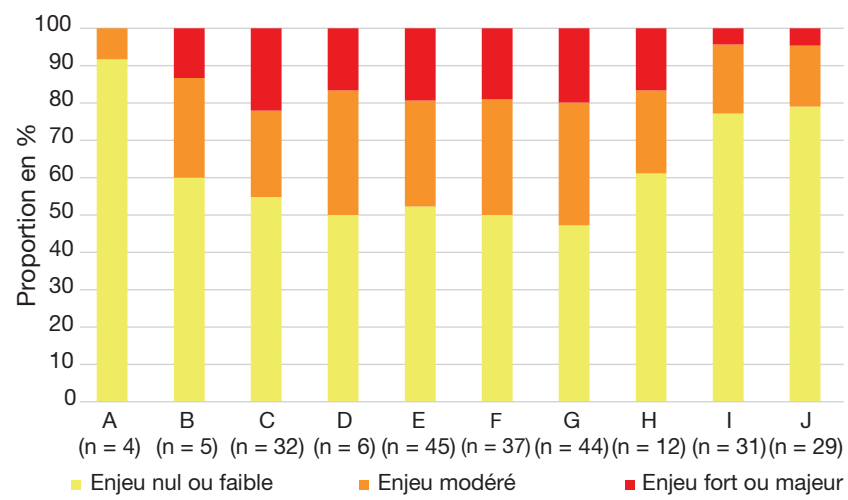

FIG. 4. - Proportions d'enjeux des Vertébrés continentaux par grand type d'habitat EUNIS sur 50 volets naturels d'études d'impact ( $n=$ nombre d'études où l'habitat apparaît). $\mathbf{A}$, habitats marins; $\mathbf{B}$, habitats côtiers; $\mathbf{C}$, eaux de surface continentales; $\mathbf{D}$, tourbières hautes et bas-marais; $\mathbf{E}$, prairies, terrains dominés par des herbacées non graminoïdes, des mousses ou des lichens; $\mathbf{F}$, landes, fourrés et toundras; G, boisements, forêts et autres habitats boisés; $\mathbf{H}$, habitats continentaux sans végétation ou à végétation clairsemée; I, habitats agricoles, horticoles et domestiques régulièrement ou récemment cultivés; $\mathbf{J}$, zones bâties, sites industriels et autres habitats artificiels.

savoir les Lépidoptères diurnes (Rhopalocères et Zygaena), les Odonates (Odonata) et les Orthoptères (Orthoptera); six autres avec des exigences écologiques complémentaires aux premiers. Il s'agit des Chilopodes (Chilopoda), une des quatre classes de Myriapodes; des Cloportes (Isopoda, Oniscidea), groupe de Crustacés exclusivement terrestre; et de quatre groupes d'Insectes, les Coléoptères «Longicornes» (Cerambycidae et Vesperidae), les Coléoptères Scarabaeoidea et Tenebrionidae, et les Hétéroptères Pentatomoidea. Initialement, nous envisagions d'intégrer les Diptères Syrphidae et Tipulidae, intéressants sur le plan écologique. Cependant, pour les premiers, leur trop grande diversité (563 espèces [Vanappelghem et al. 2020]) nous a conduits à les écarter de ce travail. Pour les Tipulidae, la richesse spécifique conviendrait avec 177 espèces (P. Tillier comm. pers.), mais les connaissances s'avèrent encore insuffisantes (10000 données en janvier 2021, soit 56,5 données/espèce en moyenne). 


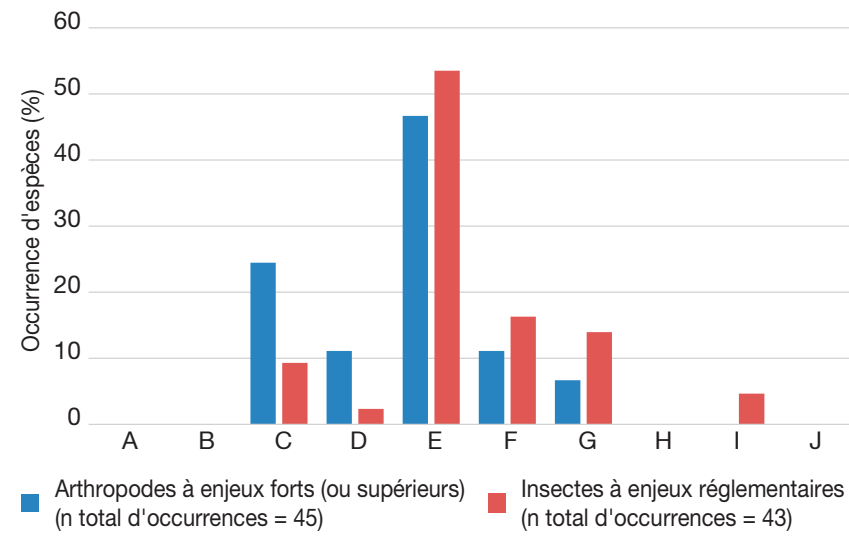

FIG. 5. - Pourcentages des occurrences d'Arthropodes continentaux à enjeux forts/majeurs ou à statuts réglementaires par grand type d'habitat EUNIS sur 50 études d'impact. A, habitats marins; B, habitats côtiers; C, eaux de surface continentales; $\mathbf{D}$, tourbières hautes et bas-marais; $\mathbf{E}$, prairies, terrains dominés par des herbacées non graminoïdes, des mousses ou des lichens; $\mathbf{F}$, landes, fourrés et toundras; $\mathbf{G}$, boisements, forêts et autres habitats boisés; $\mathbf{H}$, habitats continentaux sans végétation ou à végétation clairsemée; $\mathbf{I}$, habitats agricoles, horticoles et domestiques régulièrement ou récemment cultivés; J, zones bâties, sites industriels et autres habitats artificiels.

Les Abeilles sauvages (Hyménoptères Apoidea) sont largement en dehors de nos critères de sélection, avec une richesse spécifique supérieure à 900 espèces en France et une difficulté d'identification élevée pour une majorité des espèces (Le Féon et al. 2016; Le Divelec 2021). Ceci est illustré par la Liste rouge européenne des Abeilles, qui comporte plus de $55 \%$ des espèces classées en "données insuffisantes» (DD) (Nieto et al. 2014). D'autres groupes auraient pu être candidats, comme certaines familles abordables de macro-hétérocères, les grands Carabes (Coléoptères Carabinae), les Coléoptères nécrophages (Silphidae), d'autres Coléoptères saproxyliques, certaines familles d'Araignées peu ou modérément riches en espèces (une Liste rouge nationale étant d'ailleurs en cours de réalisation sur les Araignées), ou encore les Éphémères. Cependant, ils n'ont pas pu être exploités dans le cadre limité de cette analyse, qui ne se veut pas exhaustive.

\section{Chilopoda}

Les Chilopodes (Fig. 6) constituent la deuxième classe de Myriapodes ou "mille-pattes» en termes de diversité, avec 3325 espèces dans le monde, 550 en Europe et 149 en France (Iorio 2014; Simaiakis \& Strona 2014; Bonato et al. 2016), et celle dont la connaissance a le plus progressé dans notre pays. Fin 2020, environ 27000 données ont été réunies en France sur plus de 70000 individus identifiés à l'espèce (bases MyriaFrance et GRETIA), soit une moyenne de 181 données/espèce (Tableau 3). $85 \%$ de ces données ont été produites depuis 2000. Cette augmentation notable des connaissances est due à deux facteurs: d'une part, à la parution de clés pratiques rendant l'identification très abordable (Iorio \& Geoffroy 2008; Iorio 2010a, b; Iorio \& Labroche 2015); d'autre part, à une activité de plus en plus importante depuis 2010, couronnée par la création du groupe "Myria-France» en 2018 réunissant une quarantaine d'invertébristes. D'autres naturalistes ont été formés pour élaborer un atlas régional en cours via le

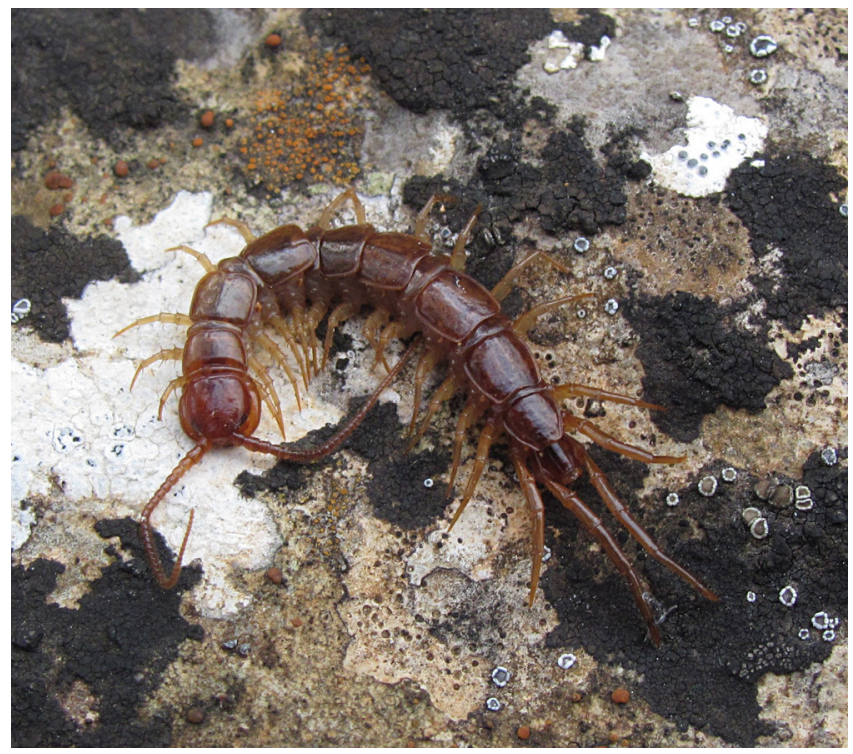

FIG. 6. - Exemple d'un chilopode: Lithobius pyrenaicus Meinert, 1872. Crédit photo: É. lorio.

GRETIA (Racine \& Iorio 2020). En plus de la dynamique bénévole croissante, des missions d'inventaires sont sur le point de s'achever, tandis que d'autres sont en cours. Leurs données se cumuleront d'ici peu à celles de Myria-France.

$40 \%$ des espèces françaises sont endémiques ou subendémiques (Iorio 2014). Bien que quelques (sub)endémiques soient relativement bien distribués sur le territoire, comme Arctogeophilus inopinatus (Ribaut, 1910) que l'on peut trouver du Massif armoricain au centre de la France, plus de la moitié présente une répartition restreinte (Tableau 4). Certains endémiques français sont extrêmement localisés, ce qui leur confère une grande sensibilité: deux exemples parmi d'autres sont Geophilus persephones Foddai \& Minelli, 1999, troglobionte du gouffre de la Pierre Saint-Martin (64) et Clinopodes vesubiensis Bonato, Iorio \& Minelli, 2011, des montagnes de la Vésubie (06).

Les Chilopodes sont des prédateurs inaptes au vol, vivant au sol, sous les pierres, les bois morts et dans la litière. Ils sont discrets car lucifuges, mais assez faciles à rechercher à vue sous les repaires favorables et à l'aide de tamisages de sol et de litière. Ils peuvent avoir des densités importantes dans les forêts, jusqu’à plus de 350 spécimens $/ \mathrm{m}^{2}$ en France et plus de 480 spécimens/ $\mathrm{m}^{2}$ dans les vieilles forêts de Slovaquie (Jabin 2008; Iorio \& Pétillon 2020). Ils jouent un rôle important dans la régulation des décomposeurs (Menta \& Remelli 2020). Ils sont considérés comme de bons bioindicateurs de la faune du sol et de la litière, car ils sont sensibles à de nombreuses variables, dont le $\mathrm{pH}$, l'humidité, la température et la structure du micro-habitat (Jabin 2008; Voigtländer 2011 ; Menta \& Remelli 2020). La plupart des espèces sténotopes se localisent dans les forêts, les plages et les grottes (Iorio 2014). L'habitat de certains halophiles est très étroit, e.g., Schendyla monodi (Brolemann, 1924) qui ne vit que dans le schorre et la haute slikke sur la façade atlantique, ou le méditerranéen Tuoba poseidonis (Verhoeff, 1901) dont le milieu n'excède pas trois mètres de large sur la plage (Racine $\&$ Iorio 2017; Iorio et al. 2020). 
TABLEAU 3. - Informations sur la richesse spécifique, les données existantes et l'endémisme en France fin 2020, ainsi que les Listes rouges des groupes étudiés. Abréviation: AURA, Auvergne-Rhône-Alpes; end, endémique(s); LRE, Liste rouge européenne; LRN, Liste rouge nationale; LRR, Liste rouge régionale (uniquement les listes labellisées UICN; découpage < 2016); N, nombre; $\mathbf{N}$ données, nombre de données accessibles, source INPN ou base(s) rassemblant actuellement l'essentiel de la dynamique d'acquisition des connaissances (requête effectuée début janvier 2021), ou chiffre issu d'une estimation des auteurs; subend, subendémique(s). Ces chiffres en constante évolution pour les neuf groupes ne se veulent pas exhaustifs mais seulement indicatifs. *, proposition d'évaluation pour 15 espèces (Annexe 3); **, $66 \%$ d'endémiques/subendémiques avec sous-espèces (300 taxons terminaux).

\begin{tabular}{|c|c|c|c|c|c|c|c|c|}
\hline Groupe & $\begin{array}{l}\mathrm{N} \text { espèces } \\
\text { (FR) }\end{array}$ & $\mathrm{N}$ données & $\begin{array}{l}\mathrm{N} \text { moyen } \\
\text { données/ } \\
\text { espèce }\end{array}$ & $\begin{array}{l}N \text { end } \\
\text { et } \\
\text { subend }\end{array}$ & $\begin{array}{l}\% \text { end } \\
\text { et subend }\end{array}$ & $\begin{array}{l}\text { LRN } \\
\text { et LRE }\end{array}$ & LRR & Remarques \\
\hline Chilopoda & 149 & 27000 & 181 & 60 & $40,3 \%$ & Non* & Non & $\begin{array}{l}\text { N données: bases Myria-France et } \\
\text { GRETIA (A. Racine comm. pers.) }\end{array}$ \\
\hline Oniscidea & 220 & 33000 & 150 & 62 & $28,2 \%$ ** & Non & Non & $\mathrm{N}$ données: base INPN \\
\hline «Longicornes» & 242 & 150600 & 622 & 6 & $2,5 \%$ & Oui (LRE) & 2 & $\begin{array}{l}\text { N données: base INPN } \\
\text { LRR }=\text { AURA et Limousin }\end{array}$ \\
\hline Scarabaeoidea & 300 & 125000 & 416 & 17 & $5,7 \%$ & Oui (LRE) & Non & $\begin{array}{l}\text { N données: base INPN } \\
\text { Existence d'une LR } \\
\text { méditerranéenne }\end{array}$ \\
\hline Tenebrionidae & 219 & 50000 & 228 & 67 & $30,6 \%$ & Oui (LRE) & 1 & $\begin{array}{l}\text { N données: base F. Soldati (faune } \\
\text { de France en prép.) } \\
\text { LRR = AURA }\end{array}$ \\
\hline Pentatomoidea & 154 & 135000 & 876 & 4 & $2,6 \%$ & Non & Non & $\begin{array}{l}\text { N données: base INPN et } \\
\text { estimation F. Dusoulier }\end{array}$ \\
\hline $\begin{array}{l}\text { "Rhopalocères» } \\
\text { et Zygaena }\end{array}$ & 300 & $>4000000$ & $>13300$ & 18 & $6,0 \%$ & Oui & 15 & $\begin{array}{l}\mathrm{N} \text { données, références par région: } \\
\text { Annexe } 5 \text {. }\end{array}$ \\
\hline Odonata & 98 & $>1000000$ & $>10200$ & $0(5)$ & $\begin{array}{l}0 \% \\
(5,1 \%)\end{array}$ & Oui & 15 & $\begin{array}{l}\mathrm{N} \text { données: base INPN. } \\
\mathrm{N} \text { subend (5) = en élargissant } \\
\text { la notion (e.g. espèces ibéro- } \\
\text { françaises). }\end{array}$ \\
\hline Orthoptera & 232 & $>1030000$ & $>4400$ & 47 & $20,2 \%$ & Oui (LRE) & 9 & $\begin{array}{l}\mathrm{N} \text { données, références par région: } \\
\text { Annexe } 6 .\end{array}$ \\
\hline
\end{tabular}

TABLEAU 4. - Étendue de la répartition des endémiques et subendémiques français pour cinq groupes d'Arthropodes terrestres. *, à vol d'oiseau.

\begin{tabular}{|c|c|c|c|c|c|c|}
\hline \multirow[b]{2}{*}{ Groupe } & \multicolumn{2}{|c|}{$\begin{array}{l}\text { Endémiques ou subendémiques à } \\
\text { large répartition (= deux stations } \\
\text { les plus distantes }>150 \mathrm{~km}^{\star} \text { ) }\end{array}$} & \multicolumn{2}{|c|}{$\begin{array}{l}\text { Endémiques ou subendémiques à } \\
\text { répartition étroite (= deux } \\
\text { stations les plus distantes }>50 \mathrm{~km} \\
\text { et } \leq 150 \mathrm{~km}^{\star} \text { ) }\end{array}$} & \multicolumn{2}{|c|}{$\begin{array}{l}\text { Endémiques ou subendémiques très } \\
\text { localisés (= deux stations les plus } \\
\text { distantes } \leq 50 \mathrm{~km}^{\star} \text { ) }\end{array}$} \\
\hline & $\begin{array}{l}\text { Nombre } \\
\text { d'espèces } \\
\text { concernées }\end{array}$ & $\begin{array}{l}\text { \% richesse } \\
\text { spécifique } \\
\text { nationale }\end{array}$ & $\begin{array}{l}\text { Nombre } \\
\text { d'espèces } \\
\text { concernées }\end{array}$ & $\begin{array}{l}\text { \% richesse } \\
\text { spécifique } \\
\text { nationale }\end{array}$ & $\begin{array}{l}\text { Nombre } \\
\text { d'espèces } \\
\text { concernées }\end{array}$ & $\begin{array}{l}\text { \% richesse } \\
\text { spécifique } \\
\text { nationale }\end{array}$ \\
\hline $\begin{array}{l}\text { Lépidoptères } \\
\text { Rhopalocères / } \\
\text { Zygaena }\end{array}$ & 18 & $6 \%$ & 0 & $0 \%$ & 0 & $0 \%$ \\
\hline Chilopodes & 29 & $19,5 \%$ & 17 & $11,4 \%$ & 14 & $9,4 \%$ \\
\hline $\begin{array}{l}\text { Coléoptères } \\
\text { Tenebrionidae }\end{array}$ & 46 & $21 \%$ & 11 & $5 \%$ & 10 & $4,6 \%$ \\
\hline $\begin{array}{l}\text { Hétéroptères } \\
\text { Pentatomoidea }\end{array}$ & 4 & $2,6 \%$ & 0 & $0 \%$ & 0 & $0 \%$ \\
\hline Orthoptères & 37 & $15,9 \%$ & 3 & $1,3 \%$ & 7 & $3 \%$ \\
\hline
\end{tabular}

La situation des halophiles méditerranéens est très préoccupante. Très peu mobiles, ils ont disparu de la majeure partie des côtes françaises en raison de la pression anthropique sur leurs habitats exigus, et ne subsistent plus que dans des secteurs restreints encore préservés (Iorio et al. 2015, 2020; Geoffroy \& Iorio 2019). Plusieurs espèces inféodées aux boisements caducifoliés froids et humides (Iorio 2014; Iorio \& Pétillon 2020) sont jugées menacées à moyen ou long terme, a fortiori avec le réchauffement climatique; surtout celles liées aux hêtraies (Piedallu et al. 2009; UICN France 2016). La recolonisation de sites forestiers perturbés puis restaurés est très longue, voire inexistante pour les espèces sylvicoles exigeantes (Dunger \&
Voigtländer 2009), probablement en partie à cause de leurs faibles capacités de dispersion (Grgič \& Kos 2005). Paraissant moins concerné, le milieu souterrain n'est pas exempt d'impact (Karam-Gemael et al. 2018; Borges et al. 2019). Ainsi, deux des huit stations de l'endémique varois Lithobius fagniezi Ribaut, 1926 (grotte des Fées à Hyères; réseau souterrain à Mazaugues) ont disparu à cause de l'anthropisation : lotissements, carrières, inondation (France 3 PACA 2019; P. Courbon [CDS83] comm. pers.; É. Iorio \& P. Ponel comm. pers.). En plus de la destruction directe des habitats, d'autres actions ont un effet négatif sur les Chilopodes: éclaircies forestières, plantation de conifères allochtones, suppression 


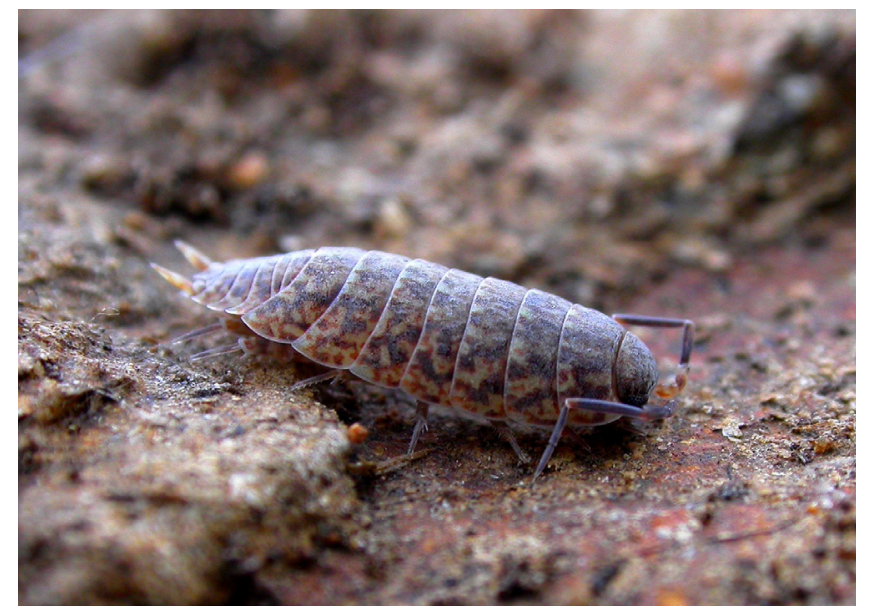

FIG. 7. - Exemple d'un isopode: Caeroplastes porphyrivagus Verhoeff, 1933. Crédit photo: F. Noël.

des abris naturels (e.g., repaires pierreux et laisses de mer sur les plages), surpiétinement, pollution des sols (Poser 1990; Voigtländer 2011 ; Iorio 2014). Les Chilopodes ne bénéficient d'aucune Liste rouge en France. Pourtant, l'amélioration des connaissances offre la possibilité de proposer une telle liste dans notre pays (Annexe 3).

\section{Isopoda Oniscidea}

Les Crustacés Isopodes (Fig. 7) constituent un groupe diversifié avec plus de 11000 espèces terrestres ou aquatiques décrites dans le monde. Les Oniscidea (Crustacés Isopodes terrestres) représentent 3700 espèces décrites (Sfenthourakis \& Taiti 2015) dont 220 sont présentes en France (Séchet \& Noël 2015). Le degré de connaissance sur ce groupe est correct, mais très hétérogène selon les régions. Débuté en 2011, l'Inventaire permanent des Crustacés terrestres de France métropolitaine (INPN, MNHN Paris) comporte à présent près de 33000 données documentant l'ensemble des taxons terminaux consécutivement à la saisie des données bibliographiques anciennes; soit une moyenne de 150 données/espèce (Tableau 3). Un regain de prospection est observé à partir des années 2000, après presque 30 années d'essoufflement et de disparition progressive des taxonomistes français s'intéressant à ce groupe. De nouvelles espèces restent à décrire comme le prouvent les travaux récents de Dalens sur les Oritoniscus pyrénéens (Dalens et al. 1996, 1997; Dalens 1998), ou ceux de Taiti \& Ferrara (1996) concernant la faune insulaire de Corse.

$28 \%$ des espèces sont endémiques ou subendémiques de France. Mais en comptant les nombreuses sous-espèces, on relève $45 \%$ d'endémiques et $66 \%$ d'endémiques et subendémiques sur les 300 taxons terminaux répertoriés en France métropolitaine ( $=$ taxons connus d'un autre territoire limitrophe: franco-espagnols, corso-sardes, du Pays Basque, etc.). L'essentiel de l'endémisme est localisé dans les Pyrénées, le sud-est de la France ou encore la Corse (Séchet \& Noël 2015). Plusieurs de ces (sous-)espèces ne sont décrites que d'une seule grotte ou de seulement quelques localités et nombre d'entre elles n'ont plus été signalées depuis plusieurs dizaines d'années.
Les Isopodes terrestres ont colonisé la quasi-totalité des biotopes, à l'exception des plus hautes altitudes. Certaines espèces qui s'étaient affranchies de l'élément aquatique, se sont réadaptées pour devenir amphibies telles que les Ligia ou certaines espèces de Tylos (Brown \& Odendaal 1994). Les Isopodes terrestres sont principalement des décomposeurs (phytosaprophages), qui jouent un rôle actif dans la décomposition de la litière (David 2014; Špaldoňová \& Frouz 2014; Joly et al. 2020) ou des laisses de mer (Zimmer et al. 2002 ; De la Huz et al. 2006) et peuvent atteindre localement des densités remarquables (Dias et al. 2005). La distribution et l'abondance des Isopodes terrestres sont dictées par des facteurs biotiques, notamment la ressource en matière organique décomposable, mais également par des facteurs abiotiques, qui régissent la distribution des espèces les plus sténotopes: nombre d'endémiques sont inféodés à des milieux aux conditions d'hygrométrie et de température stables, telles que les grottes ou le milieu souterrain superficiel (MSS).

Certains Cloportes halophiles apparaissent clairement menacés, comme notamment Trichoniscus halophilus Vandel, 1951, méditerranéen insulaire en France, ou encore Armadillidium album Dollfus, 1887, surtout présent sur le littoral de l'Atlantique et de la Manche. Ceux-ci vivent généralement dans les débris végétaux des laisses de mer et un peu au-dessus de celles-ci; de type banquette de Posidonies et tiges de Canne de Provence Arundo donax L., 1753 pour le premier, ou encore sous les morceaux de bois. Globalement, la pression humaine qui sévit sur les littoraux de la Méditerranée et du sud de l'Atlantique est très néfaste aux espèces spécialistes de l'estran (urbanisation, tourisme et aménagement intrinsèque des plages, dont leur nettoyage trop intensif). La France est le plus gros suppresseur de banquettes de posidonies en comparaison avec quatre autres pays méditerranéens de l'Union européenne. En effet, d'après les enquêtes effectuées, $95 \%$ des banquettes sont enlevées sur une majorité des plages et $97 \%$ de ces banquettes supprimées le sont au moins deux à trois fois par an (Otero et al. 2018). 89 \% des enlèvements sont mécaniques dont $50 \%$ avec des engins lourds, ce qui est dévastateur pour la biodiversité (Otero et al. 2018; Chauvin 2020).

\section{Coleoptera Cerambycidae et Vesperidae ("Longicornes»)}

Les Longicornes (Fig. 8) font partie des Coléoptères les plus "populaires» auprès des naturalistes, pour des raisons à la fois esthétiques (taille, élégance, couleurs) et pratiques (faciles à trouver, aisés à déterminer), comme l'indiquait déjà Colas (1974) dans son Guide de l'entomologiste. En France métropolitaine, on recense 250 espèces, chiffre qui évolue régulièrement avec la découverte d'espèces cryptiques et surtout avec l'implantation d'espèces exotiques (Brustel et al. 2002; Berger 2012; Cocquempot et al. 2019). C'est un des rares groupes de Coléoptères à avoir bénéficié de plusieurs ouvrages comprenant des clés d'identification et une iconographie complète, à l'échelle française (Villiers 1978; Bijiaoui 1986; Berger 2012) ou européenne (Bense 1995; Sama 2003). Des cartes de répartition contemporaine (période post-1970) existent pour l'ensemble des espèces, à partir de 137000 données disponibles dans l'INPN 


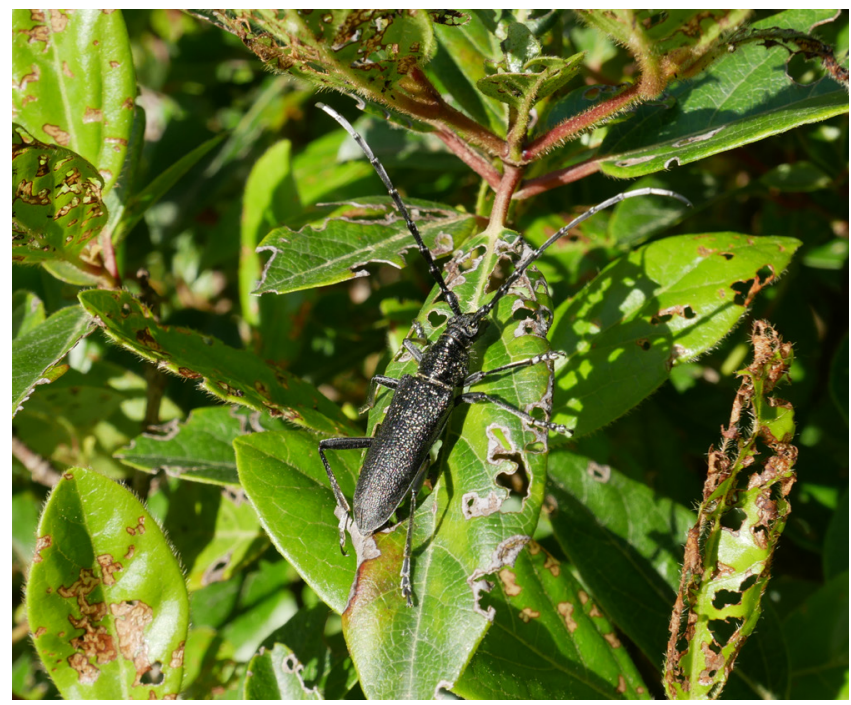

FIG. 8. - Exemple d'un coléoptère Cerambycidae: Cerambyx scopolii Fuessly, 1775. Crédit photo: O. Vellot.

(Touroult et al. 2019). La production de données est en forte hausse depuis les années 1990 et repose sur des dynamiques d'atlas locaux (e.g., Gouverneur \& Guérard 2011), des inventaires pour des gestionnaires d'espaces, des travaux de recherches sur les Insectes saproxyliques, des programmes participatifs (ex. SPIPOLL, INPN Espèces, etc.) et bien entendu sur les entomologistes amateurs. Bien que certaines espèces soient très discrètes, l'écologie et les méthodes de collecte sont connues pour quasiment tous les taxons. Le référentiel taxinomique est stable, malgré des changements réguliers dans la classification supra-spécifique (ex. pour les genres de Leptures) et pratiquement la moitié des espèces peut être qualifiée de facile à déterminer (Touroult et al. 2019).

Les Longicornes sont tous phytophages, la majorité consommant à l'état larvaire le bois des troncs, branches d'arbres et arbustes morts ou dépérissants (xylophages ou saproxylophages), les adultes étant souvent floricoles. Les Longicornes français sont souvent à large répartition, avec beaucoup d'espèces ayant une bonne capacité de dispersion ce qui leur permet de détecter et coloniser les arbres mourants. La diversité décroit du sud-est vers le nord-ouest (Vitali \& Schmitt 2016; Touroult et al. 2019). On ne compte que cinq espèces (sub)endémiques, et une seule endémique à répartition très restreinte, Brachyta borni (Ganglbauer, 1903) dans les Alpes du Sud; soit à peine plus de $2 \%$ de la richesse spécifique française du groupe (Tableau 3).

Les enjeux de conservation identifiés pour les Longicornes ne concernent pas que les espèces endémiques. Les espèces liées aux vieux arbres, notamment celles inféodées aux cavités d'arbres feuillus et celles des forêts anciennes et matures, présentent de vastes aires de répartition mais avec des distributions relictuelles et localisées. Des enjeux associés, de continuité spatio-temporelle des habitats, concernent les espèces saproxyliques à distribution boréo-alpine et celles liées à des milieux boisés menacés, comme les ripisylves et les zones humides en général. Les espèces phytophages sur

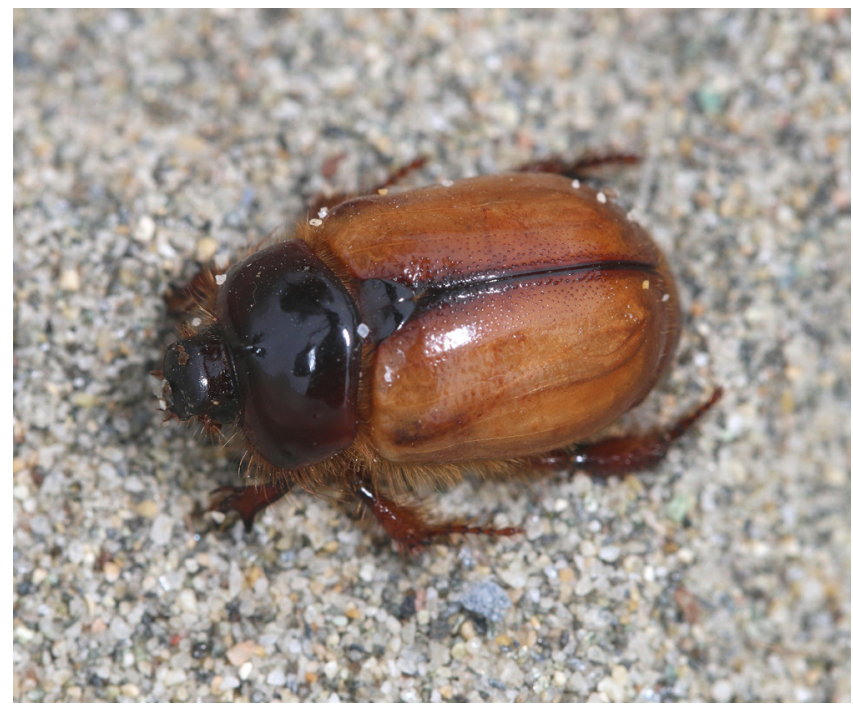

FIG. 9. - Exemple d'un coléoptère Scarabaeoidea: Calicnemis latreillii Laporte de Castelnau, 1832. Crédit photo: H. Bouyon.

plantes herbacées présentent des enjeux de conservation liées aux pelouses, prairies et garrigues. Le trait de vie lié à l'aptérisme (ou brachyptérisme) qui caractérise des espèces peu mobiles, souvent localisées comme les dorcadions, est un facteur important pour définir les espèces a priori les plus vulnérables aux changements de gestion des milieux.

Deux espèces sont protégées en France dans le cadre de la directive européenne Habitats-Faune-Flore: Rosalia alpina et Cerambyx cerdo. Ces deux espèces sont pertinentes pour la conservation de leurs milieux (Brustel et al. 2019) mais ne représentent pas, loin s'en faut, les enjeux majeurs de conservation des Longicornes de France. Dans la plupart des régions de France, le programme d'inventaire des zones clés de biodiversité (ZNIEFF) liste des Longicornes déterminants pour l'identification des ZNIEFF. Il n'existe pas de Liste rouge pour la France métropolitaine mais la nouvelle Liste rouge européenne des Coléoptères saproxyliques (Cálix et al. 2018) classe dans les catégories menacée et quasi menacées plusieurs espèces qui présentent des enjeux de conservation importants en France. De plus, le travail de Brustel (2002) identifie plusieurs Longicornes au sein de 300 espèces saproxyliques à enjeux de conservation (exigences écologiques et rareté).

\section{Coleoptera Scarabaeoidea}

Cette superfamille (au sens de Bouchard et al. 2011) comprend de nombreux groupes populaires auprès des naturalistes : les Lucanes, les Cétoines, les Bousiers, les Hannetons, etc. (Fig. 9). En France métropolitaine, on recense environ 300 espèces. L'ensemble des espèces peut être déterminé grâce à l'ouvrage de Paulian \& Baraud (1982) et à la faune d'Europe (Baraud 1992), moyennant quelques compléments pour les espèces signalées récemment. Il faut noter que la difficulté de détermination est très variable: quelques espèces sont bien caractéristiques mais il y en a aussi beaucoup délicates à déterminer (notamment parmi les Aphodiinae et Melolonthinae). Dès 1990, les espèces coprophages (les 


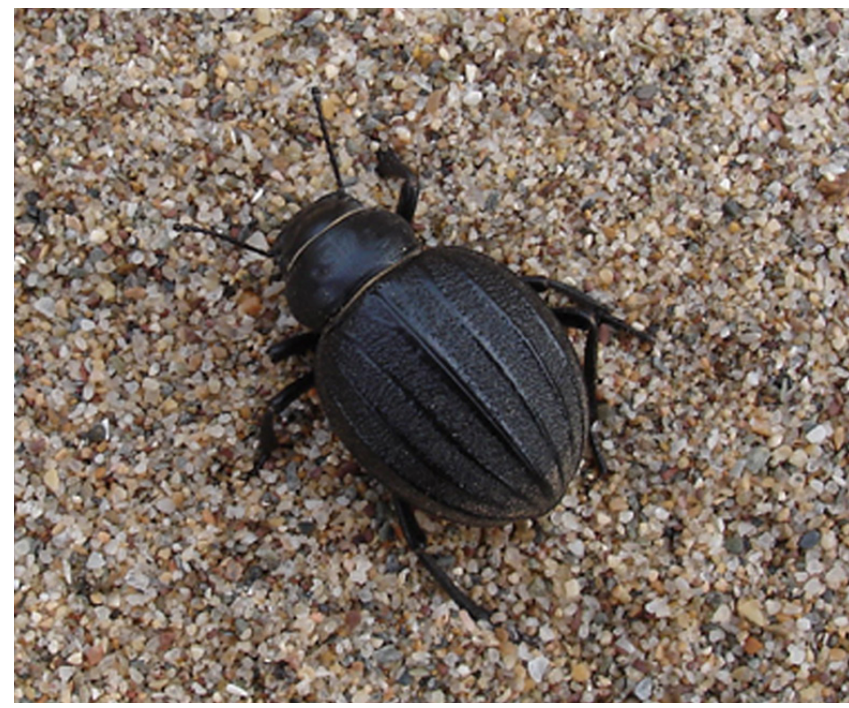

FIG. 10. - Exemple d'un coléptère Tenebrionidae: Pimelia muricata cyrnensis F. Soldati \& Rougon, 2017. Crédit photo: F. Soldati.

"Laparosticti») ont bénéficié d'un atlas national (Lumaret 1990), suivi d'un fascicule plus détaillé sur les espèces endémiques (Lumaret et al. 1996) et plus récemment d'un projet d'observatoire "ScaraB'Obs» avec un portail en ligne (https:// scarab-obs.fr/, dernière consultation le 30 décembre 2020). Les Cetoniinae ont fait l'objet, pour la plupart, d'articles dédiés à leur écologie et leur répartition (e.g., Tauzin 2007; Tassi et al. 2004). Près de 125000 données sont disponibles dans l'INPN (Tableau 3), avec de fortes variations selon les taxons: les Lucanes et Cétoines bénéficiant de nombreuses données (env. 1750 données/espèce en moyenne) et à l'inverse, les Aphodiinae s'avérant nettement moins connus (240 données/espèce).

Ce groupe comprend 17 espèces endémiques ou subendémiques, soit 5,7 \% de sa faune française; en grande partie des espèces à distribution tyrrhénienne. Cependant, l'endémisme n'est pas le seul critère d'enjeu de conservation. Trois groupes fonctionnels font l'objet de préoccupations: les espèces coprophages (et nécrophages) dans les milieux agropastoraux, les espèces psammo-halophiles du littoral et les espèces saproxyliques en lien avec les vieux arbres en forêt et hors forêt. Il s'agit parfois d'espèces à large répartition mais localisées, en raison de leurs exigences écologiques strictes. Les Scarabéides coprophages constituent un modèle largement étudié en écologie (Hanski \& Cambefort 1991). Ils assurent des fonctions clés dans les écosystèmes : enfouissement et recyclage des excréments, dispersion de certaines graines (surtout en milieu tropical) et régulation des Diptères associés aux excréments. En Europe, ces espèces sont en déclin en raison de l'utilisation à grande échelle des produits antiparasitaires vétérinaires (Lumaret 2001), des changements de pratiques pastorales, avec un déclin marqué des grosses espèces de type "rouleur" (Carpaneto et al. 2007; Numa et al. 2020). Pour les espèces saproxyliques, les Lucanidae dépendent de la quantité de bois morts et certains Cetoniinae et Trogidae se développent quasi-exclusivement dans les cavités d'arbres. Les espèces les plus exigeantes témoignent ainsi d'une continuité dans l'espace et dans le temps de la ressource en vieux arbres, devenus rares tant dans les forêts que dans les bocages. Les Melolonthinae, phytophages, paraissent présenter moins d'enjeux de conservation.

Trois espèces de ce groupe sont ciblées par la Directive Habitat-Faune-Flore (Brustel et al. 2019): deux relèvent d'une protection nationale, Osmoderma eremita et Bolbelasmus unicornis, et la troisième, Lucanus cervus, largement répandue en France, est déterminante pour le réseau Natura 2000 sans être protégée. Malgré leur importance fonctionnelle et leur déclin, aucune espèce de bousier ne figure parmi les espèces protégées (Numa et al. 2020). La récente Liste rouge méditerranéenne des bousiers (Numa et al. 2020) liste plusieurs taxons menacés ou quasi menacés qui semblent pertinents pour la France, comme Scarabeus semipunctatus (Fabricius, 1792) classé «vulnérable». De la même manière, la Liste rouge européenne des Coléoptères saproxyliques (Cálix et al. 2018) identifie des espèces à enjeux qui concernent la France.

\section{Coleoptera Tenebrionidae}

Les Tenebrionidae (Fig. 10) constituent une famille de Coléoptères diversifiée avec plus de 20000 espèces décrites dans le monde, distribuées dans environ 2300 genres (Bousquet et al. 2018). La France compte actuellement 219 espèces dont neuf introduites considérées non encore naturalisées (Soldati \& Soldati sous presse). Il s'agit ici d'un décompte raisonné, 87 espèces ayant été exclues de la faune de France car signalées de notre pays par erreur ou s'agissant d'introduction accidentelles non renouvelées.

Les connaissances sur cette famille ont considérablement augmenté depuis les années 1990. Depuis Sainte-Claire Deville (1937), 54 espèces de Tenebrionidae ont été ajoutées à la faune de France (Bouyon 2014; Soldati \& Soldati 2014, sous presse; Soldati \& Rougon 2017; Soldati et al. 2019) dont 12 espèces nouvelles pour la science décrites depuis 1958. Si l'atlas des Tenebrionidae de France métropolitaine compilait 27000 données (Soldati 2007), celles-ci ont depuis presque doublé avec l'étude de nombreuses collections supplémentaires et une pression d'échantillonnage à l'échelle nationale particulièrement forte (environ 230 données/espèce en moyenne aujourd'hui). Ajoutons que la plupart des données compilées sont très récentes, postérieures à l'an 2000.

Sur les 219 espèces de Tenebrionidae connues en France, $30,6 \%$ sont endémiques ou subendémiques dont $9,1 \%$ d'endémiques strictes (Tableaux 3, 4). Ce pourcentage relativement élevé est dû au fait que beaucoup de représentants de cette famille sont aptères et ne se déplacent quasiment pas. Ils ont souvent une aire de distribution restreinte sur le territoire (Tableau 4). Certains endémiques français sont extrêmement localisés, les endémiques corses ayant parfois une aire de distribution très limitée sur l'île (Cap Corse, Castaniccia, côte méridionale, Alta Rocca, etc.), des endémiques de France continentale ayant également une aire réduite à une montagne comme Asida christianperezi F. Soldati, 2007 sur la Montagne du Cousson (04) ou Phylan rouxi Ferrer, 2013 à haute altitude sur le Puigmal (66) (Soldati \& Soldati 2014) 
mais c'est également le cas de certains subendémiques corsosardes ou des Alpes maritimes françaises et italiennes, connus de seulement cinq à dix localités.

Les Tenebrionidae occupent des niches écologiques très variées, mais les espèces soumises à des menaces sont essentiellement celles qui appartiennent à des cortèges psammohalophiles très spécialisés des dunes littorales et des hauts de plages (Soldati 1995; Jaulin \& Soldati 2005), certaines espèces troglophiles plus ou moins synanthropes, ou les espèces sylvatiques inféodées à des dendromicrohabitats rares et très particuliers comme certains polypores ou certaines caries de cavités de vieux arbres feuillus.

Les espèces subissant la plus forte pression anthropique sont celles inféodées au littoral méditerranéen et/ou atlantique. Les impacts sont similaires à ceux qui s'exercent sur d'autres groupes comportant des spécialistes littoraux: destruction et/ou morcèlement d'habitats étroits par l'urbanisation ou d'autres aménagements (e.g., portuaires, routiers), surpiétinement lié à une fréquentation trop élevée, nettoyage trop intensif des plages (enlèvement des laisses de mer). Nombre de ces espèces sont jugées menacées, comme par exemple les méditerranéennes Heliopates littoralis Español, 1958, Phaleria acuminata Küster, 1852 et Pimelia muricata cyrnensis F. Soldati \& Rougon, 2017.

\section{Hemiptera Heteroptera Pentatomoidea}

Les Pentatomoidea (Fig. 11) forment une superfamille qui rassemble les Acanthosomatidae, Cydnidae, Pentatomidae, Plataspidae, Scutelleridae et Thyreocoridae. L'ensemble compte 8042 espèces dans le monde (Rider et al. 2018), 261 en Europe (Dusoulier comm. pers.) et 154 en France métropolitaine (Lupoli \& Dusoulier 2015). Leur étude était assez active en France à la fin du XIxe siècle (Mulsant \& Rey 1865, 1866; Puton 1881), mais le renouveau d'intérêt à leur égard est récent. Cette nouvelle dynamique autour des Pentatomoidea vient de plusieurs facteurs concomitants, se renforçant les uns les autres et émergeant seulement depuis le début des années 2000:

- la publication de faunes, listes et clés de détermination accessibles et illustrées (Derjanschi \& Péricart 2005; Dusoulier \& Lupoli 2006; Péricart 2010; Ribes \& Pagola-Carte 2013; Lupoli \& Dusoulier 2015);

- le développement de forums d'échanges ou d'applications web utilisés par les macrophotographes (le monde des Insectes, iNaturalist, INPN Espèces, etc.);

- une dynamique associative et naturaliste, notamment impulsée par Zicrona - l'association française des hétéroptéristes - qui organise une rencontre annuelle dédiée depuis 2004 (Dusoulier et al. 2020).

Le nombre de données disponibles est évalué à 135000 à la fin de l'année 2020 (Dusoulier comm. pers.). Environ $35 \%$ de ces données sont partagées sur l'INPN mais il reste encore un effort important à fournir pour saisir, rassembler et partager l'information disponible.

Les Pentatomoidea de la faune de France comptent une majorité d'espèces relativement répandues en Europe. Aucune espèce n'y est strictement endémique. On peut seulement

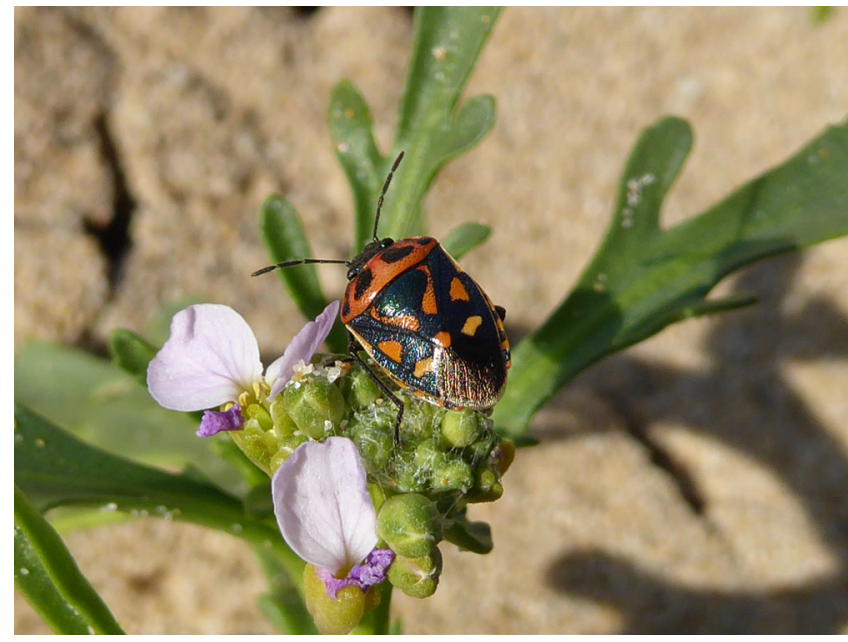

FIG. 11. - Exemple d'un hémiptère: Eurydema herbacea (Herrich-Schäffer, 1833). Crédit photo: O. Vellot.

signaler le cas de quatre espèces subendémiques: Ochetostethus melonii Rizzotti Vlach, 2000, endémique tyrrhénien, seulement connu d'un îlot de Corse et de Sardaigne (Magnien \& Ponel 2011); Holcostethus evae Ribes, 1988, limité à l'est des Pyrénées franco-espagnoles; Eurydema cyanea (Fieber, 1864), répandue dans toutes les Pyrénées franco-espagnoles; E. sea Baena, Péricart \& De la Rosa, 2004, localisée à quelques massifs calcaires du nord-est de l'Espagne et détectée récemment dans une station des Pyrénées-Orientales françaises (Sannier 2019). L'ensemble ne représente que 2,6\% des espèces (Tableaux 3, 4).

Avec leurs pièces buccales de type piqueur-suceur, les Pentatomoidea ont une alimentation exclusivement liquide. La grande majorité d'entre elles est phytophage, se nourrissant de la sève des végétaux. Selon les espèces, elles s'alimentent sur un seul genre végétal (monophagie), sur plusieurs genres d'une même famille ou sur des taxons proches (oligophagie), voire sur plusieurs ordres de plantes différents (polyphagie). Les Pentatomidae Asopinae font exception à cette règle puisque toutes les espèces sont prédatrices, s'alimentant en capturant des larves de Lépidoptères, de Coléoptères ou d'autres Hétéroptères. Le lien aux familles végétales ou aux ressources en Insectes guide en partie la distribution des espèces, mais les conditions écologiques des habitats sont également essentielles. Une large majorité d'espèces de Pentatomoidea se nourrit dans la strate herbacée ou buissonnante (Cydnidae, Pentatomidae, Plataspidae, Scutelleridae), alors que d'autres vivent sur les arbres et les arbustes (Acanthosomatidae, Pentatomidae, Scutelleridae Elvisurinae), ou encore au sol, notamment dans les sols meubles (Cydnidae, Thyreocoridae, Scutelleridae). Les prospections permettent souvent de détecter les taxons des deux premières catégories mais les espèces géophiles ont parfois des mœurs souterraines qui les rendent délicates à découvrir.

Les menaces sur les Pentatomoidea sont assez peu documentées en France. Il n'existe encore aucune liste rouge nationale ou régionale pour ce groupe. Beaucoup d'espèces ont des 


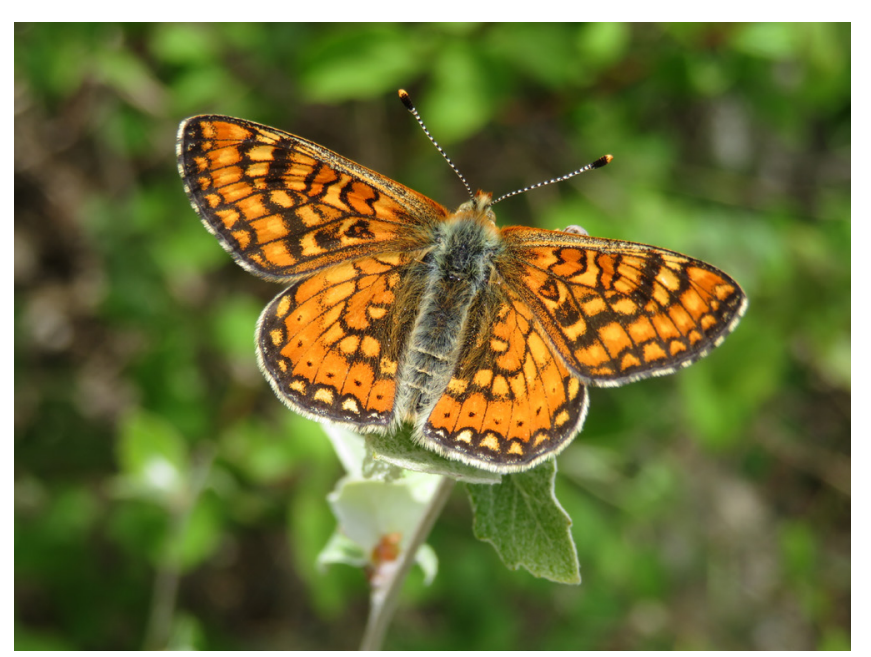

FIG. 12. - Exemple d'un lépidoptère: Euphydryas aurinia (Rottemburg, 1775) Crédit photo: É. Iorio.

exigences alimentaires, écologiques et climatiques strictes, ce qui les expose aux menaces classiques de fragmentation et de destruction de leurs habitats favorables. Par exemple, l'extrême localisation des stations de Phimodera flori Fieber, 1863, Phimodera humeralis (Dalmann, 1823) ou Eurydema sea les compromet considérablement d'un point de vue conservatoire. Plusieurs autres espèces, notamment des éléments eurosibériens, semblent régresser jusqu’à disparaître de certaines régions des plaines françaises, ce qui pourrait être la conséquence des changements globaux et de la réduction des habitats favorables. En complément du cas précédent, plusieurs espèces d'affinité méditerranéenne n'ont pas été retrouvées depuis leur signalement en France au XIx ${ }^{e}$ siècle; on peut citer les cas de Scotinophara sicula (A. Costa, 1842) en Corse, Cephalocteus scarabaeoides (Fabricius, 1803) dans les environs de Marseille, Aelia germari Küster, 1852 et Stagonomus grenieri (Signoret, 1865) dans le Midi méditerranéen. Le cas analogue le mieux documenté concerne la disparition quasi totale des populations de Ventocoris falcatus (Cyrillus, 1787), autrefois répandu dans six départements méditerranéens (Lupoli \& Dusoulier 2015). Il est encore délicat de bien comprendre les phénomènes, certainement multifactoriels, en cause dans toutes ces disparitions. Pour finir sur une note un peu plus optimiste, il semble qu'à l'inverse des cas précédents, d'autres espèces de Pentatomoidea bénéficient de bonnes capacités de dispersion; aussi est-il fréquent d'observer des cortèges diversifiés et abondants dans certaines friches ou milieux récents. Des études complémentaires sur la conservation des Pentatomoidea devraient être menées dans les années à venir.

Lepidoptera "Rhopalocères" et Zygaena

Les Rhopalocères (Fig. 12) et Zygènes représentent dans le monde environ $10 \%$ des espèces de l'ordre des Lépidoptères; 300 espèces vivent en France et un peu plus de 500 ont été notées en Europe (Leraut 2016). Aux côtés des Odonates, les papillons diurnes sont les Arthropodes les mieux connus en France, avec plusieurs millions de données (Tableau 3;
Annexe 5). L'étude des Lépidoptères y est particulièrement dynamique, avec la parution depuis 1959 de la revue Alexanor (qui a succédé à l'Amateur de papillons [1922-1937], puis à la Revue française de lépidoptérologie [1938-1957]) et la création de l'Association des Lépidoptéristes de France (ALF; anciennement Association des Lépidoptéristes parisiens) en 1992. Un autre groupe de travail national, Oreina, a vu le jour en 2007, avec la publication d'une revue trimestrielle et l'animation d'une base de données en ligne, Artemisiae. Diverses associations naturalistes sont également actives, proposant des formations sur les Rhopalocères ou réalisant des inventaires, par exemple l'Office pour les Insectes et leur Environnement (OPIE), le Groupe d'Étude des Invertébrés armoricains (GRETIA), l'Atlas entomologique régional (Nantes) (AER) ou encore les délégations régionales de la Ligue pour la Protection des Oiseaux (LPO). Les Lépidoptères diurnes bénéficient de nombreux ouvrages d'identification et d'un plan national d'actions, initialement lancé sur les Phengaris (Maculinea) (Dupont 2010a), puis élargi en 2018 à d'autres Rhopalocères (Houard \& Jaulin 2018).

Sur les 300 espèces de papillons diurnes français, 18 sont subendémiques, soit $6 \%$ du total. Au niveau spécifique, peu de subendémiques ont une répartition étroite et il n'y a aucun endémique strict (Tableau 4). Quelques sous-espèces endémiques ou subendémiques apparaissent cependant plus localisées, comme Lysandra coridon nufrellensis Schurian, 1977, inféodée à un massif montagneux en Corse.

Les Rhopalocères et Zygènes occupent de nombreux biotopes, en particulier des milieux ouverts et semi-ouverts de nature diverse (Lafranchis et al. 2015). Les habitats regroupant les espèces les plus exigeantes sur le plan écologique sont essentiellement les prairies, pelouses sèches et garrigues (e.g., Chazara briseis (Linnaeus, 1764), Coenonympha dorus (Esper, 1782) et Hyponephele lupina (O. Costa, 1836)), les prairies humides (e.g., Euphydryas aurinia aurinia (Rottemburg, 1775) et Phengaris teleius (Bergsträsser, 1779)), les tourbières (e.g., Boloria aquilonaris (Stichel, 1908) et Coenonympha tullia (O. F. Müller, 1764)) et les prairies et landes subalpines à alpines (e.g., de nombreux moirés [Erebia spp.] (Lafranchis et al. 2015)). Les milieux forestiers leur sont nettement moins propices, même si en contexte éclairci (i.e. boisements peu ou modérément denses, lisières, clairières), ils peuvent abriter quelques raretés sténotopes comme notamment Lopinga achine (Scopoli, 1763) (Sordello et al. 2013; Lafranchis et al. 2015). Certaines espèces trouvent leurs plantes-hôtes larvaires dans les ripisylves (e.g., Apatura ilia (Denis \& Schiffermüller, 1775) sur les peupliers et Nymphalis antiopa (Linnaeus, 1758) sur les saules). Peu de papillons diurnes se reproduisent dans les habitats côtiers tels que les dunes, hormis quelques ubiquistes (Lafranchis et al. 2015); mais quelques-uns peuvent y trouver localement refuge, comme Plebejus argus (Linnaeus, 1758) ou Hipparchia semele (Linnaeus, 1758). La faculté de dispersion des imagos est très variable selon les espèces. Par exemple, les déplacements courants des Phengaris tels que $P$. arion (Linnaeus, 1758), $P$. teleius et $P$. nausithous (Bergsträsser, 1779) ne dépassent pas 100 à 400 mètres. Mais la dispersion entre différents sites peut atteindre quelques kilomètres, voire une dizaine chez P. arion (Sordello et al. 2013; Nowicki et al. 2014; Baillet 
et al. 2015). D’autres taxons plus mobiles, tel Pieris brassicae (Linnaeus, 1758), peuvent parcourir plus de 35 kilomètres (Bergerot 2010), ou plus encore pour quelques migrateurs loco-régionaux ou au long cours.

Les facteurs menaçant les papillons diurnes sont bien connus (e.g., Lafranchis et al. 2015; Dewulf \& Houard 2016) : fragmentation et diminution des habitats favorables en raison de l'urbanisation et de la densification agricole, drainage, abandon du pâturage extensif et fermeture des milieux, réchauffement climatique, utilisation massive de phytosanitaires, destruction directe, etc. Des comparaisons entre données contemporaines et historiques dans certaines régions permettent d'estimer la régression ou la progression des espèces (e.g., Perrein 2012; Guilloton 2018). De nombreuses Listes rouges régionales UICN existent en plus des listes nationales et européennes: 15 des 22 régions (selon le découpage antérieur à 2016) possèdent une telle liste (https://inpn.mnhn.fr/espece/programme/ listes-rouges/RG/, dernière consultation le 15 janvier 2021).

\section{Odonata}

Les Odonates (Fig. 13), riches d'environ 6000 espèces dans le monde, comptent 143 espèces en Europe et 98 espèces en France (Boudot \& Kalkman 2015; Polette et al. 2017; Soustelle et al. 2019). Plus d'un million de données ont été obtenues sur les Odonates, soit un ratio moyen excédant 10000 données/espèce à ce jour (Tableau 3). En France, les Odonates sont l'objet d'une activité très régulière depuis les années 80 , avec la parution de la revue odonatologique Martinia depuis 1985 et la création de la Société française d'Odonatologie (SFO) en 1991 (devenue Opie-Odonates en 2019). Diverses associations naturalistes fournissent des formations sur les Libellules, comme l'Office pour les Insectes et leur Environnement (Opie), le Groupe d'Étude des Invertébrés armoricains (GRETIA) ou encore certaines délégations régionales de la Ligue pour la Protection des Oiseaux (LPO). Divers ouvrages facilitent largement la détermination des imagos et des exuvies (e.g., Dijsktra \& Lewington 2007; Doucet 2016; Grand et al. 2019). Les Odonates ont fait l'objet d'un premier plan national d'actions (Dupont 2010b), décliné dans la plupart des régions. Une deuxième version de ce plan a été présentée en consultation publique fin 2020 et a été officiellement lancée en 2021 (Houard 2020).

Sur les 98 espèces d'Odonates connues en France, aucune n'est strictement endémique ou subendémique. En élargissant un iota la notion de subendémisme pour ce seul ordre, quatre espèces sont subendémiques franco-ibériques: Platycnemis acutipennis Selys, 1841, P. latipes Rambur, 1842, Gomphus graslinii Rambur, 1842 et Macromia splendens (Pictet, 1843); une espèce est endémique des îles italiennes (e.g., archipel toscan, Sardaigne et Sicile), de Corse et de Malte: Ischnura genei (Rambur, 1842) (Boudot \& Kalkman 2015). L'ensemble représente $5,1 \%$ de la faune française.

Il faut distinguer les Anisoptères et les Zygoptères sur le plan des capacités de dispersion. La plupart des premiers, au vol puissant, peut voler sur des dizaines ou centaines de kilomètres voire davantage (Borisov 2012; Lambret \& Boudot 2013). Les seconds ont généralement une dispersion beaucoup plus faible, de quelques centaines de mètres chez certains Coenagrion (Rou-

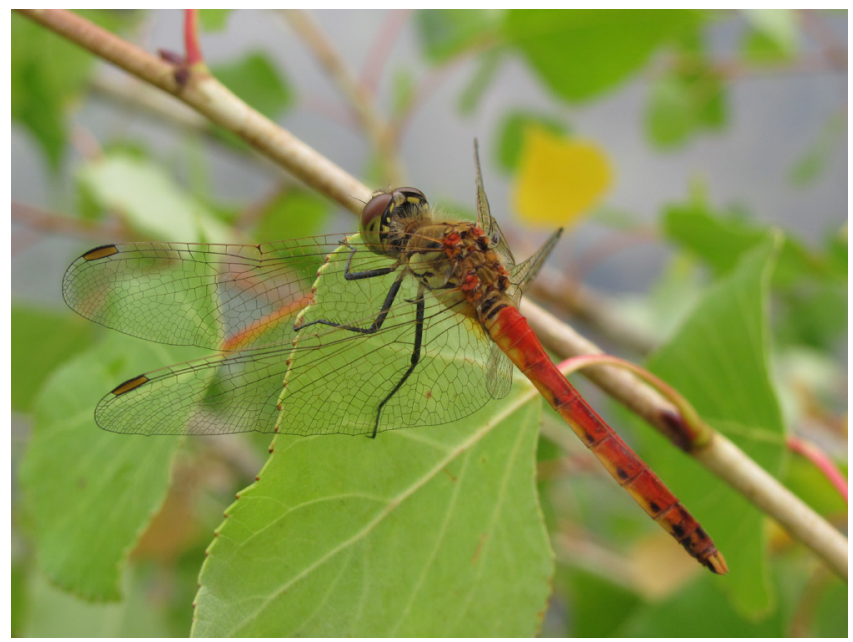

FIG. 13. - Exemple d'un odonate: Sympetrum depressiusculum (Selys, 1841). Crédit photo: É. Iorio.

quette $\&$ Thompson 2007) à quelques kilomètres ou un peu plus chez les Lestes, les Sympecma ou les Calopteryx (Stettmer 1996; Manger \& Dingemanse 2007; Monnerat \& Maibach 2014). Les Libellules, insectes hémimétaboles, effectuent l'essentiel de leur cycle vital dans l'eau (soit tout leur cycle larvaire) et de ce fait, sont fortement dépendantes des milieux aquatiques. Une espèce, Lestes macrostigma (Eversmann, 1836), est considérée comme privilégiant les mares saumâtres temporaires; les autres étant propres aux eaux douces même si quelques-unes tolèrent une légère salinité (Dumont \& Dumont 1969; Hinnekint 1987). Certaines Libellules sont relativement sténoèces et requièrent la combinaison de deux ou plusieurs facteurs adéquats, tels que la présence ou (quasi)absence de courant, un assec ou non, la granulométrie des sédiments, la qualité et la limpidité de l'eau, les cortèges de végétation associée pour les Zygoptères à pontes endophytiques, ou encore la présence d'une ripisylve (pour le contexte ombragé et/ou pour l'existence de lacis racinaires immergés) (e.g., Schridde \& Suhling 1994; Leipelt \& Suhling 2001; Purse 2002; Matushkina \& Gorb 2002). D'autres espèces sont au contraire euryèces et peuvent faire preuve d'une grande plasticité écologique, pouvant même se reproduire dans des eaux polluées (Villalobos-Jiménez et al. 2016). Les capacités bio-indicatrices des Odonates ont été bien étudiées (Corbet 1993; D’Amico et al. 2004).

Les menaces pesant sur les Libellules sont largement connues (e.g., Poitou-Charentes Nature 2009) : destruction et fragmentation des habitats, artificialisation des cours d'eau, curage ou comblement des mares, pollution, piétinement et eutrophisation par les troupeaux, dénaturation ou entretien inadapté des berges de rivières, de mares ou d'étangs, espèces invasives antagonistes aux Libellules et à leurs habitats (e.g., Procambarus clarkii (Girard, 1852)), fermeture des milieux pour les espèces héliophiles (e.g., Coenagrion mercuriale), réchauffement climatique, etc. Le degré de menace des espèces de France métropolitaine est bien connu grace à la publication de nombreuses Listes rouges régionales labellisées UICN en plus des listes nationales et européennes: pas moins de 15 des 22 régions (en 


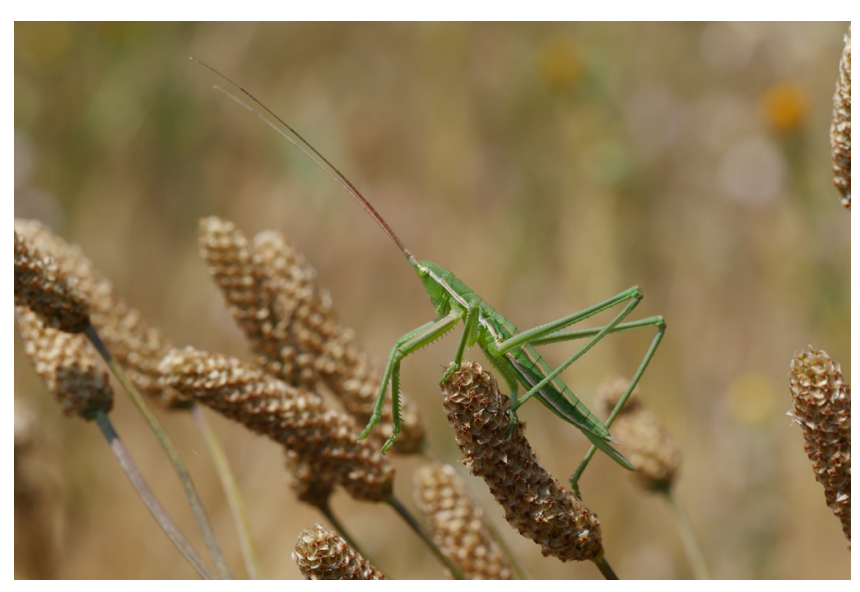

FIG. 14. - Exemple d'un orthoptère: Saga pedo (Pallas, 1771). Crédit photo: O. Vellot.

prenant le découpage antérieur à 2016) possèdent une telle liste (https://inpn.mnhn.fr/espece/programme/listes-rouges/RG/, dernière consultation le 15 janvier 2021). Leurs enjeux de conservation sont donc aisés à déterminer en croisant ces listes avec d'autres critères tels que répartition, écologie et facultés de dispersion.

\section{Orthoptera}

Lordre des Orthoptères (Fig. 14) compte près de 29000 espèces valides dans le monde (Cigliano et al. 2021), dont 1082 en Europe (Hochkirch et al. 2016) et 232 en France métropolitaine (Gargominy et al. 2020b). Un peu plus d'un million de données d'Orthoptères est disponible pour la France (Tableau 3; Annexe 6), ce qui place cet ordre autrefois délaissé parmi les Insectes les plus étudiés aujourd'hui. L'accélération très importante de la connaissance depuis le début des années 2000 est due à trois facteurs principaux :

- la publication d'ouvrages récents, le plus souvent illustrés, sur la détermination et l'écologie des espèces (Bellmann \& Luquet 1995; Defaut 1999a, b; Coray \& Thorens 2001; Baur et al. 2006; Defaut \& Morichon 2015; Sardet et al. 2015);

- la dynamique naturaliste des associations, et notamment celle de l'ASCETE qui regroupe tous les spécialistes des Orthoptères depuis plus de vingt ans;

- la publication d'une liste rouge - non labellisée IUCN au niveau national (Sardet \& Defaut 2004) et des listes rouges labellisées IUCN au niveau européen (Hochkirch et al. 2016) et dans neuf régions des 22 "anciennes » régions (découpage administratif antérieur à 2016) (https://inpn.mnhn.fr/espece/ programme/listes-rouges/RG/, dernière consultation le 15 janvier 2021). Malgré ces développements importants dans la connaissance, quelques groupes (Tetrigidae, Rhaphidophoridae, Myrmecophilidae, Sphingonotus, etc.) demeurent encore largement sous-prospectés en raison d'une identification et/ ou d'une détection complexe.

La faune des Orthoptères de France comprend 22 espèces endémiques ( $9,5 \%)$ et 25 subendémiques (10,7\%) (Tableau 4), la plupart d'entre elles étant corses ou provençales pour les premières, alpiennes ou pyrénéennes pour les secondes. Parmi les endémiques, certaines sont très localisées comme Prionotropis rhodanica Uvarov, 1923, limité à moins de $16 \mathrm{~km}^{2}$ d'occupation (AOO) en Crau sèche (Hochkirch et al. 2016) ou Anonconotus mercantouri Galvagni \& Fontana, 2003 qui ne vit que dans quelques stations de la haute vallée de la Vésubie. D'autres endémiques, notamment en Corse, ont une distribution limitée à quelques secteurs collinéens ou montagnards comme Antaxius bouvieri Chopard, 1924 dans les massifs du Cintu et du Ritondu. Par ailleurs, le renouveau de l'étude des Orthoptères a permis la description de plusieurs taxons au cours des vingt dernières années, même s'il demeure des incertitudes sur le statut exact de certains d'entre eux.

Le régime alimentaire des Orthoptères est variable selon les groupes. Les Sauterelles et Grillons sont majoritairement omnivores, s'alimentant de petits Insectes mous et de végétaux; seules les plus grandes espèces (Saga, Decticus, Tettigonia, etc.) sont carnivores en se nourrissant d'Insectes plus gros. Les Criquets sont phytophages et s'alimentent principalement de graminées. Dans leur ensemble, ils colonisent tous les habitats ouverts à semi-ouverts (pelouses, prairies, rocailles, landes, marais, etc.), les écotones (lisières, haies, etc.) ou encore les forêts et boisements clairs. Ils sont présents dans différentes strates, de la litière (Grillons, Tétrix) à la canopée (Phanéroptères), avec une abondance et une diversité plus forte dans les strates herbacées et arbustives. Cette diversité fonctionnelle fait que les Orthoptères sont souvent utilisés comme indicateurs écologiques, en raison de leur sensibilité à la structure de la végétation, l'hygrométrie des sols et à la «qualité de préservation" des habitats ouverts (Bazelet 2011). De plus, ils présentent l'avantage d'être assez facilement détectables, à vue ou grâce à l'écoute de leurs stridulations, de jour et/ou de nuit.

Les facteurs de menaces des Orthoptères demeurent encore imparfaitement connus (Hochkirch et al. 2016), même s'il est clair que la destruction des milieux nécessaires aux espèces sténotopes apparaît principale, notamment pour celles des pelouses substeppiques rocailleuses, des marais et tourbières, des vieilles landes à ajoncs et bruyères ou des massifs dunaires. Si cette fragmentation et réduction des surfaces d'habitats favorables par les différentes formes d'agricultures (traditionnelles, fruitières, viticulture, arboriculture, etc.), les infrastructures industrielles et commerciales (installations éoliennes, photovoltaïques, stations de ski, etc.) ou l'urbanisation sont probablement la première cause de menace, d'autres effets comme ceux des changements globaux, des incendies ou des modes de pâturage sont plus délicats à évaluer. À ce titre, un certain nombre d'espèces de haute altitude et leurs habitats n'apparaissent pas directement menacées, alors que leur remontée altitudinale est préoccupante sur le long terme. L'étude récente de Couturier et al. (2020) montre en effet une remontée altitudinale moyenne de l'ordre de 150 mètres pour 27 espèces d'Orthoptères des Alpes du Sud au cours des 30 dernières années. En secteur de plaine, une étude biohistorique (Dusoulier 2006) avait permis d'évaluer l'érosion du nombre d'espèces à l'échelle d'un territoire; selon le périmètre considéré (ensemble du Massif armoricain ou Bretagne), le constat était que 9,5 à 13,2\% des espèces avaient disparu au cours des 50 dernières années. 
35

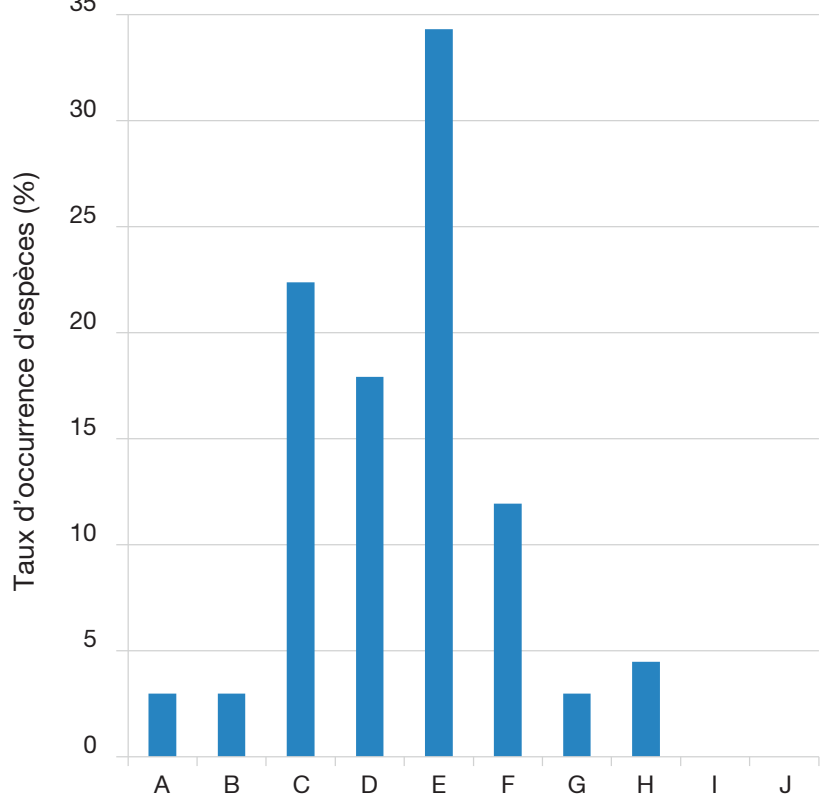

FIG. 15. - Taux d'occurrence par type d'habitat pour les 45 espèces à enjeux remarquables définies en cumulant les Lépidoptères diurnes, les Odonates et les Orthoptères. A, habitats marins; B, habitats côtiers; C, eaux de surface continentales; $\mathbf{D}$, tourbières hautes et bas-marais; $\mathbf{E}$, prairies, terrains dominés par des herbacées non graminoïdes, des mousses ou des lichens; $\mathbf{F}$, landes, fourrés et toundras; $\mathbf{G}$, boisements, forêts et autres habitats boisés; $\mathbf{H}$, habitats continentaux sans végétation ou à végétation clairsemée; I, habitats agricoles, horticoles et domestiques régulièrement ou récemment cultivés; J, zones bâties, sites industriels et autres habitats artificiels.

\section{Constat général}

En comparant les caractéristiques des trois groupes déjà communément pris en compte dans les études d'impact (Rhopalocères, Odonates et Orthoptères), avec celles des six groupes complémentaires proposés, les premiers présentent des caractéristiques distinctes des seconds (Tableaux 3, 4). Ils comprennent une quantité de données disponibles par espèce beaucoup plus élevée, ils disposent de nombreuses Listes rouges régionales, mais leur taux d'espèces endémiques est nettement plus faible que trois des six groupes complémentaires (Chilopodes, Isopodes terrestres et Coléoptères Tenebrionidae).

\section{SÉLECTION DE QUINZE ESPÈCES À ENJEUX FORTS}

OU MAJEURS DE CONSERVATION POUR LES GROUPES ÉTUDIÉS ET ÉLÉMENTS DE SYNTHÈSE

\section{5 espèces à enjeux remarquables de conservation}

La consultation des références bibliographiques synthétisées dans la deuxième partie des résultats, associée à des échanges entre experts de chaque groupe sur la base des Tableaux 1 et 2 et à la prise en compte de données inédites de répartition provenant des bases, a permis de déterminer 135 espèces à enjeux remarquables de conservation. Parmi elles, 64 constituent des enjeux de niveau majeur et 71 sont de niveau fort. Ces espèces et leurs caractéristiques sont rassemblées dans l'Annexe 2.

Par ailleurs, l'application des critères Liste rouge sur les 15 Chilopodes sélectionnés fait apparaître que trois espèces seraient "en danger d'extinction" $(\mathrm{EN})$ et six seraient «vulné-

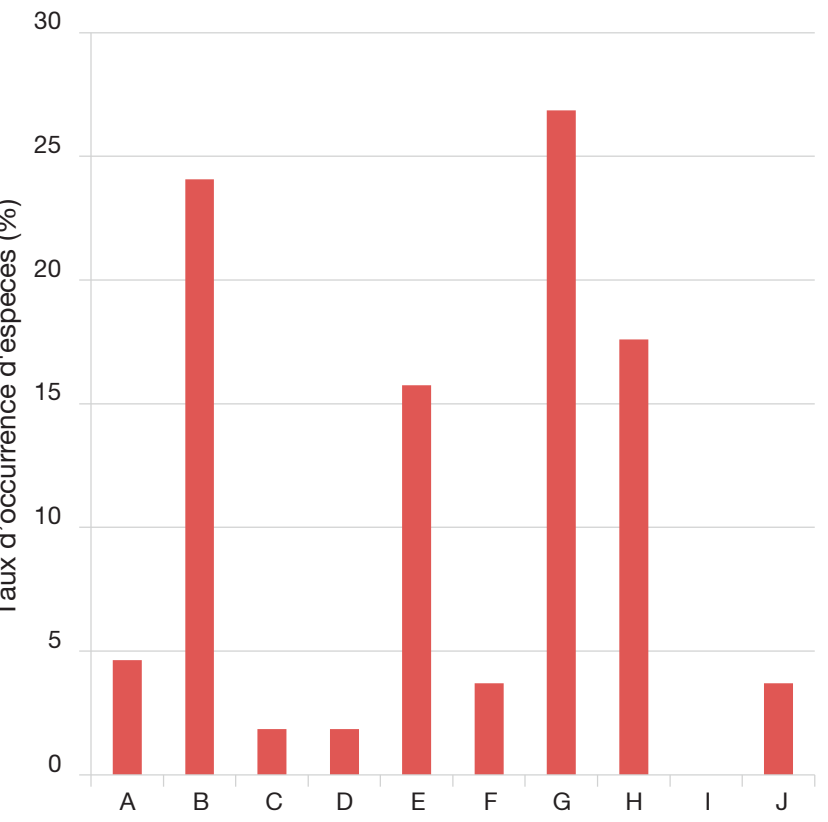

FIG. 16. - Taux d'occurrence par type d'habitat pour les 90 espèces à enjeux remarquables définies pour les six autres groupes d'Arthropodes étudiés. A, habitats marins; $\mathbf{B}$, habitats côtiers; C, eaux de surface continentales; $\mathbf{D}$, tourbières hautes et bas-marais; $\mathbf{E}$, prairies, terrains dominés par des herbacées non graminoïdes, des mousses ou des lichens; $\mathbf{F}$, landes, fourrés et toundras; $\mathbf{G}$, boisements, forêts et autres habitats boisés; $\mathbf{H}$, habitats continentaux sans végétation ou à végétation clairsemée; I, habitats agricoles, horticoles et domestiques régulièrement ou récemment cultivés; $\mathbf{J}$, zones bâties, sites industriels et autres habitats artificiels.

rables» (VU). Deux autres disposent de "données insuffisantes» (DD) et trois autres sont de "préoccupation mineure» (LC); ces cinq dernières étant des espèces (sub)endémiques $(\mathrm{n}=3)$ et/ou correspondant à d'autres critères d'enjeux (Tableau 2). L'évaluation provisoire est fournie en Annexe 3 et constitue une base en attendant une future Liste rouge complète pour ce groupe.

Synthèse par grands types d'habitats selon la typologie EUNIS Les pourcentages d'occurrences des 135 espèces à enjeux remarquables par grand type d'habitat sont fournis: d'une part pour les trois groupes d'Arthropodes les plus couramment étudiés dans les études d'impact, soit les Lépidoptères diurnes (Rhopalocères et Zygaena), les Odonates et les Orthoptères (Fig. 15); d'autre part, pour les six autres groupes retenus dans le présent article: Chilopodes, Isopodes terrestres, Coléoptères "Longicornes », Scarabaeoidea et Tenebrionidae, Hétéroptères Pentatomoidea (Fig. 16).

La Figure 15 traduit les mêmes tendances que la Figure 5, qui concernait les pourcentages d'espèces d'Arthropodes à forts enjeux de conservation par grand type d'habitat dans les 50 études d'impacts examinées. Dans la Figure 15, le type E d'EUNIS ("prairies et terrains dominés par des herbacées non graminoïdes, des mousses ou des lichens") rassemble de nouveau la part la plus importante des espèces à enjeux forts ou majeurs (34,3\%), ici celles que nous avons définies pour les Lépidoptères diurnes, les Odonates et les Orthoptères. Cette part est plus de dix fois supérieure à celle concernant le type $G$ 


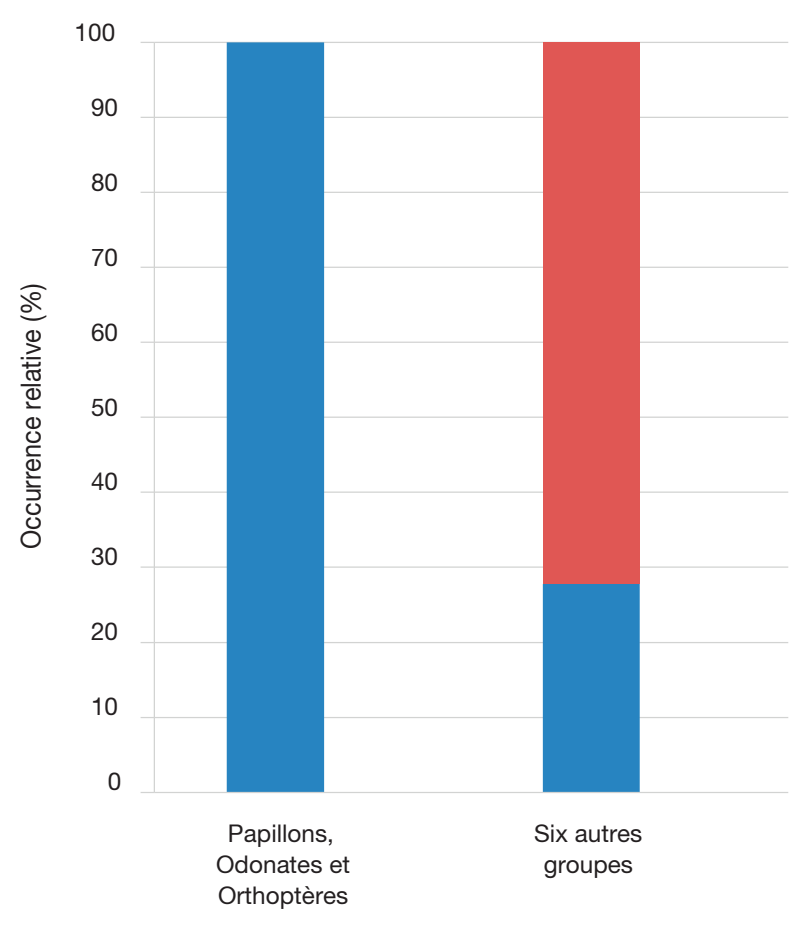

Espèces épigées de milieux à végétation clairsemée, d'éboulis, etc.

Espèces cavernicoles et endogées uniquement

FIG. 17. - Occurrence relative des espèces épigées et cavernicoles dans le type $\mathrm{H}$ d'EUNIS (habitats continentaux sans végétation ou à végétation clairsemée), d'un côté pour les papillons diurnes, Odonates et Orthoptères, de l'autre pour les six autres groupes.

(«boisements, forêts et autres habitats boisés») (3\%). Comme sur la Figure 5, le deuxième type avec le plus d'enjeux est le C ("eaux de surface continentales») (22,4\%). On note que le type $\mathrm{D}$ («tourbières hautes et bas-marais) est ici le troisième (17,9\%) devant le F («landes, fourrés et toundras») (11,9\%); les deux étant ex aequo sur la Figure 5. Bien qu'ici, les types A («milieux marins»), B («milieux côtiers», et H («Habitats continentaux sans végétation ou à végétation clairsemée») ne soient pas complètement dépourvus d'espèces à enjeux, les pourcentages d'occurrences restent extrêmement bas (3\% pour les deux premiers, 4,5\% pour le troisième).

La Figure 16 montre des tendances très différentes des Figures 5 et 15 : l'habitat rassemblant de loin le plus d'espèces à enjeux fort ou majeur est le type $\mathrm{G}$, forestier (26,9\%), suivi d'assez près par le type B (habitats côtiers) $(24,1 \%)$. La plupart des autres habitats different aussi sensiblement: le type A (habitats marins) et surtout le type $\mathrm{H}$ (habitats continentaux sans végétation) sont mieux représentés, les deux passant respectivement de 0 à 4,6 \% et de 0 à 17,6 \% par rapport à la Figure 5 ; le second présentant près de quatre fois plus d'occurrences d'espèces à enjeux que sur la Figure 15. Ces occurrences diminuent ici nettement dans les habitats prairiaux (E), les landes et fourrés (F) et plus encore les milieux dulcicoles (C).

Rappelons que le type $\mathrm{H}$ rassemble les grottes, avens et milieux souterrains superficiels (MSS), et des habitats naturels purement épigés (surface des éboulis, milieux rocailleux ou
25

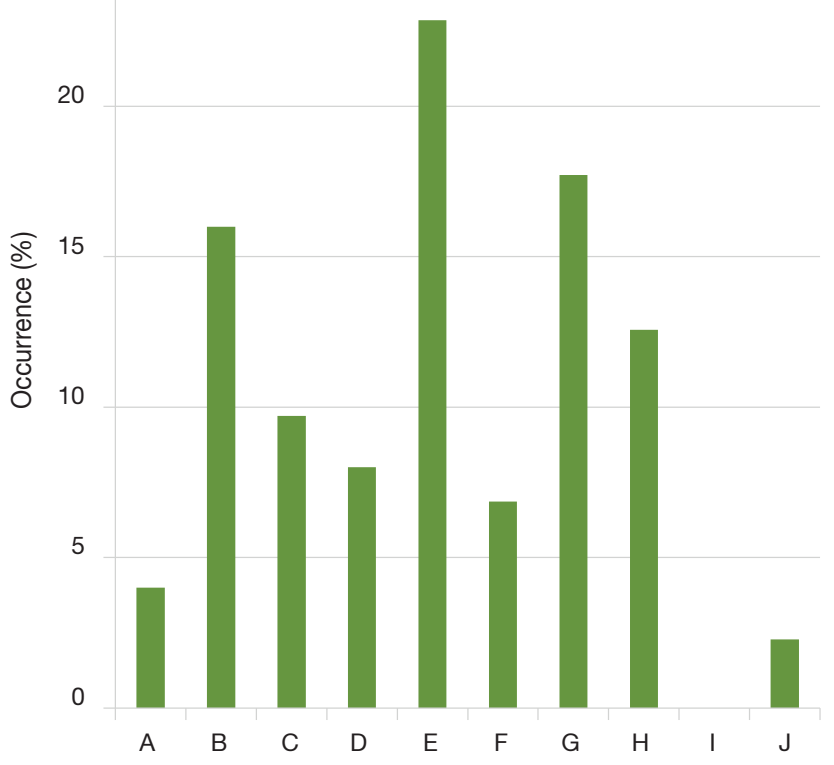

FIG. 18. - Taux d'occurrence par type d'habitat pour la totalité des 135 espèces à enjeux remarquables des neuf groupes confondus. A, habitats marins; B, habitats côtiers; C, eaux de surface continentales; $\mathbf{D}$, tourbières hautes et bas-marais; E, prairies, terrains dominés par des herbacées non graminoïdes, des mousses ou des lichens; $\mathbf{F}$, landes, fourrés et toundras; $\mathbf{G}$, boisements, forêts et autres habitats boisés; $\mathbf{H}$, habitats continentaux sans végétation ou à végétation clairsemée; $\mathbf{I}$, habitats agricoles, horticoles et domestiques régulièrement ou récemment cultivés; $\mathbf{J}$, zones bâties, sites industriels et autres habitats artificiels.

parois rocheuses à végétation très clairsemée, etc.). On constate aussi une disparité entre d'un côté les papillons diurnes, les Odonates et les Orthoptères qui n'ont guère d'espèces cavernicoles (les Orthoptères troglophiles du genre Dolichopoda ne ressortent pas en enjeux forts et contiennent encore des ambiguïtés taxinomiques), et de l'autre, une occurrence relative de $72,2 \%$ en faveur des espèces cavernicoles dans les six autres groupes ( $\mathrm{n}=13$ sur 18) (Fig. 17); les Chilopodes, les Isopodes terrestres et les Coléoptères Tenebrionidae rassemblant la totalité des troglobiontes et troglophiles.

La Figure 18 est une synthèse comparable à celle des Figures 15 et 16, mais en totalisant les 135 espèces à enjeux forts ou majeurs de tous les groupes étudiés. L'ensemble est intermédiaire entre les Figures 15 et 16 ou entre les Figures 5 et 16, avec le type E d'EUNIS qui reste dominant mais dans des proportions bien moindres et une nette progression d'autres habitats absents ou sous-représentés dans les Figures 5 et 15 (B, G, H et de façon moindre A). Les types I ("habitats agricoles, horticoles et domestiques régulièrement ou récemment cultivés») et J («zones bâties, sites industriels et autres habitats artificiels»), qui rassemblent les habitats fortement anthropisés, contiennent peu d'espèces à enjeux.

\section{Espèces réglementaires absentes des forts enjeux,} avec zoom sur le cas de Zerynthia polyxena

Plusieurs espèces réglementaires de papillons diurnes et d'Odonates ne répondent pas à suffisamment de critères biologiques de sélection d'espèces à enjeux de conservation 
pour être retenues (Tableau 2), ni ne sont reconnues comme menacées dans les Listes rouges nationales. Seulement $25 \%$ des espèces protégées de Rhopalocères et Zygaena $(\mathrm{n}=7 / 28)$ et $33,3 \%$ de celles d'Odonates $(n=4 / 12)$ figurent dans nos listes d'enjeux; mais quand même 66,6\% de celles d'Orthoptères (2/3) (Annexe 2). À l'opposé, huit Rhopalocères et Zygènes, 10 Odonates et 13 Orthoptères que nous avons définis comme étant d'enjeux forts ou majeurs de conservation sont absents des listes réglementaires nationales et/ou européennes.

Il est même des espèces qui ne répondent à aucun objectif d'enjeu de conservation. Parmi elles, la Diane est le cas le plus notable. Zerynthia polyxena n'est pas menacée, que ce soit au niveau national (UICN France \& MNHN 2012) ou au niveau régional (Bence 2014; Baillet \& Guicherd 2018; Louboutin et al. 2019). Louboutin et al. (2019) font le constat que cette espèce peut survivre localement dans divers contextes rudéraux comme les bords de cultures et les bords de route. Puissauve (2009) a aussi observé la Diane en contexte agricole. Aubin et al. (2019) mentionnent qu'une réelle progression de $Z$. polyxena a été observée au cours des quarante dernières années dans les Alpes-de-Haute-Provence, en plus de la découverte de nombreuses stations ailleurs depuis dix ans.

Nos propres observations de chenilles et d'œufs de $Z$. polyxena illustrent son caractère eurytope: même si la majorité est située en milieu prairial, de nombreux autres habitats sont aussi documentés, notamment forestiers (lisières forestières, bois clairs, abords de chemins forestiers, ripisylves, haies arborées, etc.) (Fig. 19). Bien qu'une partie non négligeable de nos données concerne des milieux "naturels" plus ou moins vastes, plus de la moitié $(52,9 \%)$ se rapporte à des biotopes fragmentés et exigus en contexte anthropisé, i.e. à proximité immédiate ou au cœur de parcelles agricoles, viticoles ou (péri)urbaines, au bord de routes (M. Tardy, É. Iorio \& F. Dusoulier comm. pers.). La principale plante-hôte est Aristolochia rotunda L. En milieu plus xérophile, la Diane peut s'accommoder pour son cycle reproducteur et larvaire d'autres aristoloches, comme notamment d'A. pistolochia Linnaeus, 1763 mentionnée par Aubin et al. (2019); un fait constaté 16 fois par nousmêmes (M. Tardy, É. Iorio \& F. Dusoulier comm. pers.).

\section{Réexamen critique de trois types d'habitats rencontrés} à l'aide des six nouveaux groupes retenus

Nous proposons ici de prendre en compte les espèces avérées ou potentielles des "nouveaux " groupes, pour trois exemples inspirés d'études d'impacts examinées dans l'échantillon de 50 et ayant été statués à l'origine comme de faible niveau d'enjeu pour les Arthropodes. Nous utilisons la bibliographie utile pour les groupes concernés (catalogues, atlas, bases de données et articles écologiques évoqués dans les présentations générales) et le dire d'expert:

- plage sableuse relativement préservée dans le Var, avec banquettes de posidonies et nombreux galets au sol, et dunes blanches, même reliques et peu étendues, à Ammophila arenaria (A et B d'EUNIS) : Chilopodes Henia bicarinata (Meinert, 1870) et Tuoba poseidonis (face inférieure des galets et des bois flottés humidifiés par l'eau salée, en contact étroit

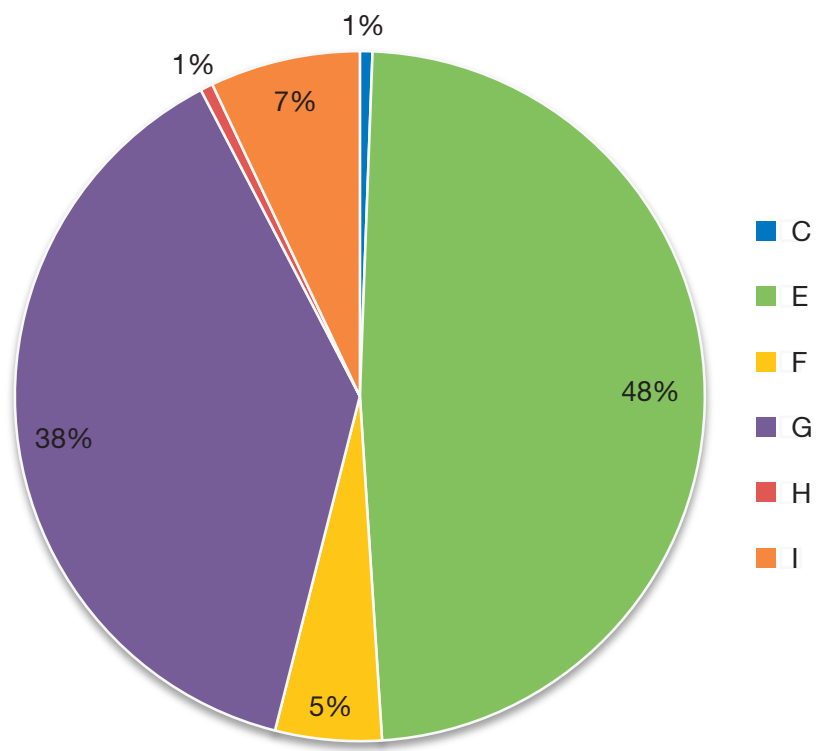

FIG. 19. - Taux d'occurrence des chenilles (sur Aristolochia spp.) et œufs (sur A. rotunda L.) de Zerynthia polyxena (Denis \& Schiffermüller, 1775) par type d'habitat du niveau 1 d'EUNIS, données de M. Tardy, E. lorio et F. Dusoulier ( $n$ occurrences $=341 ; n$ chenilles et œufs $=1137$ ). $\mathbf{C}$, eaux de surface continentales; E, prairies, terrains dominés par des herbacées non graminoïdes, des mousses ou des lichens; $\mathbf{F}$, landes, fourrés et toundras; G, boisements, forêts et autres habitats boisés; $\mathbf{H}$, habitats continentaux sans végétation ou à végétation clairsemée; I, habitats agricoles, horticoles et domestiques régulièrement ou récemment cultivés.

avec un substrat plus fin), deux enjeux halophiles majeurs; Isopodes Tylos ponticus Grebnitzky, 1874 (plages de graviers et galets), Buchnerillo littoralis Verhoeff, 1942 et Trichoniscus halophilus (dans les banquettes de posidonies), deux enjeux forts et un enjeu majeur; Coléoptères Tenebrionidae Phaleria acuminata (sous les laisses de mer) et Pimelia muricata cyrnensis (sur le sable des dunes, entre les touffes d'oyat), deux espèces psammo-halophiles à enjeux majeurs.

- hêtraie-sapinière montagnarde (> 500-780 m d'altitude) au stade futaie au sud du Massif vosgien (G d'EUNIS) : Chilopodes Lithobius pelidnus Haase, 1880 et L. dentatus C. L. Koch, 1844 (dans la litière de feuilles et sous les pierres en contact étroit avec le sol), deux enjeux forts; Coléoptère Ceruchus chrysomelinus (Hochenwarth, 1785) (résineux en décomposition, saproxylophage), enjeu majeur.

- forêt dominée par une sapinière-hêtraie (> 800-1040 m) dans l'Aude (G d'EUNIS): Chilopode Lithobius ribauti Chalande, 1907 (surtout sous la mousse, aussi sous la litière et les pierres), enjeu fort; Coléoptères Aesalus scarabaeoides (Panzer, 1795) (gros bois cariés humides) et éventuellement Liocola marmorata (Fabricius, 1792) (terreau et aubier carié des cavités de divers vieux arbres feuillus), deux enjeux forts; plus Rhysodes sulcatus (Fabricius, 1787) de l'Annexe 2 de la Directive Habitats-Faune-Flore.

Le cas de la plage varoise se démarquerait donc comme un habitat disposant d'un niveau global d'enjeu devant être positionné à majeur, particulièrement étayé par un nombre important d'espèces fortement menacées, dont plusieurs déjà avérées sur cette plage. La sapinière-hêtraie audoise serait au 
moins à niveau global fort pour les Arthropodes. Enfin, la hêtraie-sapinière vosgienne nécessiterait des prospections dédiées pour s'assurer de la présence des espèces à enjeux potentielles; mais un enjeu globalement élevé pour les Arthropodes est envisageable.

\section{DISCUSSION}

\section{QUELS HABITATS ET ENJEUX DE CONSERVATION} REFLĖTENT LES GROUPES ACTUELLEMENTS ÉVALUÉS DANS LES ÉTUDES D'IMPACT?

Les Lépidoptères diurnes (Rhopalocères et Zygaena), les Odonates et les Orthoptères sont de loin les Arthropodes les mieux connus en France actuellement. L'activité entomologique est intense depuis longtemps sur les premiers et cela n'est donc guère surprenant. En revanche, l'activité sur les Odonates s'est beaucoup développée à partir des années 1980 et celle sur les Orthoptères à partir des années 1990. Ces derniers montrent une évolution exceptionnelle de leurs données de distribution postérieures à 2000: un peu plus de 40000 données en 2003 (Voisin 2003), vingt-cinq fois plus aujourd'hui. La prise en compte progressive de ce groupe par les bureaux d'études dans les études d'impact dès le milieu des années 2000, suivant la "Liste rouge» de Sardet \& Defaut (2004), n’y est certainement pas étrangère, en parallèle des dynamiques naturalistes et associatives.

Jusqu’à présent, le volet «Insectes et autres Arthropodes » des diagnostics écologiques menés dans le cadre des études d'impact environnementales s'est systématiquement focalisé sur ces trois groupes; avec très secondairement, l'ajout ponctuel des Névroptères Ascalaphidae dans certains secteurs géographiques (Midi de la France). Les seuls autres cas régulièrement étudiés ont été limités aux espèces de Coléoptères à enjeux réglementaires. Toujours sur la base de ces enjeux réglementaires, quelques Lépidoptères hétérocères ont aussi été recherchés selon le secteur géographique concerné. On constate donc que sur le plan taxinomique, le schéma de ces études réglementaires suit celui des protections évoquées en introduction : la représentation est incomplète dans les études d'impact, puisque seuls les papillons diurnes et deux ordres sont étudiés en totalité au sein des Arthropodes continentaux, tandis que la plupart des papillons nocturnes et 23 ordres sont omis (ou limités à quelques espèces protégées) chez les Insectes et que l'ordre des Isopodes, les quatre classes de Myriapodes et la classe des Arachnides sont totalement oubliés. Le cas des Orthoptères est remarquable puisqu'ils sont bien pris en compte dans les études d'impacts alors qu'ils abritent peu d'espèces protégées. Ceci témoigne de l'effet de la disponibilité de connaissances (atlas, clés), de listes d'espèces à enjeux et des dynamiques d'acteurs.

Cependant, les moyens et le temps alloués aux études d'impact étant forcément limités, on peut d'emblée arguer à raison qu'il serait impossible d'aborder tous les Arthropodes continentaux, en raison de l'extrême diversité et complexité de cet embranchement. Pour un site de superficie moyenne (quelques dizaines d'hectares), cela demanderait l'intervention de plusieurs dizaines de spécialistes complémentaires et des centaines d'heures de travail.
Cette réalité conduit à devoir faire des choix et à se restreindre à certains groupes, comme dans certains des 50 diagnostics écologiques examinés :

- «En raison de cette diversité spécifique trop importante, il est impossible de les considérer dans leur intégralité. De fait, il convient de faire un choix quant aux groupes étudiés. Ainsi, les inventaires concernent prioritairement les groupes contenant des espèces inscrites sur les listes de protection nationales, aux annexes de la Directive Habitats, ainsi que les taxons endémiques, en limite d'aire ou menacés (Listes rouges).» (Naturalia 2017)

- «Les groupes d'Insectes recherchés sont les Odonates (Libellules), les Orthoptères (Criquets, Sauterelles et Grillons), les Mantes et Phasmes, les Rhopalocères (papillons de jour), les Névroptères (Fourmilions et Ascalaphes), ainsi que quelques gros Coléoptères saproxylophages. Ce sont des groupes qui ont pour avantage d'être représentatifs de l'ensemble des groupes d'Insectes et d'être bien connus." (Biotope 2018)

- "L'inventaire entomologique a été axé sur trois ordres d'Insectes : les Rhopalocères (papillons de jour), les Odonates (Libellules) et les Orthoptères (Criquets, Sauterelles et Grillons). Ces groupes ont l'avantage d'être bien connus et sont représentatifs du type et de l'état du milieu qu'ils occupent, ce qui permet alors d'évaluer la valeur patrimoniale du site.» (Rainette 2013, 2017)

Ces citations nous amènent opportunément à répondre à notre première question. Les éléments synthétisés sur les papillons diurnes, les Odonates et les Orthoptères dans les trois parties de nos résultats ne vont pas dans le sens d'une retranscription systématiquement fidèle de l'ensemble des Insectes et autres Arthropodes et de leurs enjeux de conservation, ni d'être toujours représentatifs des milieux où vivent des Arthropodes sténotopes pour un site donné. En effet, ils occupent majoritairement les milieux dits ouverts, soit en particulier les prairies, pelouses et friches sèches ou humides (toutes incluses dans le type E d'EUNIS), plus secondairement les landes, fourrés, garrigues et toundras $(\mathrm{F})$, les tourbières hautes et bas-marais (D) ou bien sûr les milieux dulcicoles (C) pour les Libellules. On y trouve donc logiquement l'essentiel de leur diversité et de leurs enjeux de conservation, dont une majeure partie des 45 espèces à enjeux forts ou majeurs que nous avons définies pour ces trois groupes. Cette répartition écologique est d'ailleurs soutenue par plusieurs auteurs. D'après WallisDeVries \& Van Swaay (2009) et Warren et al. (2021), en Europe, les milieux prairiaux divers rassemblent de loin la plus grande richesse spécifique pour les Rhopalocères, suivis par les fourrés, landes et autres milieux arbustifs. Ils précisent que $88 \%$ des espèces européennes occupent les milieux prairiaux dans au moins un pays. Hochkirch et al. (2016) mentionnent que $74 \%$ des Orthoptères vivent dans les milieux ouverts, et que parmi celles considérées comme forestières, la plupart sont inféodées aux lisières, aux clairières et aux forêts claires.

En France, très peu d'espèces de ces groupes sont spécialistes des milieux littoraux tels que les plages et leurs annexes (e.g., schorres, dunes), et quasiment aucune des milieux souterrains (grottes, avens, MSS). Les sous-bois denses à strate herbacée peu développée sont également très peu prisés, 
puisque parmi les espèces considérées comme forestières, la plupart sont davantage liées aux clairières, aux lisières ou aux bois clairs (Hochkirch et al. 2016; Warren et al. 2021). Il en est d'ailleurs de même pour Cerambyx cerdo, un des deux Coléoptères à enjeux réglementaires les plus couramment rencontrés dans les études d'impact: il affectionne davantage les gros arbres situés en contexte relativement ensoleillé, ouvert à semi-ouvert (e.g., arbres isolés, lisière forestière, haie bocagère, bois clair), que ceux en forêt fermée, même si cette tendance s'atténue vers le Sud (Buse et al. 2007; DREAL Nouvelle-Aquitaine \& Société Entomologique du Limousin 2014; Brustel et al. 2019; Platek et al. 2019). Quant à Lucanus cervus, il est trop largement répandu et peu exigeant en France pour pouvoir être discriminant vis-à-vis d'un site à préserver (Brustel et al. 2019).

Certains Coléoptères protégés plus rares comme Osmoderma eremita (Scopoli, 1763) privilégient les mêmes contextes que ceux décrits ci-dessus pour Cerambyx cerdo; de même que dans une moindre mesure Rosalia alpina, cette dernière se trouvant tout de même davantage en hêtraie (Dubois 2009; Brustel et al. 2019). Quelques autres Coléoptères à statut réglementaire vivent davantage dans les massifs forestiers plus vastes et denses, de feuillus (e.g., Limoniscus violaceus (P. W. J. Müller, 1821)) ou de coniferes (e.g., Rhysodes sulcatus) (Brustel et al. 2019). Dans l'ensemble, les espèces d'Arthropodes forestiers prises en compte jusqu'ici, ainsi que les possibilités de détection (certains des Coléoptères ci-dessus étant fort rares et difficiles à détecter), restent minoritaires pour le type $\mathrm{G}$ dans les évaluations actuelles, en particulier pour les habitats forestiers fermés.

Ajoutons que si de rares espèces de Coléoptères protégés se trouvent davantage au sol (e.g., les Carabus protégés tels que C. solieri Dejean, 1826), la principale composante fonctionnelle ciblée par les quelques Coléoptères réglementaires est la faune saproxylophage des arbres dépérissants, morts ou à cavités. La faible représentation des espèces litiéricoles, lapidicoles, fissuricoles ou de celles vivant dans l'horizon supérieur du sol (hémi-édaphiques) est d'ailleurs aussi prégnante chez les Rhopalocères, Odonates et Orthoptères. Seuls les derniers comportent quelques espèces errantes s'abritant sous les pierres (e.g., Grillons comme Eugryllodes pipiens (Dufour, 1820) ou ceux du genre Pseudomogoplistes). En termes de fonctions écologiques, les prédateurs du sol, les nécrophages, les coprophages et les détritivores ne sont quasiment pas représentés dans les listes réglementaires.

Au niveau écologique, on peut donc affirmer que les papillons de jour, les Odonates et les Orthoptères sont de bons représentants des types milieux ouverts ( $\mathrm{E}$ et $\mathrm{F}$ d'EUNIS) et des milieux dulcicoles (C) ; plus secondairement des tourbières et des marais (D). Ils mettent bien en exergue les enjeux entomologiques dans ces habitats et plus largement leur bon état de conservation (Maciejewski et al. 2015). Ce sont de bons outils pour la restauration de ces milieux (Coulmin et al. 2020). Plus accessoirement, les Coléoptères à enjeux réglementaires les plus couramment trouvés (Cerambyx cerdo, Lucanus cervus) mettent en avant les «alignements d'arbres» (EUNIS G5.1).
A contrario, ils ne sont que très peu représentatifs des enjeux des Arthropodes terrestres dans les autres habitats, puisqu'ils n'y présentent que peu d'espèces écologiquement spécialisées en forêt fermée pour le type $\mathrm{G}$ et de cavernicoles pour le type $\mathrm{H} 1$ ( $\mathrm{H} 1$ = «grottes, systèmes de grottes, passages et plans d'eau souterrains terrestres»). Ils n'ont quasiment pas de spécialistes pour les milieux marins (A) et les milieux côtiers (B). Ils offrent une vision intéressante en termes de strates d'habitats (strates herbacée, arbustive, arborée) mais incomplète au niveau du sol et de ses micro-habitats. Enfin, s'il est indéniable qu'ils montrent de nombreuses particularités sur le plan biogéographique et sur celui de la responsabilité nationale (e.g., diversité élevée par rapport à de nombreux autres pays européens, bastion des populations pour certaines espèces menacées, nombreuses espèces en limite d'aire, présence de [sub]endémiques), deux d'entre eux (papillons diurnes, Odonates) appartiennent pourtant aux Arthropodes ayant les moins fort taux d'endémisme en France. De ce fait, ils ne peuvent refléter le juste niveau de la responsabilité française en matière de conservation des Arthropodes terrestres, a fortiori en observant que 3,2\% des espèces (faune et flore confondues) sont endémiques de France métropolitaine (Gargominy et al. 2020a) et que les Rhopalocères et Odonates ne comportent aucune espèce strictement endémique en métropole.

Mentionnons tout de même que la protection de deux sous-genres d'Aphaenops et du genre Trichaphaenops concerne 50 espèces, souvent des (sub)endémiques très localisées géographiquement, pour le type $\mathrm{H} 1$. Elles apparaissent très rarement trouvées lors d'études d'impact, les grottes faisant peu l'objet d'aménagements.

Ainsi, on ne peut généraliser sur le fait que les groupes actuellement utilisés dans les études d'impacts soient représentatifs de l'ensemble des Arthropodes terrestres, ni de leurs enjeux de conservation, ni des enjeux associés à certains habitats. Pour ce faire, il faudrait compléter les inventaires actuellement menés dans les études d'impact avec d'autres groupes ayant l'essentiel de leurs spécialisations écologiques et de leurs enjeux dans les habitats A (milieux marins, plus spécifiquement: niveau médiolittoral supérieur des plages; schorres, sansouïres), B (milieux côtiers: niveau supralittoral des plages; dunes), $\mathrm{G}$ (forêts) et $\mathrm{H}$ (habitats sans végétation); et si possible dans différents micro-habitats au sein de ces milieux.

\section{QU'APPORTERAIT L'AJOUT DE SIX AUTRES GROUPES}

D'ARTHROPODES POUR LA QUALITÉ DES DIAGNOSTICS DANS LES ÉTUDES D'IMPACT?

\section{Un cas remarquable: les plages maritimes et leurs milieux} annexes (schorres, dunes) ( $A$ et $B$ d'EUNIS)

À l'issue de notre sélection pour les six groupes évalués ici, a fortiori en tenant compte de son étroitesse et de sa localisation sur le trait de côte, cet ensemble d'habitats affiche l'accroissement le plus notable du nombre d'espèces à enjeux. Les Chilopodes, les Isopodes terrestres, les Coléoptères Scarabaeoidea et Tenebrionidae, les Hétéroptères Pentatomoidea ajoutent 25 espèces sténoèces exclusives des plages et de leurs annexes; les deux tiers étant de niveau d'enjeu majeur. 
Ajoutons que les Chilopodes, les Isopodes terrestres et les Tenebrionidae incluent encore d'autres espèces halophiles ou psammo-halophiles (Soldati 1995, 2007; Soldati \& Soldati 2002, sous presse; Jaulin \& Soldati 2005; Iorio 2014; Séchet \& Noël 2015; Racine \& Iorio 2017, 2020 ; Iorio et al. 2020), ce qui porte le nombre des spécialistes des plages et milieux annexes à plus d'une soixantaine en France (Corse incluse). Rien que chez les Tenebrionidae, il y a au total 31 espèces psammo-halophiles spécialistes du littoral dans notre pays.

Ajoutons que les milieux littoraux viennent, en partie (dunes côtières et rivages sableux méditerranéens), de faire l'objet d'une Liste rouge nationale (UICN France 2020). Cette Liste rouge montre que sur les huit écosystèmes évalués, six apparaissent comme menacés (un EN et cinq VU) et un seul comme non menacé (le dernier étant classé en DD). Complétant cette Liste rouge nationale partielle, les Listes rouges européennes des habitats terrestres et marins (Gubbay et al. 2016; Janssen et al. 2016) attribuent des statuts du côté de l'Atlantique et en complètent du côté de la Méditerranée. L'ensemble des habitats menacés ou quasi menacés abrite quatre cinquièmes des espèces littorales retenues dans nos listes, dont celles à enjeu majeur (Tableau 5).

$\mathrm{Si}$ on cumule les menaces sur les espèces de nos listes, celles formalisées par les Listes rouges et supplémentées par d'autres références littorales sur la pression concernant leurs habitats et leurs micro-habitats (Daligaux 2003; DREAL PACA 2013; Daeden 2015; Otero et al. 2018; Chauvin 2020), il est évident qu'une attention toute particulière doit être portée sur les Arthropodes spécialisés des plages et de leurs annexes (Fig. 20).

Il faut noter qu'une grande partie des habitats littoraux est ciblée par la Directive habitats-faune-flore et ils sont plutôt bien représentés dans le réseau Natura 2000 qui en découle. Ils sont également protégés dans le cadre des acquisitions foncières $\mathrm{du}$ "Conservatoire de l'espace littoral et des rivages lacustres » : à ce jour, 13 \% du linéaire côtier est préservé par ce conservatoire (http://www.conservatoire-du-littoral.fr/3-leconservatoire.htm, dernière consultation le 15 février 2021). Un raisonnement théorique pourrait conclure que ce cumul devrait permettre, par effet «parapluie», de préserver l'essentiel des Arthropodes de ces milieux. Cependant, la pratique le contredit sensiblement, en raison de l'absence de couverture de tous les sites à enjeux pour les Arthropodes, d'une gestion ne les prenant pas en compte et d'une poursuite des pressions (nettoyage des laisses de mer, artificialisation, fréquentation, nouveaux aménagements), que même les espaces protégés ont du mal à contre-balancer.

\section{Autres éléments de synthèse générale et par type d'habitat}

Jusqu'à présent, en dehors de quelques espèces réglementées de Coléoptères, ces six groupes n'ont presque jamais été pris en compte dans les études d'impact. Bien évidemment, les connaissances sur la plupart d'entre eux restent très largement inférieures à celles actuellement disponibles sur les Lépidoptères diurnes, les Odonates et les Orthoptères, sans parler des Vertébrés ou de la flore vasculaire (Touroult 2014). On peut y voir un effet circulaire de la réglementation: les espèces les mieux connues ont été plus facilement mises dans les premières listes de protection, ce qui en retour a suscité de nouvelles études, qui ont augmenté les données disponibles, permettant de faire des Listes rouges, qui elles-mêmes facilitent la prise en compte dans les études réglementaires. Ce cycle ne serait pas un problème s'il ne conduisait en parallèle d'autres groupes à rester négligés, faute de l'investissement minimal permettant de les prendre en compte dans les politiques de conservation.

Pour autant, les connaissances en matière de distribution des espèces de ces six groupes se sont considérablement améliorées depuis 2000 et plus encore depuis 2010. Nous en voulons pour preuve une comparaison du nombre moyen de données/ espèce de chacun de ces groupes en France $v$ le nombre de données ayant très récemment servi à l'établissement d'une Liste rouge de 219 Scarabaeoidea méditerranéens (Numa et al. 2020). Pour cette dernière, un ratio moyen de 109 données/ espèce dans l'ensemble de la Région méditerranéenne (Europe, Proche-Orient, Afrique du Nord) a permis d'attribuer un statut formel (CR, EN, VU, NT ou LC) à $63 \%$ des espèces évaluées, tandis que $37 \%$ sont tombées en «données insuffisantes" (DD); des chiffres encourageants sur la capacité d'évaluation. Or, parmi les groupes que nous avons évalués pour leurs enjeux de conservation dans le présent article, le ratio le plus faible est de 150 données/espèces. Ainsi, même si leur connaissance reste évidemment perfectible et que des régions sont moins bien connues que d'autres, il y a suffisamment de recul sur ces groupes pour ne plus les négliger. Cette observation prend d'autant plus de sens quand on observe le nombre de données qui était disponible sur les Orthoptères au début des années 2000 et la dynamique qui s'est créée sur ces Insectes, y compris au sein des bureaux d'études.

Comme l'illustre notre sélection de 90 espèces à enjeux forts ou majeurs et plus globalement leur écologie et leur biogéographie, ces six groupes rassemblent des enjeux de conservation importants, avec des exigences écologiques majoritairement différentes de celles des Lépidoptères diurnes, Odonates et Orthoptères. Leur rôle fonctionnel dans les écosystèmes est aussi distinct: régulation des invertébrés du sol et de la litière (Chilopodes), recyclage des matières organiques au sol (Isopodes), du bois mort (Longicornes xylophages) et des excréments (Scarabaeidae coprophages), opophages ou prédateurs sur la végétation (Pentatomoidea). Enfin, les Chilopodes, les Isopodes terrestres et les Tenebrionidae font davantage ressortir l'endémisme au sein du phylum des Arthropodes, dépassant allègrement la moyenne métropolitaine (Gargominy et al. 2020a). Bien qu'étant loin de refléter les multiples facettes de l'endémisme, la prise en compte de ces espèces contribuerait à améliorer cet aspect parfois négligé au profit des seules Listes rouges.

Quelques exemples d'habitats évalués dans les études d'impact et revus avec ces groupes montrent qu'ils pourraient apporter des compléments intéressants, pour ne pas dire indispensables dans le cas des milieux littoraux. Pour ces derniers, on se rend compte que même si les types A et $\mathrm{B}$ sont peu représentés dans les 50 études examinées, cela ne peut expliquer l'absence d'enjeux dans ceux-ci s'ils sont relativement préservés. Cet aspect complémentaire ressort 
TABLEAU 5. - Habitats littoraux en déclin, abritant des Arthropodes à enjeux majeurs chez les neuf groupes étudiés. Abréviations: France, Liste rouge nationale pour les Odonates (UICN France et al. 2016), proposition selon la méthode Liste rouge de l'UICN pour les Chilopodes (Annexe 3) et Liste rouge non UICN de Sardet \& Defaut (2004) pour les Orthoptères (DD, données insuffisantes; EN, en danger d'extinction; LR1, proche de l'extinction; NT, quasi menacé; VU, vulnérable). Pour les autres groupes, mention dans colonne «France » du déclin selon correspondance avec ce critère dans notre matrice de choix (Tableau 2); LR méd., Liste rouge méditerranéenne des Scarabaeidae (Numa et al. 2020); LRE, Liste rouge européenne (habitats: Gubbay et al. 2016; Janssen et al. 2016; Soldo 2016; Odonates: Kalkman et al. 2010; Orthoptères: Hochkirch et al. 2016); LRN, Liste rouge nationale des habitats (UICN France 2020).

\begin{tabular}{|c|c|c|c|c|c|}
\hline Habitat & LRN & LRE & Espèces de niveau majeur & France & $\begin{array}{l}\text { LR méd./ } \\
\text { LRE }\end{array}$ \\
\hline $\begin{array}{l}\text { A2.13 (marge haute) - Communautés des } \\
\text { sédiments grossiers médiolittoraux } \\
\text { méditerranéens (aussi banquettes de posidonies } \\
\text { en A.2131) }\end{array}$ & - & $\begin{array}{l}\text { DD } \\
\text { (supposé } \\
\text { en déclin) }\end{array}$ & $\begin{array}{l}\text { Chilopode Henia bicarinata } \\
\text { Chilopode Tuoba poseidonis }\end{array}$ & $\begin{array}{l}\text { EN } \\
\text { EN }\end{array}$ & - \\
\hline $\begin{array}{l}\text { A2.25 (marge haute) - Communautés des sables } \\
\text { médiolittoraux méditerranéens (plages à } \\
\text { sédiments allant du sable fin aux graviers) }\end{array}$ & - & VU & $\begin{array}{l}\text { Chilopode Henia bicarinata } \\
\text { Chilopode Tuoba poseidonis }\end{array}$ & $\begin{array}{l}\text { EN } \\
\text { EN }\end{array}$ & - \\
\hline $\begin{array}{l}\text { A2.5c - Marais salés côtiers atlantiques } \\
\text { (= schorres) }\end{array}$ & - & VU & $\begin{array}{l}\text { Chilopode Schendyla monodi } \\
\text { Orthoptère Epacromius tergestinus } \\
\quad \text { tergestinus }\end{array}$ & $\begin{array}{l}\text { VU } \\
\text { PR1 }\end{array}$ & $\overline{E N}$ \\
\hline A2.5d - Marais salés côtiers méditerranéens & - & NT & $\begin{array}{l}\text { Odonate Lestes macrostigma } \\
\text { Coléoptère Gonocephalum calcaripes }\end{array}$ & $\begin{array}{l}\text { EN } \\
\text { Déclin }\end{array}$ & $\begin{array}{l}\text { VU } \\
-\end{array}$ \\
\hline $\begin{array}{l}\text { B1.1a - Plages sableuses atlantiques (sédiments } \\
\text { allant du sable fin aux graviers; niveau des } \\
\text { laisses de mer) }\end{array}$ & - & VU & Coléoptère Calicnemis obesa & Déclin & - \\
\hline $\begin{array}{l}\text { B1.1b - Plages sableuses méditerranéennes } \\
\text { (sédiments allant du sable fin aux graviers; } \\
\text { niveau des laisses de mer) }\end{array}$ & VU & NT & $\begin{array}{l}\text { Chilopode Henia bicarinata (partie basse } \\
\text { de B1.1b) } \\
\text { Chilopode Tuoba poseidonis (partie } \\
\text { basse de B1.1b) } \\
\text { Isopode Trichoniscus halophilus } \\
\text { Coléoptère Calicnemis latreillii } \\
\text { Coléoptère Phaleria acuminata }\end{array}$ & $\begin{array}{l}\text { EN } \\
\text { EN } \\
\text { Déclin } \\
\text { Déclin } \\
\text { Déclin }\end{array}$ & $\begin{array}{l}- \\
- \\
- \\
- \\
-\end{array}$ \\
\hline $\begin{array}{l}\text { B1.3a - Dunes côtières mobiles atlantiques (dunes } \\
\text { blanches) }\end{array}$ & - & NT & Hétéroptère Phimodera humeralis & Déclin & - \\
\hline $\begin{array}{l}\text { B1.3b - Dunes côtières mobiles méditerranéennes } \\
\text { (dunes blanches) }\end{array}$ & EN & VU & $\begin{array}{l}\text { Coléoptère Calicnemis latreillii } \\
\text { Coléoptère Scarabaeus semipunctatus } \\
\text { Coléoptère Thorectes sardous } \\
\text { Coléoptère Heliopates littoralis } \\
\text { Coléoptère Phaleria acuminata } \\
\text { Coléoptère Pimelia muricata cyrnensis } \\
\text { Coléoptère Pseudoseriscius pruinosus } \\
\text { Hétéroptère Cephalocteus scarabaeoides }\end{array}$ & $\begin{array}{l}\text { Déclin } \\
\text { Déclin } \\
\text { Déclin } \\
\text { Déclin } \\
\text { Déclin } \\
\text { Déclin } \\
\text { Déclin } \\
\text { Très fort } \\
\text { déclin }\end{array}$ & $\begin{array}{l}- \\
\text { VU } \\
\text { EN } \\
- \\
- \\
- \\
-\end{array}$ \\
\hline $\begin{array}{l}\text { B1.4a - Prairies dunaires côtières atlantiques } \\
\text { (dunes grises) }\end{array}$ & - & VU & $\begin{array}{l}\text { Coléoptère Thorectes sericeus } \\
\text { Hétéroptère Phimodera humeralis (partie } \\
\text { basse de } \mathrm{B} 1.4 \mathrm{a} \text { ) }\end{array}$ & $\begin{array}{l}? \\
\text { Déclin }\end{array}$ & - \\
\hline $\begin{array}{l}\text { B1.4b - Prairies dunaires côtières } \\
\text { méditerranéennes (dunes grises) }\end{array}$ & VU & EN & $\begin{array}{l}\text { Coléoptère Scarabaeus semipunctatus } \\
\text { (partie basse de B1.4b) } \\
\text { Coléoptère Thorectes sardous } \\
\text { Coléoptère Heliopates littoralis } \\
\text { Coléoptère Pimelia muricata cyrnensis }\end{array}$ & $\begin{array}{l}\text { Déclin } \\
\text { Déclin } \\
\text { Déclin } \\
\text { Déclin }\end{array}$ & $\begin{array}{l}\text { VU } \\
\text { EN } \\
- \\
-\end{array}$ \\
\hline
\end{tabular}

d'autant plus que dans ces exemples, les Lépidoptères diurnes, les Odonates, les Orthoptères n’avaient révélé aucun enjeu particulier, ce qui est logique, compte-tenu du fait qu'ils n'ont guère d'espèces spécialisées sur ces milieux. Il est utile de constater que ces cas de figure, tout comme certainement de nombreux autres concernant les mêmes habitats, peuvent contenir des enjeux considérables. A fortiori sachant que pour tous les groupes, il a fallu faire des choix, parfois délicats, pour ne garder que 15 espèces à enjeux importants. Ainsi, d'autres espèces menacées ou encore nombre d'endémiques n’y figurent pas alors qu'elles pourraient aussi être considérées comme des enjeux notables.
Les milieux forestiers fermés (massifs à canopée recouvrante) (G dans EUNIS) dévoilent aussi nombre d'enjeux particuliers chez les Chilopodes, les Isopodes terrestres, les Longicornes et les Scarabaeoidea. Les deux derniers sont aussi utiles pour renforcer la prise en compte de l'habitat G5.1 (arbres isolés de parc, bocage, alignement d'arbres). Soulignons que plusieurs écosystèmes forestiers apparaissent aussi menacés (UICN France 2016, 2018b) : les châtaigneraies et subéraies méditerranéennes, les hêtraies, les pessières et les sapinières jugées VU; les chênaies pubescentes méditerranéennes jugées "quasi menacées». Plusieurs des espèces forestières à enjeux que nous avons sélectionnées vivent majoritairement, voire 


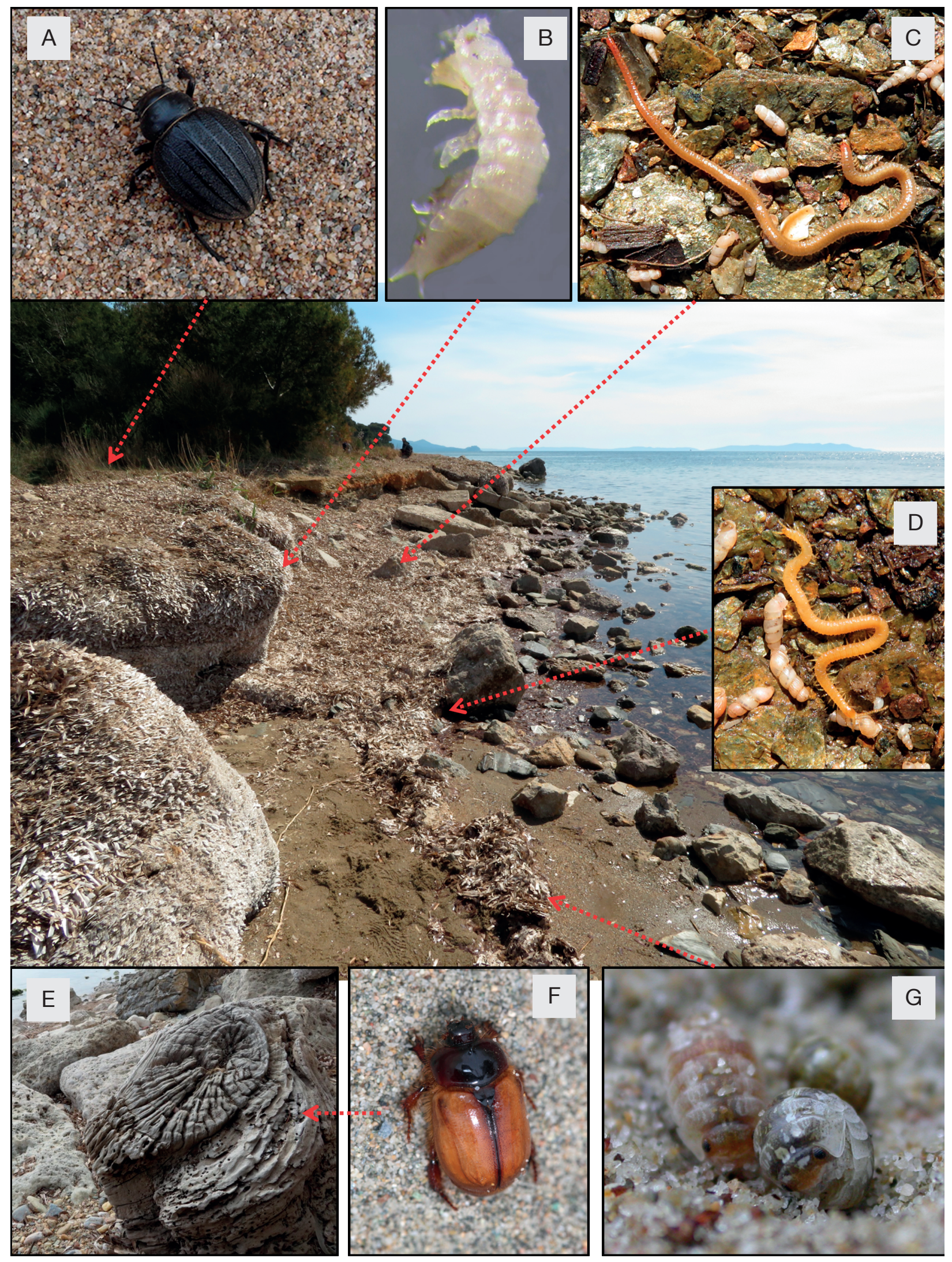

FIG. 20. - Exemple d'une plage varoise relativement préservée et de plusieurs espèces à enjeux élevés qu'elle abrite. A, Pimelia muricata cyrnensis F. Soldati \& Rougon, 2017, qui affectionne l'étroite bande d'ammophilaie en arrière-plan; B, Trichoniscus halophilus Vandel, 1951 des banquettes de posidonies; C, Henia bicarinata (Meinert, 1870), sous les pierres en contact étroit avec le substrat ou les posidonies; D, Tuoba poseidonis (Verhoeff, 1901) comme H. bicarinata, mais se trouvant souvent plus bas sur le bord de mer; E, bois flotté vu un peu plus loin, propice à Calicnemis latreillii Laporte de Castelnau, 1832 (F); G, Tylos europaeus Arcangeli, 1938 sous les pierres ou les posidonies. Les échelles sont volontairement omises. Crédits photos: A, F. Soldati; B \& G, F. Noël; C, D, E et vue de la plage, E. lorio; F, H. Bouyon. 
exclusivement dans l'un ou l'autre de ces habitats: Chilopodes: Eupolybothrus tridentinus (Fanzago, 1874), Lithobius dentatus et $L$. pelidnus; Isopodes: Tiroloscia esterelana (Verhoeff, 1918); Coléoptères: Necydalis major Linnaeus, 1758, N. ulmi Chevrolat, 1838, Prinobius myardi Mulsant, 1842, Tragosoma depsarium (Linnaeus, 1767), Eupotosia mirifica (Mulsant, 1842) et Ceruchus chrysomelinus. N. ulmi et $E$. mirifica sont en plus jugés VU dans la Liste rouge européenne (Cálix et al. 2018) et E. tridentinus et T. esterelana ont une répartition très limitée en France. Comme pour les milieux littoraux, les habitats forestiers sont bien représentés dans les habitats d'intérêt communautaire du réseau Natura 2000, à l'exception notable des chênaies pubescentes. Ils abritent également des espèces vertébrées à enjeux de conservation reconnues (Oiseaux, Mammiferes), dont on pourrait penser qu'elles puissent servir de "parapluie" pour la conservation des Arthropodes. Cependant, en pratique, la protection des habitats naturels et de Vertébrés relativement mobiles, voire à très larges capacités de dispersion pour certains (Oiseaux, Chiroptères), ne ciblent pas suffisamment les sites relictuels (forêt anciennes et matures), ni les espèces endémiques, ni les espèces à faibles capacités de dispersion d'Arthropodes.

Le cas du type H d'EUNIS (milieux dépourvus de végétation) est aussi intéressant. D'un côté il renferme d'assez nombreuses espèces troglobies ou eutroglophiles à enjeux très marquants dans les grottes $(\mathrm{H} 1)$, pour la plupart endémiques de France, au sein des Chilopodes, des Isopodes terrestres et des Tenebrionidae (en plus des Coléoptères protégés Aphaenops et Trichaphaenops); dont de nombreux cas non inclus dans les listes de l'Annexe 2 (e.g., Soldati 2007; Iorio 2014; Séchet \& Noël 2015; Iorio \& Geoffroy 2019). De l'autre, les milieux à végétation (très) clairsemée abrite des Pentatomoidea remarquables ( $\mathrm{H} 2$ et $\mathrm{H} 3$ ). Ces milieux subissent une pression relativement faible et sont en meilleur état de conservation que les autres habitats d'intérêt communautaire (Bensettiti \& Puissauve 2015). Pour les grottes, leur faune serait relativement stable et résiliente (Faille et al. 2014). Cependant, des cas d'altération anthropique de stations, pouvant causer la disparition d'endémiques cavernicoles, sont connus (Silva \& Ferreira 2015; Borges et al. 2019). Ainsi, les espèces listées ici auraient plutôt un rôle préventif, au cas où des aménagements viendraient à être réalisés sur ces milieux jusqu'ici relativement plus préservés.

En revanche, les types $\mathrm{E}$ (milieux prairiaux) et $\mathrm{F}$ (landes, fourrés, garrigues) comportent deux à trois fois moins d'espèces à enjeux considérables pour ces six groupes que pour les papillons diurnes, Odonates et Orthoptères cumulés; et encore nettement moins pour certains de ces groupes en les confrontant indépendamment aux papillons ou aux Orthoptères. Les types $\mathrm{C}$ et $\mathrm{D}$ sont aussi très minoritaires chez les six "nouveaux», n’ayant pas de cycle larvaire aquatique contrairement aux Odonates.

En bref, une vision d'ensemble fondée sur davantage de groupes tend logiquement vers beaucoup plus de complétude à défaut d'être exhaustive et pourrait combler certaines des lacunes majeures pour la conservation des invertébrés (Cardoso et al. 2011). Un panel nettement plus représentatif sur le plan taxinomique (plus d'ordres représentés), biogéographique (plus d'espèces endémiques et d'espèces localisées) et écologique (habitats et fonctions) pourrait donc être dressé dans les études d'impact. Il contribuerait à une meilleure évaluation des enjeux pour les habitats A, B, G et $\mathrm{H}$. Ceci s'inscrit dans la logique des groupes préconisés pour renforcer les inventaires nationaux (Touroult et al. 2015), notamment pour mieux représenter les espèces à répartition restreinte. Ces dernières sont déterminantes pour renforcer efficacement la conservation «spatiale» de la biodiversité (Manne \& Williams 2003).

\section{La fonction de "parapluie» et les espèces protégées}

D'après Roberge \& Angelstam (2004), une espèce "parapluie» est "une espèce dont la conservation devrait conférer une protection à un grand nombre d'espèces naturelles concomitantes ». Ces auteurs donnent aussi des conseils sur la manière de retenir ces espèces, dont notamment:

- la nécessité d'une approche multi-espèces, donc par de multiples "parapluie», comme moyen d'élargir la portée de la protection;

- les espèces doivent couvrir les principaux types d'écosystèmes d'une région donnée;

- pour chaque type d'habitat, les espèces les plus sensibles en termes de ressources, d'exigences, de connectivité et de processus naturels devraient être choisies;

- selon le contexte d'application, le cumul des espèces peut représenter une gamme d'écorégions ou d'environnements des habitats et micro-habitats localisés.

Comme les espèces protégées au niveau national impliquent légalement une prise en compte de leurs populations et le plus souvent de leurs habitats dans les études d'impact (Arrêté du 23 avril 2007: article 2), elles pourraient être des "parapluies réglementaires" si elles répondaient systématiquement aux critères de Roberge \& Angelstam (2004). En effet, elles constitueraient la première "barrière" face à une volonté d'aménagement menaçant des milieux naturels, ceux-ci abritant de nombreuses autres espèces.

Cependant, deux macrohabitats du niveau 1 de la typologie d'EUNIS sont dépourvus d'espèces d'Arthropodes protégées, i.e. les types $A$ et $B$ : la barrière réglementaire est donc véritablement absente pour eux. Il n'y a aucune obligation pour un porteur de projet de prendre en considération les éventuelles espèces vraiment sensibles et menacées de ces habitats, potentiellement nombreuses comme nous l'avons vu précédemment. Ceci constitue une sérieuse lacune. On peut étendre cette remarque aux macrohabitats vastes et très diversifiés comme les milieux forestiers $(G)$, trop faiblement représentés par les espèces protégées actuelles; ou encore au type $\mathrm{H}$, notamment aux milieux souterrains $(\mathrm{H} 1)$ avec beaucoup d'endémiques induisant une responsabilité nationale élevée. Les listes d'espèces actuellement protégées sont donc bien lacunaires.

Globalement, il nous semble que les Arthropodes sont loin d'être complètement couverts par les autres éléments réglementés de la biodiversité et qu'ils méritent une attention particulière, compte-tenu de leurs traits biologiques. Ainsi leurs faibles capacités de dispersion, leur forte spécialisation à un habitat ou micro-habitat, leurs fonctions écologiques et le fort endémisme pour certains, impliquent qu'ils peuvent ne pas 
être préservés par des démarches sur des espèces plus mobiles, ou sur un habitat au sens large (Leather 2013). Le besoin de compléter les listes de protection des Arthropodes est largement reconnu au niveau européen, que ce soit globalement (Cardoso et al. 2012) ou pour un groupe fonctionnel particulier comme les coprophages (Numa et al. 2020).

A contrario, on peut s'interroger sur la nécessité de maintenir une protection nationale pour quelques espèces qui ne répondent à aucune des prérogatives du troisième alinéa de Roberge \& Angelstam (2004) sur leur sensibilité. Un cas flagrant est le papillon Zerynthia polyxena. D'une part, cette espèce relativement fréquente n'est pas menacée à court ou moyen terme. D'autre part, son caractère eurytope, sa facilité d'adaptation et celle de sa plante-hôte principale à des milieux "naturels", fragmentés et situés non rarement en contexte relativement anthropisé, du moment qu'ils présentent quelques caractéristiques abiotiques minimales (humidité et/ ou ombrage), en font un piètre "parapluie». Pour beaucoup, les espèces sténotopes et menacées de milieux mésophiles à hygrophiles, forestières ou de milieux ouverts, ne peuvent se maintenir dans des habitats aussi fragmentés et/ou anthropisés que ceux occupés par la Diane. C'est notamment le cas de la plupart des 135 espèces à enjeux forts ou majeurs définies dans cet article. Aussi est-il est surprenant de voir que $Z$. polyxena a encore été intégrée récemment dans un plan national d'actions (Houard \& Jaulin 2018); la raison étant probablement son statut réglementaire. La nécessité d'une révision apparaît aussi pour d'autres auteurs, puisque Hok Yau Tang \& Visconti (2020) font remarquer qu'une grande partie des espèces européennes d'Odonates les plus menacées ne sont pas protégées par la Directive Habitats. Ce cas d'espèces fortement menacées et non protégées concerne aussi plusieurs Odonates, Rhopalocères et Orthoptères en France, comme l'attestent certaines des 15 espèces à enjeux forts et majeurs sélectionnées pour eux mais aussi d'autres espèces des Listes rouges nationales (UICN France \& MNHN 2012; UICN France et al. 2016). Heureusement, pour les Odonates et les Rhopalocères, le nombre relativement élevé d'espèces protégées induit un meilleur effet "parapluie réglementaire», même s'il est assurément perfectible.

Au moins pour les milieux marins et côtiers (types A et B d'EUNIS), l'absence d'espèces protégées pourrait être comblée par une sélection d'espèces parmi les plus aisées à identifier et à rechercher de nos listes (Tableau 6), en fonction des critères de Roberge \& Angelstam (2004). Cette démarche permettrait de couvrir entièrement les milieux et les micro-habitats liés aux plages et arrières-plages, de l'estran sableux ou pierreux aux dunes, ainsi que les sansouires et mares saumâtres des côtes françaises.

Bien entendu, et comme cela se fait déjà pour les groupes couramment étudiés dans les études d'impact, il est indispensable de ne pas limiter le diagnostic aux seules espèces protégées, parce qu'elles ne peuvent offrir à elles seules une vision optimale des groupes concernés; d'où le concept global d'espèces à «enjeux de conservation ». De plus, la prise en compte des seules espèces protégées peut parfois amener la destruction d'habitats et/ou micro-habitats primordiaux pour d'autres groupes. Ainsi, la destruction de zones boisées anciennes, parfois compensée par des replantations d'arbres jeunes, ou encore le déplacement des troncs, support de vie des espèces protégées (Cerambyx cerdo, Osmoderma eremita), ne prennent pas en compte la faune du sol et de la litière, pouvant elle aussi être menacée. Roberge \& Angelstam (2004) mentionnent que «le concept d'espèce parapluie n'est pas une panacée et que même un système parapluie très sophistiqué ne pourrait probablement pas garantir la protection de toutes les espèces», ce qui renforce la nécessité d'une approche multiespèces et même multi-groupes dans les évaluations.

Il en est d'ailleurs de même en axant la réflexion sur nos listes des 15 espèces à enjeux forts et majeurs pour chacun des groupes retenus. D'autres espèces peuvent évidemment aussi avoir d'importants enjeux, a fortiori lors d'un focus sur des enjeux de conservation au niveau local ou régional. Nos choix ont simplement l'objectif de fournir un échantillon représentatif d'espèces à enjeux de conservation aussi remarquables que pratiques, pour aider à une meilleure prise en compte des Arthropodes continentaux en fonction des habitats naturels concernés.

Pour s'assurer du succès de la mise en œuvre de cette approche multi-taxons, un effort particulier doit également être entrepris sur la formation des ingénieurs écologues et des naturalistes qui réalisent les études. Force est de constater aujourd'hui que les formations en taxinomie et identification des Arthropodes sont très limitées, si l'on excepte les Rhopalocères, Odonates et Orthoptères.

\section{CONCLUSION}

Le présent article permet de dresser un premier bilan sur l'approche des Arthropodes continentaux au sein des volets naturels d'études d'impact. Le triptyque constitué par les papillons diurnes, Odonates et Orthoptères a été largement priorisé jusqu'ici, en partie en raison des protections réglementaires attribuées à certaines espèces. Le constat est que depuis d'assez nombreuses années, la prise en compte s'est clairement élargie à l'ensemble de ce triptyque et non uniquement aux espèces réglementaires. De cette priorisation, il résulte que les enjeux inhérents à certains habitats naturels ont été bien soulignés jusqu'ici, notamment pour les milieux prairiaux (type E du niveau 1 d'EUNIS), les landes, fourrés et garrigues (F), et les milieux dulcicoles (C); plus secondairement pour les tourbières et les marais (D) et pour les arbres isolés et alignements d'arbres (G5.1). En revanche, on ne peut pas en dire autant pour les milieux marins (A), les milieux côtiers (B), les milieux sans végétation $(\mathrm{H})$ et les habitats forestiers fermés du type $\mathrm{G}$, qui s'avèrent particulièrement dépourvus d'espèces à enjeux notables chez les papillons diurnes, les Odonates et les Orthoptères. Pour ces derniers groupes d'habitats, il serait nécessaire d'en élargir l'étude à d'autres groupes d'Arthropodes terrestres pour remédier aux sous-évaluations des enjeux de conservation. La conservation de nombreuses espèces menacées en France, en particulier dans les milieux déjà soumis à forte pression anthropique, en dépend fortement. 
TABLEAU 6. - Propositions d'espèces menacées, complémentaires et à enjeux considérables qu'il serait utile de protéger au niveau national afin de couvrir correctement les habitats les plus démunis au niveau réglementaire. *, plusieurs espèces très proches existent en dehors de l'aire géographique précisée pour ces deux Scarabeidae.

\begin{tabular}{|c|c|c|}
\hline $\begin{array}{l}\text { Grands types d'habitats } \\
\text { selon la classification EUNIS }\end{array}$ & $\begin{array}{l}\text { Espèces d'Arthropodes continentaux } \\
\text { qu'il serait nécessaire de protéger }\end{array}$ & Rappel des habitats \\
\hline A - Habitats marins & $\begin{array}{l}\text { Chilopode Henia bicarinata } \\
\text { Chilopode Tuoba poseidonis } \\
\text { Isopodes Tylos spp. } \\
\text { Hétéroptère Brachynema cinctum } \\
\text { Odonate Lestes macrostigma } \\
\text { Criquet Epacromius tergestinus tergestinus }\end{array}$ & $\begin{array}{l}\text { Plages méditerranéennes en marge } \\
\text { médiolittorale supérieure } \\
\text { Plages méditerranéennes en marge } \\
\text { médiolittorale supérieure } \\
\text { Plages méditerranéennes en marge } \\
\text { médiolittorale supérieure } \\
\text { Sansouires et marais saumâtres méditerranéens } \\
\text { Mares saumâtres à Scirpe maritime et Jonc } \\
\text { maritime } \\
\text { Schorres de l'Atlantique }\end{array}$ \\
\hline B - Habitats côtiers & $\begin{array}{l}\text { Chilopode Henia bicarinata } \\
\text { Chilopode Tuoba poseidonis } \\
\text { Isopodes Tylos spp. } \\
\text { Isopode Armadillidium album } \\
\text { Coléoptère Scarabeidae Scarabaeus semipunctatus } \\
\text { Coléoptère Scarabeidae Calicnemis latreillii* } \\
\text { Coléoptère Scarabeidae Thorectes sericeus* } \\
\text { Coléoptère Tenebrionidae Heliopates littoralis } \\
\text { Coléoptère Tenebrionidae Pimelia muricata cyrnensis } \\
\text { Coléoptère Tenebrionidae Pseudoseriscius pruinosus } \\
\text { Hétéroptère Phimodera humeralis }\end{array}$ & $\begin{array}{l}\text { Plages méditerranéennes au niveau supralittoral } \\
\text { Plages méditerranéennes au niveau supralittoral } \\
\text { Plages méditerranéennes au niveau supralittoral } \\
\text { Plages de l'Atlantique et de la Manche au } \\
\text { niveau supralittoral, voire un peu au-dessus } \\
\text { Dunes blanches méditerranéennes } \\
\text { Plages méditerranéennes au niveau supralittoral } \\
\text { Dunes grises landaises } \\
\text { Dunes blanches et grises méditerranéennes } \\
\text { Dunes blanches et grises méditerranéennes } \\
\text { Dunes blanches méditerranéennes } \\
\text { Dunes blanches et partie basse des dunes } \\
\text { grises du littoral aquitain }\end{array}$ \\
\hline
\end{tabular}

Les six groupes proposés en complément (Chilopodes, Isopodes terrestres, Coléoptères Longicornes, Scarabaeoidea et Tenebrionidae, Hétéroptères Pentatomoidea) permettent de combler des lacunes pour les habitats négligés. Ils disposent pour ceux-ci d'espèces à enjeux de conservation élevés et bonnes "parapluies» pour d'autres d'identification délicate, mais partageant le même habitat. Associés aux Lépidoptères diurnes, Odonates et Orthoptères, ainsi qu'aux Coléoptères déjà dotés d'enjeux réglementaires, une meilleure approche et préservation des habitats évoqués plus haut pourrait être atteinte. Dans un grand nombre des études d'impact examinées, un expert différent intervient pour chaque groupe de Vertébrés (i.e. un ornithologue, un herpétologue-batrachologue, un mammalogue voire souvent un chiroptérologue). Pourquoi n'en serait-il pas ainsi, à terme, chez les Arthropodes terrestres qui représentent environ $85 \%$ des espèces animales décrites (Chevassus-au-Louis 2005) ? Pour couvrir les neuf groupes d'Arthropodes de cet article, il suffirait de former deux ou trois experts complémentaires pour être en mesure de traiter le volet "Insectes et autres Arthropodes», ce qui paraîtrait plus en adéquation avec la richesse de ce phylum, tout en restant pragmatique: il est bien sûr hors de question de pouvoir le cerner en intégralité.

Le lecteur gardera bien à l'esprit que la prise en compte des groupes proposés ne vise pas à refléter la totalité des enjeux impliquant les Arthropodes continentaux. D'autres groupes pourraient utilement compléter ceux traités dans le présent article, ne serait-ce qu'en se limitant à quelques familles peu diversifiées et pour lesquelles il existe des outils pratiques en français pour la reconnaissance des espèces (e.g., macro-hétérocères, petites familles d'Araignées). La sélection proposée est avant tout pragmatique et novatrice, surtout pour les habitats jusqu' ici négligés bien que riches en espèces à enjeux importants.

En guise de perspectives, et fort des arguments développés dans cet article, nous appelons de nos vœux:

- que les DREAL, les CSRPN, les associations naturalistes, les principaux pétitionnaires et les bureaux d'études prennent conscience des neuf groupes proposés et de leurs enjeux de conservation associés. Comme nous l'avons vu pour les «nouveaux " groupes, des formations existent, même si elles devraient être amplifiées, et divers ouvrages disponibles aident à leur identification et à cerner leur écologie. Des fiches pratiques pourraient même être proposées en ce sens (des exemples existent déjà pour certaines espèces des «nouveaux" groupes);

- qu'un statut de protection nationale soit envisagé pour toutes les espèces proposées (Tableau 6), dont les enjeux de conservation sont évidents. Il est cependant indispensable que cette protection soit fondée sur les habitats abritant ces taxons, et non sur les spécimens en tant que tels. Par le passé, la confusion entre protection au titre de la conservation biologique, et protection contre l'acte de collecte - et donc d'étude - a été particulièrement inadaptée aux Arthropodes (la sensibilité étant forte sur les perturbations d'habitats et de micro-habitats, mais très faible vis-à-vis des prélèvements raisonnés d'individus chez les espèces: la capture ne concernant, dans un habitat et site donné, qu'une infime partie de leur population locale);

- que ces mesures de protection soient aussi périodiquement révisées ou au moins complétées, avec un regard plus large que celui opéré jusqu'ici pour les Arthropodes et leurs habitats.

Enfin, une étude qui concernerait la prise en compte des Arthropodes continentaux au sein des Documents d'Objectifs (DOCOB) du réseau Natura 2000 serait également utileà réaliser. 


\section{Remerciements}

Nous sommes très reconnaissants envers les experts qui ont participé aux réflexions sur les espèces à enjeux de conservation, en nous donnant leurs précieux avis, conseils et suggestions: Thomas Barnouin, Jean-Pierre Boudot, Yoan Braud, Hervé Brustel, Thomas Cherpitel, Sylvain Delmas, Éric Drouet, Jean-Pierre Favretto, Ludovic Fuchs, Jean-Jacques Geoffroy, Nicolas Gouix, Karim Guerbaa, Arnaud Horellou, Guillaume Jacquemin, Bruno Mériguet, Antoine Racine, Sonia Richaud, Emmanuel Séchet, Marielle Tardy et Stéphane Vassel. Nous remercions aussi de nouveau Antoine Racine, ainsi qu'Arzhvaël Jeusset (OFB, PatriNat, chargé de mission "Liste rouge») et Lena Baraud (UICN, chargée de mission «Espèces menacées»), experts de la méthode Liste rouge de l'UICN, pour les heures passées sur l'évaluation des 15 Chilopodes selon cette méthode et les échanges très constructifs qui en ont émané. Marielle Tardy a aimablement apporté ses données de Diane pour élargir notre vision.

Merci aussi à Éric Texier (AER) et Alain Livory (Manche Nature) qui ont utilement complété nos informations sur Lithobius variegatus. Au groupe Myria-France, au GRETIA et à leurs membres, qui, par leurs apports de données, contribuent à accroître la dynamique sur les Myriapodes de France. À Cécile Bayle, collègue botaniste à ECOTER, pour les discussions très instructives sur la typologie EUNIS, ainsi qu'à Hervé Bouyon pour sa photo de Calicnemis latreillii et Océane Vellot (ECOTER) pour ses photos de Magicienne dentelée et d'Eurydema herbacea. Enfin, nous remercions les collègues qui nous ont donné des informations pour mieux cerner le nombre approximatif de données sur les papillons diurnes et les Orthoptères: Marie-Cécile Andrei-Ruiz (OCIC), Sylvain Lethuillier et Frédéric Morra (CBNFC-ORI); de même que les deux référés Nicolas Gouix et Brian Padilla pour leur relecture et leurs suggestions pour améliorer le manuscrit.

\section{RÉFÉRENCES}

AMYot C. J.-B. \& Audinet-SERVILle J.-G. 1843. — Histoire naturelle des Insectes. Librairie Encyclopédique de Roret, Paris, 676 p.

ARB ÎLE-DE-FRANCE 2020. - Atlas dynamique francilien des sauterelles, grillons, criquets. https://www.arb-idf.fr/article/atlasdynamique-francilien-des-sauterelles-grillons-criquets/, dernière consultation 2020.

ARChauX F., Bichaud M., Chatard P., FAUCheux F. \& LÉvêQue A. 2016. - Zygènes et papillons de jour du Loiret. Insectes 182: 15-20.

Aubin G., BenCE S., Richaud S., Maurel N. \& ManON P. 2019. Zerynthia polyxena (Denis \& Schiffermüller, 1775), in BENCE S. \& RICHAUD S. (éds), Atlas des papillons de jour et Zygènes de ProvenceAlpes-Côte d'Azur. Le Naturographe, Gap: 226-227.

Aukema B. 2010. - Phimodera humeralis na bijna 70 jaar weer in nederland waargenomen (heteroptera: scutelleridae). Nederlandse Faunistische Mededelingen 34: 17-22.

BAILlET Y. \& GUICHERD G. 2018. - Dossier de présentation de la Liste rouge Rhopalocères \& Zygènes de Rhône-Alpes. Flavia APE, Trept, $19 \mathrm{p}$.

Baillet Y., Marciau R. \& Grossi J.-L. 2015. — Plan isérois d'action en faveur du genre Maculinea 2015-2020 (déclinaison du plan national d'actions Maculinea). Flavia APE, Conservatoire d'espaces naturels Isère - Avenir, Trept, 99 p.
Barataud J. 2019. - Les Orthoptères en Limousin. Bilan des connaissances et perspectives. Société Entomologique du Limousin, Rencontres faune-limousin du 16 novembre 2019, Chanteix, 18 p.

BARAUD J. 1992. - Coléoptères Scarabaeoidea d'Europe. Fédération française des Sociétés de Sciences naturelles (coll. Faune de France; 78), Paris; Société linnéenne, Lyon, 856 p.

Barrier Y., Baudin B., Duval O., Landemaine D., Tréguier J. \& Mayenne Nature Environnement 2015. - Papillons de la Mayenne - Guide atlas desRhopalocères. Mayenne Nature Environnement, Louverné, 256 p.

Baur B., Baur H., Roesti C., Roesti D. \& Thorens P. 2006. Sauterelles, Grillons et Criquets de Suisse. Éditions Haupt, Berne, $352 \mathrm{p}$.

BAZELET C. S. 2011. - Grasshopper Bioindicators of Effective Large Ecological Networks. PhD Dissertation (Entomology), University of Stellenbosch, $236 \mathrm{p}$.

BAZNAT 2020. — Base naturaliste en Midi-Pyrénées. http:// www.baznat.net/pub/obs_all.php?g=a7, dernière consultation septembre 2020.

Bellmann H. \& LuQueT G.-C. 1995. - Guide des sauterelles, grillons et criquets d'Europe occidentale. Delachaux \& Niestlé, Paris, $383 \mathrm{p}$.

BENCE S. (coord.) 2014. - Liste Rouge régionale des Rhopalocères et Zygènes de Provence-Alpes-Côte d'Azur. Conservatoire d'espaces naturels Provence-Alpes-Côte d'Azur, Sisteron, $22 \mathrm{p}$.

BENCE S. (coord.) 2018. - Liste rouge des Orthoptères de ProvenceAlpes-Côte d'Azur. Réalisée selon la méthodologie et la démarche de l'UICN. Conservatoire d'Espaces Naturels de Provence-AlpesCôte d'Azur, Sisteron, 34 p. + Annexes.

Bence S. 2021. - Premier bilan des connaissances du Criquet hérisson Prionotropis azami. Conservatoire d'espaces naturels ProvenceAlpes-Côte d'Azur, Sisteron, 26 p.

BenCE S. \& Richaud S. (coord.) 2020. - Atlas des papillons de jour \& Zygènes de Provence-Alpes-Côte d'Azur. Le Naturographe, Gap, $544 \mathrm{p}$.

Bense U. 1995. - Longhorn Beetles: Illustrated Key to the Cerambycidae and Vesperidae of Europe. Margraf Verlag, Weikersheim, 512 p.

Bensettiti F. \& Puissauve R. 2015. - Résultats de l'évaluation de l'état de conservation des habitats et des espèces dans le cadre de la directive Habitats-Faune-Flore en France. Rapportage "article 17". Période 2007-2012. MNHN-SPN, MEDDE, Paris, 204 p.

Berger P. 2012. - Coléoptères Cerambycidae de la faune de France continentale et de Corse. Actualisation de l'ouvrage d'André Villiers, 1978. Association Roussillonnaise d'Entomologie, Perpignan, 664 p.

Bergerot B. 2010. - Fonctionnement des communautés de Rhopalocères en milieux urbain et périurbain. Thèse de doctorat de l'Université Pierre et Marie Curie, spécialité Écologie (École doctorale Diversité du Vivant), Paris, 209 p.

BÉTARD F. 2016. - Le Criquet des Ajoncs, Gomphocerippus binotatus ssp. armoricanus, en Vendée et Deux-Sèvres. Données nouvelles sur la répartition et l'écologie d'un Orthoptère menacé. Le Naturaliste Vendéen 12: 87-95.

BijiaOui R. 1986. - Atlas des Longicornes de France. La Duraulié, Réalmont, $56 \mathrm{p}$.

BIODIV' PAYS DE LA LOIRE 2020. - Espèces observées pour le taxon Orthoptera (Ordre). http://www.biodiv-paysdelaloire.fr/ liste/185375, dernière consultation septembre 2020.

Biоторе 2018. - Volet naturel de l'étude d'impact du projet de création d'aménagement sur les sous-bassins versants Garonne et Peyron (Var): bassin d'orage de Vaulongue. Cavem (Rapport ; no contrat 2017 631), Le Cannet des Maures, 194 p.

Bonato L., IORIO E. \& Minelli A. 2011. - The centipede genus Clinopodes C. L. Koch, 1847 (Chilopoda, Geophilomorpha, Geophilidae): reassessment of species diversity and distribution, with a new species from the Maritime Alps (France). Zoosystema 33 (2): 175-205. https://doi.org/10.5252/z2011n2a3

Bonato L., Chagas Junior A., EDGECOMBe G. D., LeWIS J. G. E., Minelli A., Pereira L. A., Shelley R. M., Stoev P. \& Zap- 
PAROli M. 2016. - ChiloBase 2.0 - A World Catalogue of Centipedes (Chilopoda). https://chilobase.biologia.unipd.it, dernière consultation le 10 décembre 2020.

Bordat P., Bouyon H., Dolhem F., Huchet J. B., Jiroux E., Keith D., Moncoutier B. \& Prévost P. 2020. - Faune des Coléoptères de Corse, 2, Lucanidae, Trogidae, Geotrupidae, Hybosoridae, Chironidae, Aphodiidae, Scarabaeidae. Éditions Magellanes, Conflans-Sainte-Honorine, 206 p.

Borges P. A. V., LAMElas-Lopez L., AMORim I. R., DanielCZaK A., Boieiro M., Rego C., Wallon S., Nunes R., Cardoso P. \& HoCHKIRCH A. 2019. - Species conservation profiles of cave-dwelling arthropods from Azores, Portugal. Biodiversity Data Journal 7: 1-89. https://doi.org/10.3897/BDJ.7.e32530

Borisov S. N. 2012. - Migrant Dragonflies in Middle Asia. 3. Pantala flavescens (Fabricius, 1798) (Odonata, Libellulidae). Euroasian Entomological Journal 11 (1): 37-41.

Bouchard P., Bousquet Y., Davies A. E., Alonso-Zarazaga M. A., Lawrence J. F., Lyal C. H. C., Newton A. F., ReID C. A. M., SCHMitT M., ŚLIPIŃSKi S. A. \& SMITH A. B. T. 2011. - Family-group names in Coleoptera (Insecta). ZooKeys 88: 1-972. https://doi.org/10.3897/zookeys.88.807

Boudot J.-P. \& Kalkman V. J. 2015. - Atlas of the European Dragonflies and Damselflies. KNNV publ., Zeist, $381 \mathrm{p}$

Bousquet Y., Thomas D. B., Bouchard P., Smith A. D., Aalbu R. L., Andrew Johnston M. \& Steiner W. E. JR. 2018. - Catalogue of Tenebrionidae (Coleoptera) of North America. Zookeys 728: 1-455. https://doi.org/10.3897/zookeys.728.20602

BOUYON H. 2014. — Famille Tenebrionidae Latreille, 1802, sousfamille Alleculinae Laporte de Castelnau, 1840, in TRONQUET M. (éd.), Catalogue des Coléoptères de France. Association Roussillonnaise d'Entomologie, Perpignan: 535-537.

Bretagne Vivante 2018. - Atlas de répartition provisoire des Orthoptères, Phasmes, Mantes et Forficules de Bretagne. Bretagne Vivante, Brest, $20 \mathrm{p}$.

BROWN K. S. 1997. - Diversity, disturbance, and sustainable use of Neotropical forests: insects as indicators for conservation monitoring. Journal of Insect Conservation 1: 25-42. https://doi. org/10.1023/A:1018422807610

Brown A. C. \& OdendaAl F. J. 1994. - The biology of Oniscid isopoda of the genus Tylos. Advances in Marine Biology 30: 89-153. https://doi.org/10.1016/S0065-2881(08)60062-0

BRUSTEL H. 2002. - Coléoptères saproxyliques et valeur biologique des forêts françaises. Perspectives pour la conservation du patrimoine naturel. Thèse de Doctorat de l'Institut National Polytechnique de Toulouse (Spécialité: Sciences Agronomiques), 327 p.

Brustel H., Berger P. \& COCQuempot C. 2002. - Catalogue des Vesperidae et des Cerambycidae de la faune de France (Coleoptera). Annales de la Société entomologique de France (N. S.) 38 443-461. https://doi.org/10.1080/00379271.2002.10697354

Brustel H., Braud Y., Gouix N., Gazay C., Noblecourt T., Valladares L., Vignon V. \& Touroult J. 2019. — Proposition de protocoles pour la surveillance de l'état de conservation de sept Coléoptères saproxyliques de la Directive Habitats-Faune-Flore. Naturae 2019 (7): 175-210. https://doi.org/10.5852/naturae2019a7

Buord M., David J., Garrin M. \& IliOu B. 2017. - Atlas des papillons diurnes de Bretagne. Locus Solus, Châteaulin, 324 p.

Buse J., SCHRÖDER B. \& ASSMANN T. 2007. - Modelling habitat and spatial distribution of an endangered longhorn beetle - A case study for saproxylic insect conservation. Biological Conservation 137 (3): 372-381. https://doi.org/10.1016/j.biocon.2007.02.025

Cálix M., AleXander K. N. A., Nieto A., Dodelin B., Soldati F., Telnov D., VazQuez-Albalate X., Aleksandrowicz O., Audisio P., Istrate P., JansSon N., Legakis A., LiberTo A., Makris C., Merkl O., Mugerwa Pettersson R., SChlaghamerSky J., Bologna M. A., Brustel H., Buse J., Novák V. \& PURCHART L. 2018. - European Red List of Saproxylic Beetles. UICN, Brussels, $19 \mathrm{p}$.
Cardoso P., Erwin T. L., Borges P. A. V. \& New T. R. 2011. The seven impediments in invertebrate conservation and how to overcome them. Biological Conservation 144 (11): 2647-2655. https://doi.org/10.1016/j.biocon.2011.07.024

Cardoso P., Borges P. A. V., Triantis K. A. \& Ferrández M.-A. 2012. - The underrepresentation and misrepresentation of invertebrates in the IUCN Red List. Biological Conservation 149: 147-148. https://doi.org/10.1016/j.biocon.2012.02.011

Cardoso P., Barton P. S., Birkhofer K., Chichorro F., Deacon C., Fartmann T., Fukushima C. S., Gaigher R., Habel J. C., Hallmann C. A., Hill M. J., Hochkirch A., Kwak M. L., Mammola S., Noriega J. A., Orfinger A. B., Pedraza F., Pryke J. S., Roque F. O., Settele J., Simaika J. P., Stork N. E., Suhling F., Vorster C. \& Samways M. J. 2020. - Scientists' warning to humanity on insect extinctions. Biological Conservation 242: 1-12. https://doi.org/10.1016/j. biocon.2020.108426

Carpaneto G. M., Mazziotta A. \& Valerio L. 2007. — Inferring species decline from collection records: roller dung beetles in Italy (Coleoptera, Scarabaeidae). Diversity and Distributions 13: 903-919. https://doi.org/10.1111/j.1472-4642.2007.00397.x

Chauvin H. 2020. - Le nettoyage mécanique des plages dévaste leur biodiversité. Reporterre, le quotidien de l'Écologie, 6 juin 2020. https://reporterre.net/Le-nettoyage-mecanique-desplages-devaste-leur-biodiversite, dernière consultation le 10 janvier 2021.

Chevassus-Au-Louis B. 2005. - Les enjeux de la biodiversité animale. Bulletin de l'Académie vétérinaire de France 158: 91-110.

Cigliano M. M., Braun H., Eades D. C. \& OtTe D. 2021. Orthoptera Species File. Version 5.0/5.0. http://orthoptera.speciesfile.org, dernière consultation le 10 janvier 2021.

Cocouempot C., Desbles F., Mouttet R. \& Valladares L. 2019. - Xylotrechus chinensis (Chevrolat, 1852), nouvelle espèce invasive pour la France métropolitaine (Coleoptera, Cerambycidae, Clytini). Bulletin de la Société entomologique de France 124 (1): 27-32. https://doi.org/10.32475/bsef_2064

ColAs G. 1974. - Guide de l'entomologiste - L'entomologiste sur le terrain, préparation, conservation des Insectes et des collections. Éditions N. Boubée \& Cie, Paris, 328 p.

Coray A. \& Thorens P. 2001. - Orthoptères de Suisse: clé de détermination. Centre suisse de cartographie de la faune (coll. Fauna Helvetica 5), Neuchâtel, 236 p.

CORBET P. S. 1993. - Are Odonata useful as bioindicators? Libellula 12: 91-102.

COTREL N. (coord.) 2019. - Liste rouge régionale des Rhopalocères du Poitou-Charentes. Poitou-Charentes Nature, UICN, Fontainele-Comte, $15 \mathrm{p}$.

Coulmin A., Lévêque A., Bensettiti F. \& Gazay C. 2020. Biodiversité rare ou menacée: peu d'améliorations depuis 2007. DataLab, Commissariat général au développement durable, Paris, 4 p. Couturier T., Mourguiart B., Mansons J., Braud Y., CombrisSON D., JAILlOuX A. \& BESNARD A. 2020. - Suivi des déplacements altitudinaux des communautés d'Orthoptères en lien avec le changement climatique dans les parcs nationaux du Mercantour et des Écrins. Rapport méthodologique, protocole version 1 . Coopération OFB-CEFE, Montpellier, 44 p.

D'Amico F., Darblade S., Avignon S., Blanc-Manel S. \& ORMEROD S. J. 2004. - Odonates as indicators of Shallow lake restoration by liming: comparing adult and larval responses. Restoration Ecology 12 (3): 439-446. https://doi.org/10.1111/j.10612971.2004.00319.x

DAEDEN J. 2015. - Analyse des pressions anthropiques sur l'environnement littoral européen et français. Thèse de doctorat en Géographie et Science de l'environnement, Université de la Rochelle, 294 p. https://tel.archives-ouvertes.fr/tel-01373455

DALENS H. 1998. — Endémisme pyrénéen : sur une nouvelle espèce épigée du genre Oritoniscus: O. rousseti n. sp. (Crustacea, Isopoda, Oniscidea). Revue suisse de zoologie 105 (2): 339-343. 
Dalens H., Rousset A. \& Fournier D. 1996. — Les formes épigées du genre Oritoniscus (Crustacea, Isopoda, Oniscidea). I. Le complexe Oritoniscus flavus. Revue suisse de zoologie 103 (3): 623-641.

Dalens H., Rousset A. \& Fournier D. 1997. - Les espèces épigées du genre Oritoniscus (Crustacea, Isopoda, Oniscidea). II. Le complexe Oritoniscus bonadonai-pyrenaeus-remyi. Revue suisse de zoologie 104 (4): 727-749.

DaligauX J. 2003. - Urbanisation et environnement sur les littoraux: une analyse spatiale. Rives méditerranéennes 15: 1-7.

DAVID J.-F. 2014. - The role of litter-feeding macroarthropods in decomposition processes: a reappraisal of common views. Soil Biology and Biochemistry 76: 109-118. https://doi.org/10.1016/j. soilbio.2014.05.009

De la Huz J. E. R., Duarte C. \& Contreras H. 2006. - Algal wrack deposits and macroinfaunal arthropods on sandy beaches of the Chilean coast. Revista Chilena de Historia Natural 79: 337-351. https://doi.org/10.4067/S0716-078X2006000300006

DeFaut B. 1999 a. - La détermination des Orthoptères de France. B. Defaut, Bédeilhac, 83 p.

Defaut B. 1999b. - Synopsis des Orthoptères de France. Deuxième édition, révisée et augmentée. Matériaux entomocénotiques hors-série: 187.

Defaut B. \& Morichon D. 2015. - Criquets de France (Orthoptera, Caelifera). Vol. 1 : généralités, systématique (première partie): Caelifera autres que Gomphocerinae (fascicule a et b). Fédération française des sociétés de sciences naturelles, Paris, 695 p.

Dehondt F. (coord.) 2019. - Connaissance des Orthoptères de Franche-Comté: bilan et perspectives (hiver 2018-2019). Office pour les Insectes et leur environnement de Franche-Comté, Conservatoire botanique national de Franche-Comté - Observatoire régional des Invertébrés, Besançon, 14 p. + Annexes.

Deliry C. \& Faton J.-M. 2017. — Ascalaphes de France. Histoires naturelles 10: 1-15.

Delorme Q. 2013. — Éléments d'écologie et de répartition d'une espèce endémique peu connue: Amedegnatiana vicheti (Delmas \& Rambier, 1950) (Orthoptera: Tettigoniidae, Tettigoniinae). Matériaux orthoptériques et entomocénotiques 18: 59-65.

Derjanschi V. \& Péricart J. 2005. - Hémiptères Pentatomoidea euro-méditerranéens. Vol. 1: Généralités, systématique: première partie. Fédération française des sociétés de sciences naturelles, Paris, $494 \mathrm{p}$.

DEWULF L. \& HOUARD X. (coord.) 2016. — Liste ronge régionale des Rhopalocìres et des Zygènes d'Île-de-France. Natureparif, Office pour les Insectes et leur environnement, Association des Lépidoptéristes de France, Paris, 88 p.

Dias N., Sprung M. \& Hassall M. 2005. - The abundance and life histories of terrestrial isopods in a salt marsh of the Ria Formosa lagoon system, southern Portugal. Marine Biology 147: 1343-1352. https://doi.org/10.1007/s00227-005-0033-2

DijKstra K. \& LEWIngton R. 2007. — Guide des Libellules de France et d'Europe. Delachaux et Niestlé, Paris, 320 p.

DOUCET G. 2016. - Clé de détermination des exuvies des Odonates de France. $3^{e}$ édition. Société française d'Odonatologie, Boisd'Arcy, $68 \mathrm{p}$.

DREAL Nouvelle-Aquitaine \& SociÉTÉ ENTOMOlogiQue DU LimOUSIN 2014. - Grand capricorne Cerambyx cerdo (Linnaeus, 1758). Service Valorisation, Évaluation des Ressources et du Patrimoine Naturel (VERPN/DREAL), Limoges, $2 \mathrm{p}$.

DREAL PACA 2013. - Atlas du littoral de Provence-Alpes-Côte d'Azur. CETE Méditerranée \& CETE Normandie-Centre, Aixen-Provence \& le Grand-Quevilly, 269 p.

DuboIs G. 2009. - Écologie des Coléoptères saproxyliques: biologie des populations et conservation d'Osmoderma eremita (Coleoptera: Cetoniidae). Thèse de doctorat de l'Université de Rennes 1, 216 p. + Annexes.

DumonT H. J. \& DumONT S. 1969. - A biometrical analysis of the dragonfly Ischnura elegans (Vander Linden) with special reference to its chloride-tolerance and generation number. Biologisch jaarboek Dodonaea 37: 50-60.
Dunger W. \& Voigtländer K. 2009. - Soil fauna (Lumbricidae, Collembola, Diplopoda and Chilopoda) as indicators of soil eco-subsystem development in post-mining sites of eastern Germany - A review. Soil Organisms 81 (1): 1-51.

DuPONT P. 2010a. - Plan national d'actions en faveur des Maculinea. Office pour les Insectes et leur environnement. Ministère de l'Écologie, du Développement durable, des Transports et du Logement, Paris, 138 p.

DUPONT P. 2010b. - Plan national d'actions en faveur des Odonates. Office pour les Insectes et leur environnement, Société Française d'Odonatologie, Ministère de l'Écologie, de l'Énergie, du Développement durable et de la Mer, Paris, $170 \mathrm{p}$.

DusOUlier F. 2006. — La compréhension des dynamiques spatiotemporelles chez les Orthoptères: la biohistoire au secours des naturalistes. Symbioses nouvelle série 17: 1721.

Dusoulier F. \& Lupoli R. 2006. — Synopsis des Pentatomoidea Leach, 1815 de France métropolitaine (Hemiptera, Heteroptera). Nouvelle revue d'entomologie n. s. 23 (1): 1144.

Dusoulier F., Frapa P., Miquet A., Mazuy M., Ducamp P., Damoiseau S., Cherpitel T., Moulet P. \& Elder J.-F. 2020. - Zicrona, association française des hétéroptéristes. zicrona. fr, dernière consultation le 28 décembre 2020.

Dusoulier F., Cochard P.-O., Ducamp P., Bartolucci J.-C., Dhellemmes T., Guimier H., Maurel J.-P., Sannier D. \& Gillbanks C. (sous presse) - Redécouverte de Phimodera humeralis (Dalman, 1823) dans le département des Landes et nouveaux éléments bio-écologiques (Hemiptera, Heteroptera: Scutelleridae). L'Entomologiste.

ESSER J. 2011. - Antheminia lunulata (Goeze, 1778) weiterhin in Brandenburg (Heteroptera, Pentatomidae). Märkischen Entomologischen Nachrichten 13 (1): 111-113.

Faille A., Bourdeau C. \& DeHARVEng L. 2014. - Weak impact of tourism activities on biodiversity in a subterranean hotspot of endemism and its implications for the conservation of cave fauna. Insect Conservation and Diversity 8: 205-215. https://doi. org/10.1111/icad.12097

FAUNE AQUITAINE 2020. - Portail collaboratif sur la biodiversité régionale. https://www.faune-aquitaine.org/index.php?m_id=23, dernière consultation octobre 2020.

Faune Alsace 2020. - Portail des données naturalistes d'Alsace. https://www.faune-alsace.org/, dernière consultation octobre 2020.

Faune Auvergne 2020. - Portail de science participative naturaliste du Réseau Faune Auvergne. https://www.faune-auvergne. org/index.php?m_id=23, dernière consultation octobre 2020.

FAUNe Champagne-ARDENNES 2020. - Portail interactif consacré à la collecte et à la diffusion d'observations naturalistes en Champagne-Ardenne. https://www.faune-champagne-ardenne.org/index. php?m_id=23, dernière consultation octobre 2020.

FAUNE CHER 2020. - Site collaboratif de la LPO Cher. https:// www.faune-cher.org/index.php?m_id=23, dernière consultation octobre 2020.

Faune Languedoc-Roussillon 2020. — Portail d'échange d'informations naturalistes en Languedoc-Roussillon. https://www.faune-lr. org/index.php?m_id=23, dernière consultation octobre 2020.

FAUNE LORRAINE 2020. - Portail des données naturalistes de Lorraine. https://www.faune-lorraine.org/index.php?m_id=23, dernière consultation octobre 2020.

FaUne Touraine 2020. - Site collaboratif de la LPO Touraine. https://www.faune-touraine.org/index.php?m_id=23, dernière consultation octobre 2020.

FLAVIA APE 2018. - Liste rouge régionale. Papillons diurnes de Rhône-Alpes (Rhopalocères et Zygènes). Région Auvergne-RhôneAlpes \& UICN, Lyon, 21 p.

Fontaine B., Bouchet P., VAn Achterberg K., Alonso-Zarazaga M. A., Araujo R., Asche M., Aspöck U., Audisio P., Aukema B., Bailly N., Balsamo M., Bank R. A., Barnard P., Belfiore C., Bogdanowicz W., Bongers T., Boxshall G., Burckhardt D., Camicas J.-L., Chylarecki P., Crucitti P., 
Deharveng L., Dubois A., Enghoff H., Faubel A., Fochetti R., Gargominy O., Gibson D., Gibson R., Gómez López M. S., Goujet D., Harvey M. S., Heller K.-G., Van Helsdingen P., Hoch H., De Jong H., De Jong Y., Karsholt O., Los W., LundqVist L., MagowsKi W., ManCONI R., MaRtens J., Massard J. A., Massard-Geimer G., Mcinnes S. J., Mendes L. F., Mey E., Michelsen V., Minelli A., Nielsen C., Nieto Nafría J. M., Van Nieukerken E. J., Noyes J., Pape T., Pohl H., De Prins W., Ramos M., Ricci C., Roselaar C., Rota E., Schmidt-Rhaesa A., Segers H., Zur Strassen R., SZEPTYCKI A., Thibaud J.-M., ThOMas A., Timm T., VAN Tol J., VERVOORT W. \& WillmanN R. 2007. - The European Union's 2010 target: putting rare species in focus. Biological Conservation 139: 167-185. https://doi.org/10.1016/j.biocon.2007.06.012

France 3 Provence-AlPes-Côte D'Azur 2019. - Les opposants à la carrière de Mazaugues restent mobilisés. Publié le 12 avril 2019. https://france3-regions.francetvinfo.fr/provence-alpescote-d-azur/var/brignoles/opposants-carriere-mazaugues-restentmobilises-1654532.html, dernière consultation le 5 janvier 2021.

FODDAI D. \& MiNELLI A. 1999. - A troglomorphic geophilomorph centipede from southern France (Chilopoda, Geophilomorpha, Geophilidae). Journal of Natural History 33: 267-287. https:// doi.org/10.1080/002229399300416

Gargominy O., Tercerie S., Régnier C., Ramage T., Dupont P., DASZKIEWICZ P. \& PONCET L. 2020a. - TAXREF v14, référentiel taxonomique pour la France: méthodologie, mise en oeuvre et diffusion. UMS PatriNat (OFB-CNRS-MNHN), Paris, $63 \mathrm{p}$.

Gargominy O., Tercerie S., Régnier C., Dupont P., DaszKiewicz P., Léotard G., Antonetti P., Ramage T., Vandel É., PetitTeVille M., Leblond S., IdCZaK L., Boullet V., Denys G., Massary (DE) J.-C., Lévêque A., Jourdan H., Rome Q., Dusoulier F., Touroult J., Savouré-Soubelet A., Barbut J., Canard A., Simian G., Le Divelec R., Haffner P., Meyer C., Van Es J., Poncet R., Demerges D., Mehran B., Horellou A., Moulin N., Ah-Peng C., Bernard J.-F., CaeSar M., Comolet-Tirman J., Courtecuisse R., Delfosse E., Dewynter M., Hugonnot V., Kondratyeva A., Lavocat Bernard E., Lebouvier M., Lebreton E., Malécot V., Moreau P. A., Muller S., Noblecourt T., Pellens R., RobBert Gradstein S., Rodrigues C., Rouhan G. \& Véron S. 2020b. - TAXREF v14.0, référentiel taxonomique pour la France. UMS PatriNat (OFB-CNRS-MNHN), Paris. https://inpn.mnhn. $\mathrm{fr} /$ telechargement/referentielEspece/taxref/14.0/menu, dernière consultation le 30 janvier 2021.

Gayet G., Baptist F., Maciejewski L., POnCet R. \& Bensettiti F. 2018. - Guide de détermination des habitats terrestres et marins de la typologie EUNIS - Version 1.0. Agence française pour la biodiversité (AFB), Paris, 184 p. + Annexes.

GeOFFrOY J.-J. \& IORIO É. 2019. - Les Myriapodes, in JOHANET A. \& KaBOUCHE B. (éds), La faune des Bouches-du-Rhône. Biotope éditions, Mèze: 325-329.

GOUVERnEUR X. \& GUÉRARD P. 2011. — Les Longicornes armoricains - Atlas des Coléoptères Cerambycidae des départements du Massif Armoricain. Invertébrés armoricains 7: 1-224.

Grand D., Boudot J.-P. \& Doucet G. 2019. - Cahier d'identification des Libellules de France, Belgique, Luxembourg et Suisse - 2e édition. Biotope, Mèze, $152 \mathrm{p}$.

GrGIČ T. \& KOS I. 2005. - Centipede diversity in differently structured forests of Slovenia. Peckiana 4: 49-56.

Gubbay S., Sanders N., Haynes T., Janssen J. A. M., Rodwell J. R., Nieto A., García Criado M., Beal S., Borg J., Kennedy M., Micu D., Otero M., Saunders G. \& Calix M. 2016. - European Red List of Habitats. Part 1 Marine Habitats. Publications Office of the European Union, Luxembourg, 46 p. https://doi.org/10.2779/032638

Guilloton J.-A. 2018. — Biohistoire des papillons. Diversité et conservation des Lépidoptères Rhopalocères de la Loire-Atlantique et de la Vendée. $2^{e}$ état des lieux. Atlas entomologique régional, Nantes, $119 \mathrm{p}$.
Günther H., Hoffmann H.-J., Melber A., Remane R., Simon H. \& WinKELMANN H., 1998. - Rote Liste der Wanzen (Heteroptera). Schriftenreihe für Landschaftspflege und Naturschutz 55: 235-251.

HANSKI I. \& CAMBEForT Y. (éds.) 1991. — Dung Beetle Ecology. Princeton University Press, Princeton, 481 p.

HinNeKINT B. O. N. 1987. - Population dynamics of Ischnura e. elegans (Vander Linden) (Insecta: Odonata) with special reference to morphological colour changes, female polymorphism, multiannual cycles and their influence on behaviour. Hydrobiologia 146 (1): 3-31. https://doi.org/10.1007/BF00007574

Hochkirch A., Nieto A., García Criado M., Cálix M., Braud Y., Buzzetti F. M., Chobanov D., Odé B., Presa Asensio J. J., Willemse L., Zuna-Kratky T., Barranco Vega P., Bushell M., Clemente M. E., Correas J. R., Dusoulier F., Ferreira S., Fontana P., García M. D., Heller K.-G., Iorgu I. S., Ivković S., Kati V., Kleukers R., KrišTín A., LEMONNIER-DarCemont M., Lemos P., Massa B., Monnerat C., Papapavlou K. P., Prunier F., Pushkar T., Roesti C., Rutschmann F., Şirin D., Skejo J., Szövényi G., Tzirkalli E., Vedenina V., Barat Domenech J., Barros F., Cordero Tapia P. J., Defaut B., Fartmann T., Gomboc S., GutiérReZ-Rodríguez J., HoluŠA J., Illich I., Karjalainen S., KoČÁREK P., KorsunOVSKaya O., Liana A., López H., Morin D., Olmo-Vidal J. M., Puskás G., SavitSKY V., STAlling T. \& TumbrincK J. 2016. - European Red List of Grasshoppers, Crickets and Bush-crickets. Publications Office of the European Union, Luxembourg, 57 p. + Annexes.

HoK YAU TANG D. \& VISCONTI P. 2020. — Biases of Odonata in Habitats Directive: trends, trend drivers, and conservation status of European threatened Odonata. Insect Conservation and Diversity 14 (1): 1-14. https://doi.org/10.1111/icad.12450

HouARD X. (coord.) 2020. - Plan national d'actions en faveur des "libellules»-Agir pour la préservation des odonates menacés et de leurs habitats 2020-2030. Office pour les insectes et leur environnement, DREAL Hauts-de-France, Ministère de la transition écologique, Lille, $66 \mathrm{p}$.

HOUARD X. \& JAULIN S. (coord.) 2018. — Plan national d'actions en faveur des "Papillons de jour". Agir pour la préservation de nos Lépidoptères diurnes patrimoniaux 2018-2028. Office pour les Insectes et leur environnement, DREAL Auvergne-Rhône-Alpes, Ministère de la Transition écologique et solidaire, Lyon, 64 p.

IMAGO 2017. - Atlas préliminaire des Orthoptères d'Alsace. Faune Alsace document 1a: 1-87.

INVENTAIRE NATIONAL DU PATRIMOINE NATUREL 2020. — Atlas des rhopalocères $d u$ Nord et du Pas-de-Calais. https://inpn.mnhn.fr/ espece/jeudonnees/3492, dernière consultation octobre 2020.

InVENTAIRE NATIONAL DU PATRIMOINE NATUREL/OPEN OBS 2020. Portail français d'accès aux données d'observation sur les espèces. https://openobs.mnhn.fr, dernière consultation décembre 2020

IORIO É. 2010a. - Description d'une nouvelle sous-espèce de Cryptops umbricus Verhoeff, 1931 (Chilopoda, Scolopendromorpha, Cryptopidae). Bulletin de la Société linnéenne de Bordeaux 144 37 (4): 471-481.

IORIO É. 2010b. - Les Lithobies et genres voisins de France (Chilopoda, Lithobiomorpha). Révision de plusieurs espèces méconnues et nombreux apports inédits à la connaissance du genre Lithobius Leach, 1814. Avec une clé des familles, des genres et de toutes les espèces de l'ordre. Revue de l'Association Roussillonnaise d'Entomologie 19: 1-104.

IORIO É. 2014. - Catalogue biogéographique et taxonomique des Chilopodes (Chilopoda) de France métropolitaine. Mémoires de la Société linnéenne de Bordeaux 15: 1-372.

IORIO É. \& GEOFFrOY J.-J. 2008. — Les scolopendromorphes de France (Chilopoda, Scolopendromorpha) : identification et distribution géographique des espèces. Riviéra scientifique 91: 73-90.

Iorio É. \& GeOfFroY J.-J. 2019. —Étude des Chilopodes de Païolive (Ardèche, France) et description d'une nouvelle espèce du genre Lithobius Leach, 1814 (Myriapoda, Chilopoda). Bulletin 
de la Société entomologique de France 124 (2): 109-126. https:// doi.org/10.32475/bsef_2081

Iorio É. \& Labroche A. 2015. — Les Chilopodes (Chilopoda) de la moitié nord de la France: toutes les bases pour débuter l'étude de ce groupe et identifier facilement les espèces. Invertébrés armoricains 13: 1-108.

IORIO É. \& PÉTILLON J. 2020. — Influence of small- and large-scale ecological factors on the centipedes (Chilopoda) assemblages of Armorican forests (NW France). Vie et Milieu 70 (1): 79-87.

IORIO É., DECOIN R. \& Claude J. 2019. — Une espèce nouvelle pour la France découverte dans la Réserve naturelle nationale du Lac du Remoray (Doubs) : Eupolybothrus tridentinus (Fanzago, 1874) (Chilopoda, Lithobiomorpha, Lithobiidae). Bulletin de la Société linnéenne de Bordeaux 154 (n. s.) 47 (3/4): 203-209.

Iorio É., Geoffroy D. \& Pétillon J. 2020. - Distribution and indicator value of intertidal centipedes from Mediterranean beaches within and around Port-Cros National Park (Southern France), with proposal of a simplified monitoring (Chilopoda). Bulletin de la Société entomologique de France 125 (1): 41-62. https://doi.org/10.32475/bsef_2107

Iorio É., Zapparoli M., Ponel P. \& Geoffroy J.-J. 2015. Les Myriapodes Chilopodes (Chilopoda) du Parc national du Mercantour, du département des Alpes-Maritimes et de leurs environs: description d'une nouvelle espèce du genre Lithobius Leach, 1814 s.s., synthèse des connaissances et espèces menacées. Zoosystema 37 (1): 211-238. https://doi.org/10.5252/ z2015n1a11

JABIN M. 2008. - Influence of Environmental Factors on the Distribution Pattern of Centipedes (Chilopoda) and other Soil Arthropods in Temperate Deciduous Forests. Göttingen, Cuvillier, 128 p.

JACQUOT P. 2012. - Déesse précieuse (Nehalennia speciosa). Union européenne, Direction régionale de l'Environnement, de l'Aménagement et du Logement de Franche-Comté, Conseil régional de Franche-Comté, Besançon, 2 p.

Janssen J. A. M., Rodwell J. S., García CRIAdo M., Gubbay S., Haynes T., Nieto A., Sanders N., Landucci F., Loidi J., Ssymank A., TahVanainen T., Valderrabano M., Acosta A., Aronsson M., Arts G., Attorre F., Bergmeier E., Bijlsma R.-J., Bioret F., Biţă-Nicolae C., Biurrun I., Calix M., Capelo J., ČArni A., Chytrý M., Dengler J., Dimopoulos P., Essl F., Gardfjell H., Gigante D., Giusso del Galdo G., Hájek M., Jansen F., Jansen J., Kapfer J., MickolajCZAK A., Molina J. A., Molnár Z., Paternoster D., Piernik A., Poulin B., Renaux B., Schaminée J. H. J., Šumberová K., Toivonen H., Tonteri T., TSIRIPIDIS I., TZONEV R. \& VAlachoviČ M. 2016. — European Red List of Habitats. Part 2. Terrestrial and freshwater habitats. European Union Publications office, Luxembourg, 38 p. https:// doi.org/10.2779/091372

JAULIN S. \& SOLDATI F. 2005. — Les dunes littorales du LanguedocRoussillon. Guide méthodologique d'évaluation de leur état de conservation à travers l'étude des cortèges spécialisés de Coléoptères. OPIE-LR, DIREN-LR, Millas, 58 p.

Joly F.-X., COQ S., COUlis M., DAVID J.-F., HÄTtensChWILER S., Mueller C. W., Prater I. \& SubKe J.-A. 2020. — Detritivore conversion of litter into faeces accelerates organic matter turnover. Communications Biology 3: 1-9.

Kalkman V. J., Boudot J.-P., Bernard R., Conze K.-J., De Knijf G., Dyatlova E., Ferreira S., Jović M., Ott J., RisERVATO E. \& SAHLÉN G. 2010. — European Red List of Dragonfiles. Publications Office of the European Union, Luxembourg, 19 p. + Annexes.

Karam-Gemael M., Jungueira Izzo T. \& Chagas-Junior A. 2018. - Why be red listed? Threatened Myriapoda species in Brazil with implications for their conservation. ZooKeys 741: 255-269. https://doi.org/10.3897/zookeys.741.21971

KARPIŃSKI L., SZCZEPAŃSKI W. \& KRUSZELNICKI L. 2020. — Revision of the Ropalopus ungaricus/ insubricus group (Coleoptera: Cerambycidae: Callidiini) from the western Palaearctic region.
Zoological Journal of the Linnean Society 189 (4): 1176-1216. https://doi.org/10.1093/zoolinnean/zlz154

KMENT P. \& VILÍMOVÁ J. 2005. - Heteroptera (ploštice), in FARKaČ J., Král D. \& ŠKorpíK M. (éds), Červený seznam ohrožených druhü České republiky. Bezobratli. Red list of threatened species in the Czech Republic. Invertebrates. Agentura ochrany prírody a krajiny ČR, Praha: 139-146.

LAFRANCHIS T., JUTZELER D., GUILlOSSON J.-Y., KAN P. \& KAN B. 2015. - La vie des papillons. Écologie, biologie et comportement des Rhopalocères de France. Diatheo, Montfaucon, $751 \mathrm{p}$.

LAMBRET P. \& BOUDOT J.-P. 2013. - Hemianax ephippiger (Burmeister, 1839) (Odonata, Anisoptera: Aeshnidae): présentation générale. Martinia Hors-série Hemianax ephippiger - migration 2011: 13-27.

le Berre M., Noble V., Pires M., Médail F. \& Diadema K. 2021. - How to hierarchise species to determine priorities for conservation action? A critical analysis. Biodiversity and Conservation 28: 3051-3071. https://doi.org/10.1007/s10531-019-01820-w

LE DIVELEC R. 2021. - Sur la présence en France de certaines espèces d'Apoïdes (Hymenoptera, Andrenidae, Colletidae, Megachilidae, Psenidae). Bulletin de la Société entomologique de France 126 (1): 103-122. https://doi.org/10.32475/bsef_2176

Le Féon V., Geslin B., Al Hassan D., Aviron S., Dufrêne E., Genoud D., Guilbaud L., Guilloux T., Houédrys L., JéGat R., Mahé G., Paris D., Requier F. \& Vaissière B. 2016. - Connaissances sur les abeilles sauvages (Hymenoptera, Apoidea) dans le Massif armoricain: recensement des publications existantes et restitution des données de distribution apportées par trois programmes de recherche depuis 2000. Invertébrés armoricains 14:31-51.

LEATHER S. R. 2013. - Institutional vertebratism hampers insect conservation generally; not just saproxylic beetle conservation. Animal Conservation 16 (4):379-380. https://doi.org/10.1111/ acv. 12068

Leipelt K. G. \& SuHLing F. 2001. - Habitat selection of larval Gomphus graslinii and Oxygastra curtisii (Odonata: Gomphidae, Corduliidae). International Journal of Odonatology 4: 23-34. https://doi.org/10.1080/13887890.2001.9748155

LERAUT P. 2016. - Papillons de jour d'Europe et des contrées voisines. NAP éditions, Verrières-le-Buisson, $1111 \mathrm{p}$.

Lessieur D. \& Lupoli R. 2018. - Phimodera humeralis (Dalman, 1823) : observation en Gironde d'une punaise rarissime non revue en France depuis plus de 80 ans (Hemiptera Scutelleridae). L'Entomologiste 73 (5): 337-340.

LEWIS J. G. E. 1997. — The possible factors affecting the distribution and abundance of the centipedes Lithobius variegatus Leach and Lithobius forficatus (Linn.) in the British Isles. Bulletin of the British Myriapod Group 13: 14-18.

LORTHIOIS M. (coord.) 2015. — Liste rouge des papillons diurnes \& Zygènes de Haute-Normandie. Indicateurs pour l'Observatoire de la Biodiversité de Haute-Normandie, Rouen, 17 p.

Louboutin B., Jaulin S., Charlot B. \& Danflous S. (coord.), 2019. - Liste rouge des Lépidoptères Rhopalocères et Zygènes d'Occitanie. Rapport d'évaluation. Opie, CEN MP \& CEN LR, Montferrier, Lez, 304 p.

LuMARET J.-P. 1990. - Atlas des Coléoptères Laparosticti de France. Muséum national d'Histoire naturelle, Paris, $419 \mathrm{p}$.

LUMARET J.-P. 2001. — Impact des produits vétérinaires sur les Insectes coprophages: conséquences sur la dégradation des excréments dans les pâturages. Bulletin de la Société française de Parasitologie 19 (1): 1-81.

Lumaret J.-P., Lobo J. M. \& Jay-Robert P. 1996. - Catalogue et Atlas des Scarabeides Laparosticti endémiques de France. Collection Patrimoines Naturels, n²6, MNHN et OPIE, Paris, 70 p.

Lupoli R. \& Dusoulier F. 2015. - Les punaises Pentatomoidea de France. Éditions Ancyrosoma, Fontenay-sous-Bois, 429 p.

Maciejewski L., Seytre L., Van Es J. \& Dupont P. 2015. - Etat de conservation des habitats agropastoraux d'intérêt communautaire, méthode d'évaluation à l'échelle du site. Guide d'application. Version 3. MNHN-SPN, Paris, 194 p. 
Magnien P. \& Ponel P. 2011. — Les espèces du genre Ochetostethus Fieber, 1860 en France. Découverte d'Ochetostethus melonii Rizzotti-Vlach, 2000 en Corse, nouveau pour la faune de France (Heteroptera, Cydnidae, Sehirinae). Nouvelle revue d'entomologie nouvelle série 27 (4): 303-310.

Mammola S., Riccardi N., Prié V., Correia R., Cardoso P., LOPES-Lima M. \& SOUSA R. 2020. — Towards a taxonomically unbiased European Union biodiversity strategy for 2030 . Proceedings of the Royal Society B: Biological Sciences 287: 1-12. https://doi.org/10.1098/rspb.2020.2166

MANGER R. \& Dingemanse N. J. 2007. - Survival and biotope selection of Sympecma paedisca in a winter habitat in the Netherlands. Brachytron 11 (1): 52-62.

ManNe L. L. \& Williams P. H. 2003. - Building indicator groups based on species characteristics can improve conservation planning. Animal Conservation 6: 291-297. https://doi.org/10.1017/ S1367943003003354

Matushrina N. A. \& Gorb S. N. 2002. - A check-list of substrates for endophytic oviposition of some European dragonflies (Insecta: Odonata). The Kharkov Entomological Society Gazette 10: 108-118.

Menta C. \& Remelli S. 2020. - Soil health and Arthropods: from complex system to worthwhile investigation. Insects 11 : 1-21. https://doi.org/10.3390/insects11010054

Micó E., García-López A., Sánchez A., JuÁrez M. \& Galante E. 2015. - What can physical, biotic and chemical features of a tree hollow tell us about their associated diversity? Journal of Insect Conservation 19 (1): 141-153. https://doi.org/10.1007/ s10841-015-9754-9

Monnerat C. \& MaibaCH A. 2014. - Fiches de protection espècesLibellules - Lestes dryas. Groupe de travail pour la conservation des Libellules de Suisse, CSCF, Neuchâtel; Office fédéral de l'environnement, Berne, $5 \mathrm{p}$.

Mulsant É. \& Rey C. 1865. - Histoire naturelle des punaises de France. Scutellérides. F. Savy \& Deyrolle, Paris, 112 p.

Mulsant É. \& REY C. 1866. - Histoire naturelle des punaises de France. II. Pentatomides. F. Savy \& Deyrolle, Paris, 372 p.

NATURAlia 2017. - Projet de reconfiguration de la station d'épuration du Chêne. Commune d'Apt (84). Volet naturel du dossier loi sur l'eau. Rapport nPA170117-SF1, Naturalia, Avignon, 97 p. + Annexes. http://www.vaucluse.gouv.fr/IMG/pdf/3.1_diagnostic_ecologique.pdf, dernière consultation le 30 octobre 2020.

NATURALISTES ANGEVINS 2020. - Inventaire et cartographie des Papillons de jour de Maine-et-Loire (Lépidoptères Rhopalocères et Zygaenidae). http://naturalistesangevins.free.fr/doc_cartographie2020.pdf, dernière consultation octobre 2020.

Nieto A., Roberts S. P. M., Kemp J., Rasmont P., Kuhlmann M., García Criado M., Biesmeijer J. C., Bogusch P., Dathe H. H., De la Rúa P., De Meulemeester T., Dehon M., Dewulf A., Ortiz-Sánchez F. J., Lhomme P., Pauly A., Potts S. G., Praz C., Quaranta M., Radchenko V. G., Scheuchl E., Smit J., Straka J., Terzo M., Tomozi B., Window J. \& MicheZ D. 2014. - European Red List of Bees. Publication Office of the European Union, Luxembourg, 41 p. + Annexes.

Nowicki P., Vrabec V., Binzenhöfer B., Feil J., Zaksek B., Hovestadt T. \& SeTtele J. 2014. - Butterfly dispersal in inhospitable matrix: rare, risky, but long-distance. Landscape Ecology 29: 401-412. https://doi.org/10.1007/s10980-0139971-0

Numa C., Tonelli M., Lobo J. M., Verdú J. R., Lumaret J.-P., Sánchez-Piñero F., Ruiz J. L., Dellacasa M., Ziani S., Arriaga A., Cabrero F., Labidi I., Barrios V., ȘenyüZ Y. \& ANLAŞ S., 2020. - The Conservation Status and Distribution of Mediterranean Dung Beetles. IUCN, Gland, Málaga, 58 p.

Otero M. M., Simeone S., Aljinovic B., Salomidi M., Mossone P., Giunta Fornasin M. E., Gerakaris V., Guala I., Milano P., Heurtefeux H., Issaris Y., Guido M. \& Adamopoulou M. 2018. - POSBEMED: gouvernance et gestion des systèmes plagel dunes à Posidonie. Rapport final, UICN, Malaga, 66 p.
Paulian R. \& Baraud J. 1982. - Faune des Coléoptères de France. II. Lucanoidea et Scarabaeoidea. Lechevalier, Paris, 477 p.

PéRICART J. 2010. - Hémiptères Pentatomoidea euro-Méditerranéens. Vol. 3: systématique: troisième partie: sous-familles Podopinae et Asopinae. Fédération française des sociétés de sciences naturelles, Paris, 291 p.

Perrein C. 2012. - Biohistoire des papillons. Diversité et conservation des Lépidoptères Rhopalocères de la Loire-Atlantique et de la Vendée. Presses universitaires de Rennes, $621 \mathrm{p}$.

PiCARDIE NATURE (coord.) 2016. — Listes rouges régionales de la faune menacée de Picardie. Picardie Nature, Préfet des régions Nord-Pas-de-Calais et Picardie, $41 \mathrm{p}$.

Piedallu C., Perez V., Gégout J. C., Lebourgeois F. \& BerTRAND R. 2009. - Impact potentiel du changement climatique sur la distribution de l'Épicéa, du Sapin, du Hêtre et du Chêne sessile en France. Revue forestière française 61 (6): 567-593.

Platek M., Sebek P., Hauck D. \& CizeK L. 2019. - When is a tree suitable for a veteran tree specialist? Variability in the habitat requirements of the great capricorn beetle (Cerambyx cerdo) (Coleoptera: Cerambycidae). European Journal of Entomology 116: 64-74. https://doi.org/10.14411/eje.2019.007

Poitou-Charentes Nature (coord.) 2009. - Libellules de PoitouCharentes. Poitou-Charentes Nature, Fontenay-le-Compte, $256 \mathrm{p}$

Poitou-Charentes Nature 2019. - Liste rouge du PoitouCharentes: chapitre Orthoptères. Poitou-Charentes Nature, Fontaine-le-Comte, $13 \mathrm{p}$.

Polette P., Abbott C., Gouys J., Jenard P., Juliand P., DarNAUd S. \& Boudot J.-P. 2017. — Premières mentions de Trithemis kirbyi (Odonata: Libellulidae) en France. Martinia 33 (1-2): 15-25.

Ponel P., Marchal M., Geoffroy D. \& Soldati F. 2020. Leptoderis solieri Ferrer, 2015 (= Elenophorus collaris auct. nec Linnaeus, 1767), toujours présent dans le département du Var (Coleoptera, Tenebrionidae). Annales de la Société des Sciences naturelles de Toulon et du Var 72 (2): 53-55.

POSER T. 1990. - The Influence of litter manipulation on the centipedes of a beech wood, in Minelli A. (éd.), Proceedings of the Seventh International Congress on Myriapods. Brill, Leiden: 235-245.

PUISSAUVE R. 2009. - La Diane Zerynthia polyxena Denis \& Schiffermüller, 1775 [Lepidoptera, Papilionidae] et son habitat dans la plaine de Pompignan (France, Gard): définition d'un protocole d'étude standardisé. Rapport de stage de Master 1 en Ingénierie et en gestion de la Biodiversité, Université Montpellier 2, Association Gard Nature, Beaucaire, 24 p. + Annexes.

Purse B. 2002. - The Ecology and Conservation of the Southern Damselfy (Coenagrion mercuriale Charpentier) in Britain. Environment Agency, Bristol, 122 p.

PuTON A. 1881. - Synopsis des Hémiptères-Hétéroptères de France. Deuxième volume. $4 \mathrm{e}$ partie: famille des Pentatomides. Éditions Deyrolle, Paris, 129 p.

Rabitsch W. 2002. — Die Wanzenfauna (Heteroptera) der Sandberge bei Oberweiden im Marchfeld (Niederösterreich). Beiträge zur Entomofaunistik 3: 141-174

RACINE A. \& IORIO É. 2017. - Contribution à la connaissance des Chilopodes du Finistère et des Côtes d'Armor (Myriapoda, Chilopoda). Invertébrés armoricains 16: 3-28.

Racine A. \& Iorio É. (coord.) 2020. — Projet d'atlas des Chilopodes (Chilopoda) des Pays de la Loire: bilan des $5 e$ et 6 e années. GRETIA, Nort-sur-Erdre, 22 p.

RaINETTE 2013. - Étude d'impact, volet faune/flore. Projet d'exploitation de carrière sur la commune de Marly (59). Rapport en sous-traitance de Kaliès pour la Briqueterie Chimot, Valenciennes, 75 p. https:// www.nord.gouv.fr/content/download/41718/286992/file/07\%20 -\%20Etude $\% 20$ faune $\% 20-\% 20$ flore.pdf, dernière consultation le 30 octobre 2020.

RAINETTE 2017. - Renouvellement et extension de carrière alluvionnaire sur les communes de Warluis, Rochy-Condé et Bailleul-sur-Thérain 
(60). Etude d'impact et d'incidences. Volet faunelflore. Rapport d'étude, Rainette, Valenciennes, 229 p. http://www.oise.gouv.fr/ content/download/53415/329051/file/3-Etude\%20ecologique. pdf, dernière consultation le 30 octobre 2020.

RAMSAY A. J. 2013. - Habitat preference and distribution of Rhacognathus punctatus (Hemiptera: Pentatomidae) in Wales. British Journal of Entomology \& Natural History 26: 91-94.

Ribes J. \& Pagola-Carte S. 2013. - Hémiptères Pentatomoidea Euro-Méditerranéens. Vol. 2: Systématique: deuxième partie; sous-famille Pentatominae (suite et fin). Fédération française des sociétés de sciences naturelles, Paris, 423 p.

Rider D. A., SChWERTNER C. F., VilímOVÁ J., Rédei D., KMENT P. \& ThOMAS D. B. 2018. - Higher systematics of the Pentatomoidea, in MC Pherson J. E. (éd), Invasive Stink Bugs and Related Species (Pentatomoidea). Biology, Higher Systematics, Semiochemistry, and Management. CRC Press, Boca Raton/London/New York: 25-201. https://doi.org/10.1201/9781315371221-2

Rintala T. \& Rinne V. 2011. — Suomen Luteet. Tibiale Oy, Helsinki, $352 \mathrm{p}$.

RoBERGE J.-M. \& ANGELSTAM P. 2004. - Usefulness of the umbrella species concept as a conservation tool. Conservation Biology 18 (1): 76-85. https://doi.org/10.1111/J.1523-1739.2004.00450.X

RouQueTte J. R. \& ThOMPSON D. J. 2007. — Patterns of movement and dispersal in an endangered damselfly and the consequences for its management. Journal of Applied Ecology 44 (3): 692-701. https://doi.org/10.1111/j.1365-2664.2007.01284.x

Sainte-Claire Deville J. 1937. - Catalogue raisonné des Coléoptères de France, 3e partie. L'Abeille 36 (3): 265-372.

SAMA G. 2003. - Atlas of the Cerambycidae of Europe and the Mediterranean Area. Vol. 1: Northern, Western, Central and Eastern Europe. British Isles and Continental Europe from France (excl. Corsica) to Scandinavia and Urals. Vít Kabourek, Zlín, 173p.

SANNIER D. 2019. - Une nouvelle espèce de punaise découverte en France dans le département des Pyrénées-Orientales: Eurydema (Eurydema) sea Péricart \& De la Rosa [sic], 2004 (Heteroptera, Pentatomidae). Les cahiers de la fondation Biotope 30: 116.

SARDET E. (coord.) 2018. - Liste rouge des Orthoptères de la région RhôneAlpes. DREAL Auvergne-Rhône-Alpes, Lyon, 32 p. + 4 Annexes.

SARDET É. \& Defaut B. 2004. - Les Orthoptères menacés en France. Liste rouge nationale et Listes rouges par domaines biogéographiques. Matériaux orthoptériques et entomocénotiques 9: $125-137$

SARDET É., ROESTI C. \& BRAUD Y. 2015. — Cahier d'identification des Orthoptères de France, Belgique, Luxembourg \& Suisse. Editions Biotope, Mèze, $304 \mathrm{p}$.

SCHRIDDE P. \& SUHLING F. 1994. — Larval dragonfly communities in different habitats of a Mediterranean running water system. Advances in Odonatology 6: 89-100.

SÉCHET E. \& NoËL F. 2015. - Catalogue commenté des Crustacés Isopodes terrestres de France métropolitaine (Crustacea, Isopoda, Oniscidea). Mémoires de la Société Linnéenne de Bordeaux 16: 1-156.

SFenthourakis S. \& TAiTI S. 2015. - Patterns of taxonomic diversity among terrestrial isopods. ZooKeys 515: 13-25. https:// doi.org/10.3897/zookeys.515.9332

Silva M.-S. \& FERREIRA R. L. 2015. - Cave invertebrates in Espírito Santo state, Brazil: a primary analysis of endemism, threats and conservation priorities. Subterranean Biology 16: 79-102. https:// doi.org/10.3897/subtbiol.16.5227

Simaiakis S. M. \& STRONA G. 2014. — Patterns and processes in the distribution of European centipedes (Chilopoda). Journal of Biogeography 42 (6): 1018-1028. https://doi.org/10.1111/ jbi. 12463

Société D'Histoire naturelle D'AuTun 2015. - Élaboration d'une Liste rouge des Rhopalocères et Zygènes de Bourgogne. Dossier de synthèse, Société d'Histoire naturelle, Autun, 11 p.

SOCIÉTÉ D'HisTOIRE NATURELLE D'AUTUN 2020. — Le groupe orthoptères Bourgogne. https://www.shna-ofab.fr/fr/le-groupe-orthopteresbourgogne_685.html, dernière consultation octobre 2020.
Société entomologique du Limousin 2020. — Actualisation de l'inventaire des papillons de jour du Limousin. http://www. selweb.fr/atlas_rhopalos_etat_des_connaissances.php, dernière consultation octobre 2020 .

SOldATI F. 1995. - Les Tenebrionidae de l'Hérault: inventaire et écologie (Coleoptera). Bulletin de la Société entomologique de France 100 (3): 261-275.

Soldati F. 2007. - Fauna of France and Corsica, Coleoptera Tenebrionidae (Alleculinae excluded). Systematic catalogue and atlas. Mémoires de la Société linnéenne de Bordeaux 6: 1-186.

Soldati F. \& Rougon D. 2017. - Présence de Pimelia muricata Olivier, 1795, en Corse, description d'une nouvelle sous-espèce et découverte d'hybrides naturels avec P. angusticollis Solier, 1836 (Coleoptera, Tenebrionidae, Pimeliinae). Bulletin de la Société entomologique de France 122 (4): 401-408. https://doi. org/10.32475/bsef_1993

SoldATI F. \& Soldati L. 2002. — Catalogue raisonné et illustré des Coléoptères Tenebrionidae des Pyrénées-Orientales (Alleculinae exclus). Revue de l'Association roussillonnaise d'Entomologie 11 (2): 1-44.

SOldATI F. \& SOldATI L. 2014. — Famille Tenebrionidae, in TrONQUET M. (éd.), Catalogue des Coléoptères de France. Association Roussillonnaise d'Entomologie, Perpignan: 535-549.

SoldATI F. \& SoldATI L. (sous presse) - Faune de France métropolitaine, Coléoptères Tenebrionidae (Alleculinae exclus).

SoldATI F., CourTin O., Sudre J. \& SoldATI L. 2019. - Euboeus (Pelorinus) dieckii (Kraatz, 1870), espèce nouvelle pour la faune de France (Coleoptera Tenebrionidae). L'Entomologiste 75 (6): $329-335$.

Soldo A. 2016. - European Red List of Habitats - Marine: Mediterranean Sea Habitat Group. A2.13 Communities of Mediterranean Mediolittoral Coarse Sediment. European Union, Luxembourg, 9 p.

Sordello R., Conruyt-Rogeon G., Merlet F., Houard X. \& TOUROULT J. 2013. - Synthèses bibliographiques sur les traits de vie de 39 espèces proposées pour la cohérence nationale de la Trame verte et bleue relatifs à leurs déplacements et besoins de continuité écologique. MNHN-SPN; OPIE, Paris, 20 p. + 39 fiches.

Soustelle C., Moisset F. \& Lereec Le BricQuir M.-L. 2019. Première mention documentée de Pantala flavescens en France métropolitaine (Odonata: Libellulidae). Martinia 34: 61-67.

ŠPALDOŇOVÁ A. \& FrOUZ J. 2014. - The role of Armadillidium vulgare (Isopoda: Oniscidea) in litter decomposition and soil organic matter stabilization. Applied Soil Ecology 83: 186-192. https://doi.org/10.1016/j.apsoil.2014.04.012

StallegGer P. (coord.) 2019. - Atlas des Orthoptères de Normandie. Invertébrés armoricains 19: 1-226.

STETTMER C. 1996. - Colonisation and dispersal patterns of banded (Calopteryx splendens) and beautiful demoiselles (C. virgo) (Odonata: Calopterygidae) in south-east German streams. European Journal of Entomology 93 (4): 579-593.

TAITI S. \& FERRERA F. 1996. - The terrestrial Isopoda of Corsica (Crustacea, Oniscidea). Bulletin du Muséum national d'Histoire naturelle 18 (3-4): 459-545.

Tassi F., Aberlenc H.-P., Rasplus J.-Y., Curletti G., Dutto M., Genson G. \& LeMPÉRIÈre G. 2004. - Eupotosia mirifica, la Grande Cétoine bleue, joyau menacé du patrimoine naturel européen. Propositions pour la protection de l'espèce et de ses biotopes (Coleoptera Cetoniidae Cetoniinae). Lambillionea 104 (1) Suppl.: $1-32$.

TAUZIN P.-H. 2004. — Quelques localités connues pour les espèces françaises d'Aleurostictus (Coleoptera, Cetoniidae, Trichiinae, Trichiini). Cetoniimania 1 (1): 33-52.

Tauzin P.-H. 2007. - Chorologie de Protaetia (Potosia) opaca Fabricius 1787 en France (Coleoptera, Cetoniinae, Cetoniini). Cetoniimania 4 (1-2): 19-48.

Thomas H. 2014. - Note sur la répartition en France et quelques éléments d'écologie de Phaleria atlantica Fauvel, 1899 (Coleoptera, Tenebrionidae). Bulletin de la Société linnéenne de Bordeaux 149 (N. S.), 42 (2): 159-162. 
TOLSGAARD S. 1998. — Bredtæger og randtæger, in STOLTZE M. \& Pinl S. (éds), Rødliste 1997 over planter og dyr i Danmark. Miljøog Energiministeriet, Danmarks Miljøundersøgelser og Skov- og Naturstyrelsen, København: 94-96.

TouroulT J. 2014. - Les inventaires de répartition, une approche dépassée? Mémoires de la Société entomologique de France 9: 67-76.

Touroult J., Cima V., Bouyon H., Hanot C., Horellou A. \& BRUSTEL H. 2019. - Longicornes de France-Atlas préliminaire (Coleoptera: Cerambycidae \& Vesperidae). Supplément au bulletin d'ACOREP-France, Paris, $176 \mathrm{p}$.

Touroult J., Poncet L., Keith P., Boullet V., Arnal G., BrusTEL H. \& SibleT J.-P. 2015. - Inventaires et atlas nationaux de distribution : pour une approche plus itérative et un rééquilibrage taxinomique. Revue d'Ecologie (Terre et Vie) 70 (2): 97-120.

UICN FRANCE 2016. - La Liste rouge des écosystèmes en France. Habitats forestiers de France métropolitaine. Recueil des études de cas. UICN France, Paris, $94 \mathrm{p}$.

UICN FRANCE 2018a. - Guide pratique pour la réalisation de Listes rouges régionales des espèces menacées - Méthodologie de l'UICN \& démarche d'élaboration. Seconde édition. UICN France, Paris, 48 p. + Annexes.

UICN FrANCE 2018b. - La Liste rouge des Écosystèmes en FranceChapitre Forêts méditerranéennes de France métropolitaine. UICN France, Paris, 27 p.

UICN FRANCE 2020. - La Liste rouge des Écosystèmes en France Chapitre Littoraux méditerranéens de France métropolitaine. Vol. 1 : dunes côtières et rivages sableux. UICN France, Paris, $28 \mathrm{p}$.

UICN FRANCE \& MNHN 2012. - La Liste rouge des espèces menacées en France. Papillons de jour de France métropolitaine. UICN, Opie, Société entomologique de France, Paris, 18 p.

UICN FRANCE, MNHN \& OPIE 2018. — La Liste rouge des espèces menacées en France. Éphémères de France métropolitaine. UICN France, MNHN, OPIE, Paris, $4 \mathrm{p}$.

UICN FranCE, MNHN, SFEPM \& ONCFS. 2009. — La liste rouge des espèces menacées en France. Chapitre Mammiferes de France métropolitaine. UICN France, MNHN, SFEPM, ONCFS, Paris, $12 \mathrm{p}$.

UICN FRANCE, MNHN, OPIE \& SFO 2016. — La Liste rouge des espèces menacées en France - Chapitre Libellules de France métropolitaine. UICN France, MNHN, OPIE, SFO, Paris, 11 p.

Van SwaAy C., CutTelod A., Collins S., Maes D., López Munguira M., Šašić M., Settele J., Verovinik R., Verstrael T.,
WARren M., Wiemers M. \& Wynhof I. 2010. - European Red List of Butterflies. Publications Office of the European Union, Luxembourg, 26 p. + Annexes.

Vanappelghem C., Vandeweghe R., Debaive N., Claude J. Dussaix C., Garrigue J., Gaudet S., Langlois D., MailLet G., Sarthou V., Sarthou J.-P., Soissons A., Speight M., Tissot B., Top D., Tourdiat S. \& Vallet A. 2020. — Guide technique de mise en ouvre d'une étude Syrph the Net. Retours d'expérience de l'atelier du groupe inter-réseaux Syrphes. Réserves naturelles de France, Dijon, 121 p.

Villalobos-Jiménez, DunN A. M. \& Hassall C. 2016. — Dragonflies and damselflies (Odonata) in urban ecosystems: a review. European Journal of Entomology 113: 217-232. https://doi. org/10.14411/eje.2016.027

Villiers A. 1978. - Faune des Coléoptères de France. I. Cerambycidae. Encyclopédie Entomologique, XLII. Lechevalier, Paris, 611 p.

Vitali F. \& SCHMitT T. 2016. - Ecological patterns strongly impact the biogeography of western Palaearctic longhorn beetles (Coleoptera: Cerambycoidea). Organisms Diversity \& Evolution 17 (1): 163-180. https://doi.org/10.1007/s13127-016-0290-6

Voigtländer K. 2011. - Chilopoda - Ecology, in Minelli A. (éd.), Treatise on Zoology - Anatomy, Taxonomy, Biology. The Myriapoda, Vol. I. Brill: 309-325.

VOISIN J.-F. (coord.) 2003. - Atlas des Orthoptères (Insecta: Orthoptera) et des Mantides (Insecta: Mantodea) de France. Patrimoines Naturels 60: $104 \mathrm{p}$.

WallisDeVries M. F. \& VAN SWAay C. A. M. 2009. — Grasslands as habitats for butterflies in Europe, in VEEN P., JEFFERSON R., DE SCMidT J. \& VAN DER STRAATEN J. (éds), Grasslands in Europe of High Nature Value. KNNV Uitgeverij: 27-34. https:// doi.org/10.1163/9789004278103_004

Warren M. S., Maes D., Van SwaAy C. A. M., Goffart P., Van Dyck H., Bourn N. A. D., Wynhoff I., HoAre D. \& Ellis S. 2021. - The decline of butterflies in Europe: problems, significance, and possible solutions. Proceedings of the National Academy of Sciences of the United States of America 118 (2): 1-10. https:// doi.org/10.1073/pnas.2002551117

Zimmer M., Pennings S. C., Buck T. L. \& Carefoot T. H. 2002. - Species-specific patterns of litter processing by terrestrial isopods (Isopoda: Oniscidea) in high intertidal salt marshes and coastal forests. Functional Ecology 16: 596-607. https://doi. org/10.1046/j.1365-2435.2002.00669.x 


\section{ANNEXES}

ANNEXE 1. - Liste des espèces autochtones d'Arthropodes terrestres à statut réglementaire au(x) niveau(x) national et/ou européen en France (TaxRef V14 et base de connaissance statut). Abréviations: BE2, Annexe 2 de la Convention de Berne; BE4, Annexe 4 de la Convention de Berne; DH2, espèce inscrite à l'annexe 2 de la Directive Habitat-Faune-Flore (Directive 92/43/CEE du 21 mai 1992 modifiée par la Directive 97/62/CEE); DH4, idem, annexe 4; DH5, idem, annexe 5; PN2, arrêté du 23 avril 2007 fixant les listes d'Insectes protégés sur l'ensemble du territoire métropolitain, article 2; PN3, idem, article 3. Le terme d'espèces «réglementées" parfois utilisé dans le texte de notre article inclue les espèces protégées au sens strict (PN2, PN3, DH4) et celles uniquement inscrites à l'annexe 2 de la DHFF. *, sur l'INPN, toutes les espèces comprises dans le genre Aphaenops s. I. apparaissent comme protégées (50 espèces en tout). Si l'on se fie à l'Arrêté du 23 avril 2007, seules les espèces réparties dans les sous-genres Aphaenops Bonvouloir, 1862 et Hydraphaenops Jeannel, 1916 seraient concernées (aucun taxon de rang spécifique n'y étant précisé pour ceux-ci).

\begin{tabular}{|c|c|c|}
\hline Ordre & Espèce & Type(s) de protection(s) \\
\hline Odonates & $\begin{array}{l}\text { Coenagrion mercuriale (Charpentier, 1840) } \\
\text { Coenagrion ornatum (Selys, 1850) } \\
\text { Gomphus graslinii Rambur, 1842 } \\
\text { Leucorrhinia albifrons (Burmeister, 1839) } \\
\text { Leucorrhinia caudalis (Charpentier, 1840) } \\
\text { Leucorrhinia pectoralis (Charpentier, 1825) } \\
\text { Lindenia tetraphylla (Vander Linden, 1825) } \\
\text { Macromia splendens (Pictet, 1843) } \\
\text { Ophiogomphus cecilia (Geoffroy in Fourcroy, 1785) } \\
\text { Oxygastra curtisii (Dale, 1834) } \\
\text { Stylurus flavipes (= Gomphus flavipes) (Charpentier, 1825) } \\
\text { Sympecma paedisca (Brauer, 1877) }\end{array}$ & $\begin{array}{l}\mathrm{PN} 3, \mathrm{DH} 2 \\
\mathrm{DH} 2 \\
\mathrm{PN} 2, \mathrm{BE} 2, \mathrm{DH} 2, \mathrm{DH} 4 \\
\mathrm{PN} 2, \mathrm{DH} 4 \\
\mathrm{PN} 2, \mathrm{DH} 4 \\
\mathrm{PN} 2, \mathrm{DH} 2, \mathrm{DH} 4 \\
\mathrm{BE} 2, \mathrm{DH} 2, \mathrm{DH} 4 \\
\mathrm{PN} 2, \mathrm{DH} 2, \mathrm{DH} 4 \\
\mathrm{PN} 2, \mathrm{DH} 2, \mathrm{DH} 4 \\
\mathrm{PN} 2, \mathrm{DH} 2, \mathrm{DH} 4 \\
\mathrm{PN} 2, \mathrm{DH} 4 \\
\mathrm{PN} 2, \mathrm{BE} 2, \mathrm{DH} 4\end{array}$ \\
\hline Orthoptères & $\begin{array}{l}\text { Saga pedo (Pallas, 1771) } \\
\text { Prionotropis azami Uvarov, } 1923 \\
\text { Prionotropis rhodanica Uvarov, } 1923\end{array}$ & $\begin{array}{l}\text { PN2, DH4 } \\
\text { PN3 } \\
\text { PN3 }\end{array}$ \\
\hline Coléoptères & $\begin{array}{l}\text { Aphaenops spp. Bonvouloir, } 1862 \text { (50 espèces)* } \\
\text { Bolbelasmus unicornis Schranck, } 1789 \\
\text { Carabus auratus honnoratii Dejean, } 1826 \\
\text { Carabus auronitens cupreonitens Chevrolat, } 1861 \\
\text { Carabus auronitens subfestivus Oberthür, } 1884 \\
\text { Carabus nodulosus (= Carabus variolosus) Creutzer, } 1799 \\
\text { Carabus solieri Dejean, } 1826 \\
\text { Cerambyx cerdo Linnaeus, } 1758 \\
\text { Cucujus cinnaberinus (Scopoli, 1763) } \\
\text { Dytiscus latissimus Linnaeus, 1758 } \\
\text { Graphoderus bilineatus (de Geer, 1774) } \\
\text { Limoniscus violaceus (P.W.J. Müller, 1821) } \\
\text { Lucanus cervus (Linnaeus, 1758) } \\
\text { Osmoderma eremita (Scopoli, 1763) } \\
\text { Phryganophilus ruficollis (Fabricius, 1798) } \\
\text { Rhysodes sulcatus (Fabricius, 1787) } \\
\text { Rosalia alpina (Linnaeus, 1758) } \\
\text { Stephanopachys linearis (Kugelann, 1792) } \\
\text { Stephanopachys substriatus (Paykull, 1800) } \\
\text { Trichaphaenops spp. Jeannel, 1916 (quatre espèces) }\end{array}$ & $\begin{array}{l}\text { PN3 } \\
\text { PN2, DH2, DH4 } \\
\text { PN3 } \\
\text { PN3 } \\
\text { PN3 } \\
\text { PN2 } \\
\text { PN3 } \\
\text { PN2, BE2, DH2, DH4 } \\
\text { PN2, BE2, DH2, DH4 } \\
\text { PN2, BE2, DH2, DH4 } \\
\text { PN2, BE2, DH2, DH4 } \\
\text { DH2 } \\
\text { BE3, DH2 } \\
\text { PN2, BE2, DH2, DH4 } \\
\text { PN2, DH2, DH4 } \\
\text { DH2 } \\
\text { PN2, BE2, DH2, DH4 } \\
\text { DH2 } \\
\text { DH2 } \\
\text { PN3 }\end{array}$ \\
\hline Lépidoptères & $\begin{array}{l}\text { Arctia matronula (= Pericallia matronula) (Linnaeus, 1758) } \\
\text { Boloria aquilonaris (Stichel, 1908) } \\
\text { Boloria eunomia (= Proclossiana eunomia) (Esper, 1800) } \\
\text { Coenonympha hero (Linnaeus, 1760) } \\
\text { Coenonympha oedippus (Fabricus, 1787) } \\
\text { Coenonympha tullia (O.F. Müller, 1764) } \\
\text { Colias palaeno (Linnaeus, 1760) } \\
\text { Diacrisia metelkana (Lederer, 1861) } \\
\text { Epatolmis luctifera (= Phragmatobia caesarea) (Denis \& Schiffermüller, 1775) } \\
\text { Erebia sudetica Staudinger, 1861 } \\
\text { Eriogaster catax (Linnaeus, 1758) } \\
\text { Euphydryas aurinia (Rottemburg, 1775) } \\
\text { Euphydryas desfontainii (Godart, 1819) } \\
\text { Euphydryas maturna (= Hypodryas maturna) (Linnaeus, 1758) } \\
\text { Euplagia quadripunctaria (Poda, 1761) } \\
\text { Fabriciana elisa (Godart, 1823) } \\
\text { Gortyna borelii Pierret, 1838 } \\
\text { Graellsia isabellae (Graells, 1849) } \\
\text { Hyles hippophaes (Esper, 1789) } \\
\text { Lopinga achine (Scopoli, 1763) } \\
\text { Lycaena dispar (Haworth, 1803) } \\
\text { Lycaena helle (Denis et Schiffermuller, 1775) } \\
\text { Papilio alexanor Esper, 1800 } \\
\text { Papilio hospiton Genè, 1839 }\end{array}$ & $\begin{array}{l}\text { PN3 } \\
\text { PN3 } \\
\text { PN3 } \\
\text { PN2, BE2, DH4 } \\
\text { PN2, BE2, DH2, DH4 } \\
\text { PN3 } \\
\text { PN3 } \\
\text { PN3 } \\
\text { PN3 } \\
\text { PN2, BE2, DH4 } \\
\text { PN2, BE2, DH2, DH4 } \\
\text { PN3, BE2, DH2 } \\
\text { PN3 } \\
\text { PN2, BE2, DH2, DH4 } \\
\text { DH2 } \\
\text { PN2, BE2, DH4 } \\
\text { PN2, DH2, DH4 } \\
\text { PN3, BE3, DH2, DH5 } \\
\text { PN2, BE2, DH4 } \\
\text { PN2, BE2, DH4 } \\
\text { PN2, BE2, DH2, DH4 } \\
\text { PN2, DH2, DH4 } \\
\text { PN2, BE2, DH4 } \\
\text { PN2, BE2, DH2 }\end{array}$ \\
\hline
\end{tabular}


AnNeXe 1. - Suite.

\begin{tabular}{ll}
\hline Espèce & Type(s) de protection(s) \\
\hline Parnassius apollo (Linnaeus, 1758) & PN2, BE2, DH4 \\
Parnassius corybas (= Parnassius phoebus) Fischer de Waldheim, 1823 & PN3 \\
Parnassius mnemosyne (Linnaeus, 1758) & PN2, BE2, DH4 \\
Phengaris alcon (= Maculinea alcon) (Denis et Schiffermuller, 1775) & PN3, BE2 \\
Phengaris arion (= Maculinea arion) (Linnaeus, 1758) & PN2, BE2, DH4 \\
Phengaris nausithous (= Maculinea nausithous) (Bergstrasser, 1779) & PN2, BE2, DH2, DH4 \\
Phengaris teleius (= Maculinea telejus) (Bergstrasser, 1779) & PN2, DH2, DH4 \\
Pieris ergane (Geyer, 1828) & PN3 \\
Proserpinus proserpina Pallas, 1772 & PN2, BE2, DH4 \\
Zerynthia polyxena (Denis et Schiffermuller, 1775) & PN2, BE2, DH4 \\
Zerynthia rumina (Linnaeus, 1758) & PN3 \\
Zygaena rhadamanthus (Esper, 1789) & PN3 \\
Zygaena brizae vesubiana Le Charles, 1933 & PN3 \\
\hline
\end{tabular}


ANNEXE 2. - Sélection d'espèces à enjeux remarquables pour les neuf groupes étudiés. Abréviations: A, habitats marins; $\mathbf{B}$, habitats côtiers; C, eaux de surface continentales; D, tourbières hautes et bas-marais; E, prairies, terrains dominés par des herbacées non graminoïdes, des mousses ou des lichens; $\mathbf{F}$, landes, fourrés et toundras; G, boisements, forêts et autres habitats boisés; $\mathbf{H}$, habitats continentaux sans végétation ou à végétation clairsemée; I, habitats agricoles, horticoles et domestiques régulièrement ou récemment cultivés; INPN, inventaire national du patrimoine naturel; $\mathbf{J}$, zones bâties, sites industriels et autres habitats artificiels; PACA, Provence-Alpes-Côte d'Azur; ZNIEFF, zone naturelle d'intérêt écologique, faunistique et floristique.

\begin{tabular}{|c|c|c|}
\hline \multicolumn{2}{|c|}{ Espèces à enjeux remarquables } & Commentaire \\
\hline Chilopodes & $\begin{array}{l}\text { Clinopodes vesubiensis } \\
\text { Bonato, lorio \& Minelli, } \\
2011\end{array}$ & $\begin{array}{l}\text { Endémique très localisé: vallée de la Vésubie, en étage montagnard } \\
\text { et subalpin. Un des rares Chilopodes spécialiste des milieux } \\
\text { ouverts et semi-ouverts pierreux de ce secteur. Espèce jugée } \\
\text { «vulnérable»(Annexe 3). Déterminant pour les ZNIEFF de PACA } \\
\text { (source: INPN). Seule espèce française du genre Clinopodes (Bonato }\end{array}$ \\
\hline
\end{tabular}

Cryptops lobatus Verhoeff, 1931

Cryptops umbricus Verhoeff, 1931

Eupolybothrus tridentinus (Fanzago, 1874)

Geophilus persephones Foddai \& Minelli, 1999

Henia bicarinata (Meinert, 1870)

Lithobius cavernicolus Fanzago, 1877

Lithobius dentatus

C. L. Koch, 1844

Lithobius fagniezi Ribaut, 1926

Lithobius pelidnus Haase, 1880

Lithobius raffaldii lorio, 2009 et al. 2011).
Répartition très fragmentée: bastion allant de la Ligurie à l'Est du Var et petite zone déconnectée côté espagnol. Sylvicole. Espèce «vulnérable» (Annexe 3), déjà jugée sensible (Geoffroy \& lorio 2019). Déterminant pour les ZNIEFF de PACA (source: INPN). Identification assez simple sous loupe binoculaire (lorio \& Geoffroy 2008; lorio 2010a).

Subendémique (France-Italie) cavernicole à répartition fragmentée, présent dans les Alpes-Maritimes, les Alpes-de-Haute-Provence et l'extrême est du Var. Déterminant pour les ZNIEFF de PACA (source: INPN). Grand (35-50 mm), se distinguant sans peine avec ses antennes très longues (lorio \& Geoffroy 2008), même sur photos. Inclut deux sous-espèces chez nous dont C. umbricus lewisi lorio, 2010 (endémique).

Répartition centre-européenne peu étendue et fragmentée. Rarissime en France, où il ne vit que dans les forêts caducifoliées ou mixtes montagnardes dominées par le Hêtre. Facile à identifier sous loupe binoculaire en étant initié (lorio \& Labroche 2015; lorio et al. 2019). «Parapluie» pour le très rare Lithobius mutabilis L. Koch, 1862.

Géophilomorphe troglobie micro-endémique du gouffre de la Pierre-SaintMartin (64). Il avait déjà été défini comme un enjeu majeur (lorio 2014). Menacé, jugé «vulnérable» (Annexe 3). Son identification s'appuie sur Foddai \& Minelli (1999).

Halophile des plages peu perturbées de la Méditerranée, avant tout sous les galets en contact étroit avec un substrat plus fin, aussi dans les laisses de mer. Habitat très étroit, $<5$ mètres de large. Très menacée, jugée «en danger d'extinction » (Annexe 3). Déterminante pour les ZNIEFF de PACA (source: INPN). Facile à chercher et très aisée à identifier à vue ou sur macrophoto pour l'initié (voir fiche de lorio et al. [2020]). Bonne espèce «parapluie» pour Geophilus fucorum Brölemann, 1909, autre Chilopode menacé, ainsi que pour les autres halophiles du bord de mer (lorio et al. 2020).

Espèce troglobie quasi-endémique d'Ariège, à répartition fragmentée. L'identification se fait sous la loupe binoculaire. Avec un peu d'expérience, elle est de difficulté modérée (lorio 2010b).

Répartition centre-européenne, morcelée en France. Rare. Typique des forêts planitiaires et collinéennes caducifoliées ou mixtes fraîches, humides et peu perturbées dans le Nord; des forêts de l'étage montagnard dans le Sud-Est où elle est encore plus rare. Identification accessible avec lorio \& Labroche (2015) et lorio (2010b).

Troglobionte endémique du Sud-Ouest du Var, qui ne vit plus que dans six grottes aujourd'hui. Fortement menacé, jugé «en danger d'extinction» (Annexe 3). Déterminant pour les ZNIEFF de PACA (source: INPN). Assez aisé à reconnaître sous la loupe voire sur macrophotos par un initié avec sa localisation, son habitat et ses principaux caractères (lorio 2010b).

Répartition surtout centre-européenne, morcelée en France. Rare. Quasi-exclusif des grands massifs forestiers planitiaires et collinéens caducifoliés et mixtes frais et humides, peu perturbés. Déterminant pour les ZNIEFF de Lorraine (source: INPN). Bon «parapluie» pour d'autres Chilopodes forestiers exigeants (e.g. Lithobius aeruginosus L. Koch, 1862). L'identification n'est que peu difficile pour les mâles (Iorio \& Labroche 2015).

Troglobionte endémique très localisé des grottes de Haute-Corse. Jugé «vulnérable» (Annexe 3). Il constitue un «parapluie» pour d'autres cavernicoles, dont le micro-endémique Lithobius cherpinedensis lorio, 2010 avec qui il partage sa station-type. L. raffaldii est grand (25$32 \mathrm{~mm}$ ) et facile à reconnaître (lorio 2010b).

EUNIS

Niveau

E, F Majeur

G Fort

Fort

Fort

A, B Majeur

Fort

H Majeur

Fort

$\mathrm{H}$

Majeur 


\begin{tabular}{|c|c|c|c|c|}
\hline \multicolumn{2}{|c|}{ Espèces à enjeux remarquables } & \multirow{2}{*}{$\begin{array}{l}\text { Commentaire } \\
\text { Endémique pyrénéen, très rare, à répartition fragmentée. Forêts } \\
\text { submontagnardes à montagnardes, privilégiant les terrains moussus; } \\
\text { parfois dans l'entrée des grottes se trouvant en contexte forestier. } \\
\text { Reconnaissable en un clin d'œil sous stéréomicroscope avec un peu } \\
\text { de pratique, car doté de forcipules uniques en France. }\end{array}$} & \multirow{2}{*}{$\begin{array}{r}\text { EUNIS } \\
\text { G, H }\end{array}$} & \multirow{2}{*}{$\begin{array}{c}\begin{array}{c}\text { Niveau } \\
\text { d'enjeu }\end{array} \\
\text { Fort }\end{array}$} \\
\hline & $\begin{array}{l}\text { Lithobius ribauti } \\
\text { Chalande, } 1907\end{array}$ & & & \\
\hline & $\begin{array}{l}\text { Lithobius variegatus } \\
\quad \text { Leach, } 1814\end{array}$ & $\begin{array}{l}\text { Répartition très fragmentée en France et limitée aux régions atlantiques; } \\
\text { très rare. Forêts caducifoliées peu perturbées. Menacé, jugé } \\
\text { «vulnérable» (Annexe 3), réduction de l'aire d'occurrence > } 50 \% \text { en } \\
\text { France. Déterminant pour les ZNIEFF de deux régions: Bretagne et } \\
\text { Pays de la Loire (source: INPN). Facile à identifier à vue ou sur photo } \\
\text { (lorio \& Labroche 2015). }\end{array}$ & G & Majeur \\
\hline & $\begin{array}{l}\text { Schendyla monodi } \\
\quad \text { (Brolemann, 1924) }\end{array}$ & $\begin{array}{l}\text { Endémique de France, spécialiste du schorre et de la haute slikke de } \\
\text { l'Atlantique. Très rare et localisée, répartition fragmentée. Menacée, } \\
\text { jugée «vulnérable» (Annexe 3). Déterminante pour les ZNIEFF de } \\
\text { Bretagne (source: INPN). Identification sous la loupe binoculaire } \\
\text { minutieuse, mais facilitée par lorio \& Labroche (2015). }\end{array}$ & A & Majeur \\
\hline & $\begin{array}{l}\text { Tuoba poseidonis } \\
\text { (Verhoeff, 1901) }\end{array}$ & $\begin{array}{l}\text { Même cas de figure qu'H. bicarinata mais encore plus spécialisé. Mobilité } \\
\text { et milieu de vie extrêmement restreints, entre } 0,7 \text { et } 3 \text { m de distance } \\
\text { de la ligne d'eau. Très sensible: le surpiétinement des plages est à lui } \\
\text { seul antagoniste, même si les autres conditions demeurent favorables. } \\
\text { Elle peut être pressentie à vue, un bref passage sous loupe binoculaire } \\
\text { achevant l'identification (voir fiche lorio et al. 2020). Facile à chercher. }\end{array}$ & $A, B$ & Majeur \\
\hline \multirow[t]{6}{*}{$\begin{array}{l}\text { Isopodes } \\
\text { terrestres }\end{array}$} & $\begin{array}{l}\text { Alloschizidium spp } \\
\text { Verhoeff, } 1919\end{array}$ & $\begin{array}{l}\text { Cinq espèces, endémiques de France ou corso-sardes, assez ou très } \\
\text { localisées. Distribution relictuelle, faible capacité de dispersion. } \\
\text { Espèces regroupées en un taxon générique pour simplifier } \\
\text { l'identification par ce moyen, sachant que les cinq espèces sont } \\
\text { d'enjeu majeur. Espèces toutes endogées, liées à un habitat ou micro- } \\
\text { habitat rare en contexte forestier: } \\
\text { - A. campanellii Taiti \& Ferrara, 1996: boisements et fourrés } \\
\text { sempervirents à Chêne vert et/ou à Arbousier; } \\
\text { - A. cottarellii (Argano \& Pesce, 1974): myrmécophile, mentions de } \\
\text { boisements et fourrés à Chêne vert et/ou à Arbousier; } \\
\text { - A. pruvoti (Racovitza, 1907): endogée et cavernicole; } \\
\text { - A. racovitzai Vandel, 1954: endogée, sous les grosses pierres } \\
\text { enfoncées, dans des terrains plantés de chênes, rarement dans les } \\
\text { grottes; } \\
\text { - A. remyi (Vandel, 1944): a priori comme A. racovitzai (Séchet \& Noël } \\
\text { 2015). }\end{array}$ & $\mathrm{G}$ & Majeur \\
\hline & $\begin{array}{l}\text { Armadillidium album } \\
\text { Dollfus, } 1887\end{array}$ & $\begin{array}{l}\text { Surtout Manche et Atlantique (très rare en Méditerranée). Large } \\
\text { répartition, mais très fragmentée. Espèce halophile supralittorale des } \\
\text { plages, liée à un milieu rare, principalement les laisses de mer (Séchet } \\
\text { \& Noël 2015). Faible capacité de dispersion. En déclin formellement } \\
\text { avéré, ou déduit du déclin des habitats. }\end{array}$ & $\mathrm{B}$ & Fort \\
\hline & $\begin{array}{c}\text { Buchnerillo litoralis } \\
\text { Verhoeff, } 1943\end{array}$ & $\begin{array}{l}\text { Espèce présente sur le littoral de la Méditerranée et de l'Atlantique, aussi } \\
\text { sur les îles comme Malte et Madère. Halophile à large répartition, mais } \\
\text { très fragmentée; liée à un micro-habitat rare (principalement dans les } \\
\text { mattes anciennes de posidonie). Faible capacité de dispersion. }\end{array}$ & $\mathrm{B}$ & Fort \\
\hline & $\begin{array}{l}\text { Buddelundiella zimmeri } \\
\text { Verhoeff, } 1930\end{array}$ & $\begin{array}{l}\text { Espèce tyrrhénienne (Italie, Sud-Est de la France \& Monaco) = sub- } \\
\text { endémique plus large. Faible capacité de dispersion. Grottes } \\
\text { uniquement. http://endemica.mnhn.fr/Species/Buddelundiella.zimmeri. } \\
\text { zimmeri.html, dernière consultation le } 10 \text { janvier } 2021 .\end{array}$ & $\mathrm{H}$ & Fort \\
\hline & $\begin{array}{l}\text { Cyrnoniscus remyi } \\
\text { Vandel, } 1953\end{array}$ & $\begin{array}{l}\text { Espèce endémique très localisée (< } 10 \text { stations) propre à la Corse. Faible } \\
\text { capacité de dispersion. Grottes uniquement. http://endemica.mnhn. } \\
\text { fr/Species/Cyphotendana.dalmazzensis.html, dernière consultation le } \\
10 \text { janvier } 2021 .\end{array}$ & $\mathrm{H}$ & Majeur \\
\hline & $\begin{array}{l}\text { Escualdoniscus spp } \\
\quad \text { (Vandel, 1948) }\end{array}$ & $\begin{array}{l}\text { Deux espèces endogées/cavernicoles localisées au Pays Basque } \\
\text { (subendémiques franco-espagnoles): Escualdoniscus coiffaiti (Vandel, } \\
\text { 1948) et E. triocellatus (Vandel, 1948). Moins de cinq stations connues au } \\
\text { total. Endémiques très localisées pour chaque espèce et faible capacité de } \\
\text { dispersion. Espèces regroupées en un taxon générique car simplification } \\
\text { de l'identification par ce moyen, sachant que les deux espèces sont } \\
\text { d'enjeu majeur. http://endemica.mnhn.fr/Species/Escualdoniscus.coiffaiti. } \\
\text { html et http://endemica.mnhn.fr/Species/Escualdoniscus.triocellatus.html, } \\
\text { dernières consultations le } 10 \text { janvier 2021. }\end{array}$ & 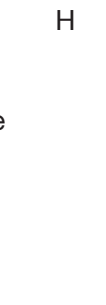 & Majeur \\
\hline
\end{tabular}




\begin{tabular}{|c|c|c|c|c|}
\hline Espèces à enjeu & ux remarquables & Commentaire & EUNIS & $\begin{array}{l}\text { Niveau } \\
\text { d'enjeu }\end{array}$ \\
\hline & \multicolumn{2}{|c|}{$\begin{array}{l}\text { Phymatoniscus propinquus Cavernicole endémique plus large (Hérault, Gard, Ardèche, Vaucluse). } \\
\begin{array}{l}\text { (Carl, 1908) } \\
\text { Faible capacité de dispersion. Une trentaine de stations connues en } \\
\text { France (grottes) (Séchet \& Noël 2015). }\end{array}\end{array}$} & $\mathrm{H}$ & Fort \\
\hline & $\begin{array}{l}\text { Porcellio alticola } \\
\quad \text { Vandel, } 1951\end{array}$ & $\begin{array}{l}\text { Endémique très localisé propre aux Pyrénées-Orientales. Montagnarde, } \\
\text { vivant dans les prairies jusqu'à } 2000 \text { m d'altitude. Faible capacité de } \\
\text { dispersion. }\end{array}$ & $\mathrm{E}$ & Majeur \\
\hline & $\begin{array}{l}\text { Porcellio provincialis } \\
\quad \text { Aubert \& Dollfus, } 1890\end{array}$ & $\begin{array}{l}\text { Endémique du Sud-Est de la France (Var, Bouches-du-Rhône, Hautes- } \\
\text { Alpes). Répartition très fragmentée. Faible capacité de dispersion. Espèce } \\
\text { liée à un milieu rare: propre aux collines calcaires et sèches de la Provence } \\
\text { et des Préalpes crétacées. http://endemica.mnhn.fr/Species/Porcellio. } \\
\text { provincialis.html, dernière consultation le } 10 \text { janvier } 2021 .\end{array}$ & 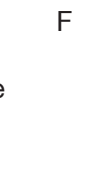 & Majeur \\
\hline & $\begin{array}{l}\text { Scotoniscus macromelos } \\
\quad \text { Racovitza, } 1908\end{array}$ & $\begin{array}{l}\text { Endémique plus large, espèce relictuelle des Pyrénées françaises, } \\
\text { cavernicole se découpant en neuf sous-espèces localisées (Séchet \& } \\
\text { Noël 2015). }\end{array}$ & $\mathrm{H}$ & Fort \\
\hline & $\begin{array}{l}\text { Tendosphaera verrucosa } \\
\text { Verhoeff, } 1930\end{array}$ & $\begin{array}{l}\text { Subendémique des Alpes franco-italiennes. Faible capacité de dispersion. } \\
\text { Monticole propre aux forêts d'altitude. }\end{array}$ & G & Fort \\
\hline & $\begin{array}{l}\text { Tiroloscia esterelana } \\
\quad \text { (Verhoeff, 1918) }\end{array}$ & $\begin{array}{l}\text { Endémique plus large, présente dans le Sud-Est de la France (Esterel et } \\
\text { Maures). Faible capacité de dispersion. Sylvicole, généralement dans } \\
\text { les châtaigneraies et les chênaies vertes. }\end{array}$ & $\mathrm{G}$ & Fort \\
\hline & $\begin{array}{l}\text { Tiroloscia montana } \\
\quad \text { (Taiti \& Ferrara, 1996) }\end{array}$ & $\begin{array}{l}\text { Endémique très localisée, propre à la Corse. Distribution relictuelle. } \\
\text { Espèce monticole, vivant dans les montagnes d'Asco à plus de } 1500 \text { m } \\
\text { d'altitude. http://endemica.mnhn.fr/Species/Tiroloscia.montana.html, } \\
\text { dernière consultation le } 10 \text { janvier } 2021 \text {. }\end{array}$ & G & Majeur \\
\hline & $\begin{array}{l}\text { Trichoniscus halophilus } \\
\quad \text { Vandel, } 1951\end{array}$ & $\begin{array}{l}\text { Méditerranée et mer lonnienne, répartition large mais très fragmentée. } \\
\text { Espèce littorale halophile, uniquement présente sur les îles en France. } \\
\text { En déclin formellement avéré, ou déduit du déclin des habitats } \\
\text { en raison de la forte pression anthropique sur ces derniers. Large } \\
\text { répartition mais espèce liée à un micro-habitat rare (généralement dans } \\
\text { les débris végétaux de type posidonies échouées ou tiges de Canne de } \\
\text { Provence, sous les morceaux de bois, etc.). }\end{array}$ & (1) & Majeur \\
\hline & Tylos spp. Audouin, 1826 & $\begin{array}{l}\text { Deux espèces littorales, halophiles, à large répartition, mais très } \\
\text { fragmentée. Espèces liées à un milieu rare, présentent sur l'étage } \\
\text { supralittoral des plages mais aussi dans le médiolittoral supérieur. } \\
\text { Espèces regroupées en un taxon générique car simplification de } \\
\text { l'identification par ce moyen, sachant que les deux espèces sont d'enjeu } \\
\text { fort (Tylos europaeus Linnaeus, } 1758 \text { et } T \text {. ponticus Grebnitzky, 1874). }\end{array}$ & $A, B$ & Fort \\
\hline \multirow[t]{6}{*}{$\begin{array}{l}\text { Coléoptères } \\
\text { Longicornes } \\
\text { (Cerambycidae } \\
\text { et Vesperidae) }\end{array}$} & $\begin{array}{l}\text { Akimerus schaefferi } \\
\text { (Laicharting, 1784) }\end{array}$ & $\begin{array}{l}\text { Espèce à vaste aire de répartition mais toujours localisée. En France, très } \\
\text { localisée à quelques grandes chênaies anciennes réparties sur toute la } \\
\text { France (Touroult et al. 2019). Espèce déterminante ZNIEFF dans quatre } \\
\text { régions (source: INPN) et classée «en danger » dans la Liste rouge } \\
\text { européenne (Cálix et al. 2018). }\end{array}$ & G & Majeur \\
\hline & $\begin{array}{l}\text { Brachyta borni } \\
\text { (Ganglbauer, 1903) }\end{array}$ & $\begin{array}{l}\text { Endémique stricte d'une petite zone des Alpes françaises, dans des } \\
\text { milieux prairiaux d'altitude. Facilement détectable. Pas de menace } \\
\text { directe actuelle mais potentiellement sensible à tout aménagement de } \\
\text { ses quelques sites de présence. }\end{array}$ & $\mathrm{E}$ & Majeur \\
\hline & $\begin{array}{l}\text { Cerambyx welensii } \\
\quad \text { (Küster, 1845) }\end{array}$ & $\begin{array}{l}\text { Espèce compagne de Cerambyx cerdo Linnaeus, 1758, espèce déjà } \\
\text { protégée. C. welensii est moins fréquent et particulièrement lié à des } \\
\text { gros chênes, notamment les vieux arbres des paysages agricoles } \\
\text { dans le Sud-Ouest. Comme C. cerdo, c'est une espèce ingénieur qui } \\
\text { facilite l'implantation d'autres espèces saproxyliques (Micó et al. 2015). } \\
\text { Espèce classée «quasi menacée» dans la Liste rouge européenne } \\
\text { (Cálix et al. 2018). }\end{array}$ & G & Fort \\
\hline & $\begin{array}{l}\text { Drymochares truquii } \\
\text { Mulsant, } 1847\end{array}$ & $\begin{array}{l}\text { Espèce aptère et endémique du Sud des Alpes (France et Italie). } \\
\text { Boisements de noisetiers et d'aulnes. Classée «en danger» dans la } \\
\text { Liste rouge européenne (Cálix et al. 2018). }\end{array}$ & G & Majeur \\
\hline & $\begin{array}{l}\text { Iberodorcadion arenarium } \\
\text { marsicanum Fracassi, } \\
1905\end{array}$ & Espèce aptère, localisée (en France) dans une vallée des Alpes du Nord. & G & Majeur \\
\hline & $\begin{array}{l}\text { Iberodorcadion fuliginator } \\
\quad \text { (Linnaeus, 1758) }\end{array}$ & $\begin{array}{l}\text { Longicorne aptère, de milieux herbacés, en régression dans toute la } \\
\text { France. La France constitue par ailleurs l'essentiel de son aire de } \\
\text { répartition (Berger 2012). Espèce déterminante ZNIEFF dans huit } \\
\text { régions (source INPN) et classé comme «en danger critique» dans la } \\
\text { Liste rouge du Limousin. }\end{array}$ & $\mathrm{E}$ & Fort \\
\hline
\end{tabular}




\begin{tabular}{|c|c|c|c|c|}
\hline \multicolumn{2}{|c|}{ Espèces à enjeux remarquables } & \multirow{2}{*}{$\begin{array}{l}\text { Commentaire } \\
\text { Longicorne aptère, méridional, de milieux herbacés, en regression. } \\
\text { La France constitue une part importante de son aire de répartition } \\
\text { (Berger 2012) }\end{array}$} & \multirow{2}{*}{$\frac{\text { EUNIS }}{E}$} & \multirow{2}{*}{$\begin{array}{l}\begin{array}{c}\text { Niveau } \\
\text { d'enjeu }\end{array} \\
\text { Fort }\end{array}$} \\
\hline & $\begin{array}{l}\text { Iberodorcadion molitor } \\
\text { (Fabricius, 1775) }\end{array}$ & & & \\
\hline & $\begin{array}{l}\text { Lamia textor } \\
\qquad(\text { Linnaeus, 1758) }\end{array}$ & $\begin{array}{l}\text { Longicorne largement répandu en Europe du Nord, mais localisé aux } \\
\text { zones humides et ripisylves (plante-hôte principale: saules), fraîches; } \\
\text { espèce peu mobile et en régression. Espèce déterminante ZNIEFF } \\
\text { dans six régions (source: INPN). }\end{array}$ & G & Fort \\
\hline & $\begin{array}{l}\text { Necydalis major } \\
\text { Linnaeus, } 1758\end{array}$ & $\begin{array}{l}\text { Espèce de zones fraiches, se développant dans les troncs d'arbres } \\
\text { feuillus. Très localisée en France, elle est cependant moins exigeante } \\
\text { que N. ulmi et moins rare à l'échelle de l'ensemble de l'Europe. Espèce } \\
\text { déterminante ZNIEFF dans trois régions (source: INPN) et classée } \\
\text { « quasi menacée» dans la Liste rouge européenne (Cálix et al. 2018). }\end{array}$ & G & Fort \\
\hline & $\begin{array}{l}\text { Necydalis ulmi } \\
\text { Chevrolat, } 1838\end{array}$ & $\begin{array}{l}\text { Espèce à large répartition méridionale, très fragmentée, typique des vieux } \\
\text { arbres feuillus à cavités, en forêt et en bocage, détectable par piégeage } \\
\text { aérien. Localisée et exigeante dans toute son aire de répartition. } \\
\text { Espèce déterminante ZNIEFF dans quatre régions (source: INPN) et } \\
\text { classée «vulnérable» dans la Liste rouge européenne des Coléoptères } \\
\text { saproxyliques (Cálix et al. 2018). }\end{array}$ & 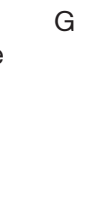 & Majeur \\
\hline & $\begin{array}{r}\text { Prinobius myardi } \\
\text { Mulsant, } 1842\end{array}$ & $\begin{array}{l}\text { Espèce méditerrannéenne, liées aux vieux arbres feuillus (souvent chêne } \\
\text { liège). En déclin comme ses arbres-hôtes. Espèce déterminante } \\
\text { ZNIEFF dans les deux régions où elle est présente, mais considérée } \\
\text { comme non menacée à l'échelle européenne (Cálix et al. 2018). }\end{array}$ & G & Fort \\
\hline & $\begin{array}{l}\text { Rhamnusium bicolor } \\
\quad \text { (Schrank, 1781) }\end{array}$ & $\begin{array}{l}\text { Espèce largement répartie, mais localisée et en déclin. Liée aux arbres } \\
\text { feuillus à cavités, souvent en parc ou alignement de bord de route. } \\
\text { Espèce déterminante ZNIEFF dans six régions (source: INPN) et } \\
\text { classée «vulnérable» dans la Liste rouge européenne des Coléoptères } \\
\text { saproxyliques (Cálix et al. 2018). }\end{array}$ & G & Fort \\
\hline & $\begin{array}{l}\text { Ropalopus ungaricus } \\
\quad \text { (Herst, 1784) }\end{array}$ & $\begin{array}{l}\text { Espèce révisée comprenant la ssp. gallicus des Alpes du Sud et la ssp. } \\
\text { ungaricus des Alpes du Nord, Jura et Vosges (Karpiński et al. 2020). } \\
\text { Liée aux vieux érables de montagne dépérissants. Les deux taxons } \\
\text { sont listées dans la Liste rouge européenne (Cálix et al. 2018): quasi } \\
\text { menacée et en danger. }\end{array}$ & G & Fort \\
\hline & $\begin{array}{l}\text { Stictoleptura erythroptera } \\
\text { (Hagenbach, 1822) }\end{array}$ & $\begin{array}{l}\text { Espèce à large répartition, très fragmentée, typique des vieux arbres à } \\
\text { cavités feuillus, en forêt et en bocage, détectable par piégeage aérien. } \\
\text { Localisée et exigeante dans toute son aire de répartition. Espèce } \\
\text { classée «vulnérable» dans la Liste rouge européenne des Coléoptères } \\
\text { saproxyliques (Cálix et al. 2018). }\end{array}$ & G & Majeur \\
\hline & $\begin{array}{l}\text { Tragosoma depsarium } \\
\text { (Linnaeus, 1767) }\end{array}$ & $\begin{array}{l}\text { Espèce relicte boréo-alpine, très localisée, dans les pinèdes à crochet et } \\
\text { pessières de haute montagne. Classée «quasi menacée » dans la Liste } \\
\text { rouge européenne (Cálix et al. 2018). }\end{array}$ & G & Majeur \\
\hline \multirow[t]{6}{*}{$\begin{array}{l}\text { Coléoptères } \\
\text { Scarabaeoidea }\end{array}$} & $\begin{array}{l}\text { Aesalus scarabaeoides } \\
\text { a (Panzer, 1795) }\end{array}$ & $\begin{array}{l}\text { Petite espèce de lucane des vieilles forêts, liée au vieux bois mort } \\
\text { décomposé de feuillus. Large répartition mais très localisée. Cette espèce } \\
\text { est un indicateur de naturalité des forêts. Déterminante ZNIEFF dans six } \\
\text { régions (source: INPN). Classée comme «quasi menacée» dans la Liste } \\
\text { rouge européenne des Coléoptères saproxyliques (Cálix et al. 2018). }\end{array}$ & $\mathrm{G}$ & Fort \\
\hline & $\begin{array}{l}\text { Aphodius coniugatus } \\
\quad \text { (Panzer, 1795) }\end{array}$ & Bousier endocopride, localisé, peu commun et très reconnaissable. & $\mathrm{E}$ & Fort \\
\hline & $\begin{array}{l}\text { Calicnemis latreillii } \\
\text { Laporte de Castelnau, } \\
1832\end{array}$ & $\begin{array}{l}\text { Espèce se développant dans les bois flottés du littoral méditerranéen. } \\
\text { Très sensible au nettoyage des plages. Localisée et en régression. } \\
\text { Espèce déterminante ZNIEFF dans l'ex-région Languedoc-Roussillon. }\end{array}$ & $\mathrm{B}$ & Majeur \\
\hline & $\begin{array}{l}\text { Calicnemis obesa } \\
\quad(\text { Erichson, 1841) }\end{array}$ & $\begin{array}{l}\text { Espèce se développant dans les bois flottés du littoral atlantique. Très } \\
\text { sensible au nettoyage des plages. Localisée et en régression. Espèce } \\
\text { déterminante ZNIEFF en Nouvelle-Aquitaine. Présente aussi en Vendée. }\end{array}$ & $\mathrm{B}$ & Majeur \\
\hline & $\begin{array}{l}\text { Ceruchus chrysomelinus } \\
\text { (Hochenwarth, 1785) }\end{array}$ & $\begin{array}{l}\text { Espèce des vieilles forêts, sub-montagnardes à montagnardes, entre } \\
300 \text { et } 1500 \text { mètres d'altitude, relictuelle et rare. Elle constitue un } \\
\text { indicateur de naturalité des forêts de montagne. Classée comme } \\
\text { «quasi menacée» dans la Liste rouge européenne des Coléoptères } \\
\text { saproxyliques (Cálix et al. 2018). }\end{array}$ & $\mathrm{G}$ & Majeur \\
\hline & $\begin{array}{l}\text { Cetonischema } \\
\text { speciosissima } \\
\text { (Scopoli, 1786) }\end{array}$ & $\begin{array}{l}\text { Espèce à large répartition mais localisée et généralement peu abondante. } \\
\text { En France, elle est liée aux cavités d'arbres feuillus, essentiellement en } \\
\text { contexte forestier. Déterminante ZNIEFF dans six régions françaises } \\
\text { (source: INPN). Classée « quasi menacée» dans la Liste rouge } \\
\text { européenne des Coléoptères saproxyliques (Cálix et al. 2018). }\end{array}$ & $\mathrm{G}$ & Fort \\
\hline
\end{tabular}




\begin{tabular}{|c|c|c|c|c|}
\hline \multirow{2}{*}{\multicolumn{2}{|c|}{$\begin{array}{r}\text { Espèces à enjeux remarquables } \\
\text { Eupotosia mirifica } \\
\text { (Mulsant, 1842) }\end{array}$}} & \multirow[b]{2}{*}{$\begin{array}{l}\text { Commentaire } \\
\text { Cétoine du pourtour méditerranén, très localisée, liée aux arbres à cavité, } \\
\text { notamment en chênaie pubescente (et aux accumulations de matière } \\
\text { organique dans les karsts du bois de Païolive, Ardèche, principale } \\
\text { localité française). Très reconnaissable et à enjeux de conservation déjà } \\
\text { reconnus (Tassi et al. 2004). Classée « vulnérable» dans la Liste rouge } \\
\text { européenne des Coléoptères saproxyliques (Cálix et al. 2018). }\end{array}$} & \multirow[b]{2}{*}{ EUNIS } & \multirow{2}{*}{$\begin{array}{l}\begin{array}{c}\text { Niveau } \\
\text { d'enjeu }\end{array} \\
\text { Majeur }\end{array}$} \\
\hline & & & & \\
\hline & $\begin{array}{l}\text { Gnorimus variabilis } \\
\text { (Linnaeus, 1758) }\end{array}$ & $\begin{array}{l}\text { Cétoine des arbres à cavité, en forêts et bocages. Largement répandue } \\
\text { mais localisée (Tauzin 2004). En déclin comme les biotopes } \\
\text { susceptibles de l'abriter. Déterminante ZNIEFF dans cinq régions } \\
\text { (source: INPN). Classée comme « quasi menacée» dans la Liste rouge } \\
\text { européenne des Coléoptères saproxyliques (Cálix et al. 2018). }\end{array}$ & $\mathrm{G}$ & Fort \\
\hline & $\begin{array}{l}\text { Gymnopleurus sturmii } \\
\text { (MacLeay, 1821) }\end{array}$ & $\begin{array}{l}\text { Bousier rouleur, en déclin, même en Corse (Bordat et al. 2020). Classée } \\
\text { comme «quasi menacée» dans la Liste rouge rouge méditerranéenne } \\
\text { (Numa et al. 2020). }\end{array}$ & $E, F$ & Fort \\
\hline & $\begin{array}{l}\text { Liocola marmorata } \\
\text { (Fabricius, 1792) }\end{array}$ & $\begin{array}{l}\text { Espèce à large répartition, plutôt septentrionale, liée aux cavités d'arbres } \\
\text { feuillus dans les futaies et les vieux parcs; localisée et peu abondante. } \\
\text { Espèce déterminante ZNIEFF dans quatre régions (source: INPN) mais } \\
\text { non menacée à l'échelle européenne (Cálix et al. 2018). }\end{array}$ & $\mathrm{G}$ & Fort \\
\hline & $\begin{array}{l}\text { Scarabaeus sacer } \\
\text { Linnaeus, } 1758\end{array}$ & $\begin{array}{l}\text { Bousier rouleur, en déclin fort sur le continent; plus courant mais localisé } \\
\text { aux zones littorales en Corse (Lumaret 1990). Espèce déterminante } \\
\text { ZNIEFF dans les deux régions où elle est présente (source: INPN). Non } \\
\text { évaluée dans la Liste rouge méditerranéenne (Numa et al. 2020). }\end{array}$ & $B, E$ & Majeur \\
\hline \multicolumn{3}{|c|}{$\begin{array}{l}\text { Scarabaeus semipunctatus Bousier rouleur des zones dunaires, localisé et en régression. Espèce } \\
\begin{array}{l}\text { Fabricius, } 1792 \\
\text { classée «vulnérable» dans la Liste rouge méditerranéenne (Numa et al. } \\
\text { 2020). }\end{array}\end{array}$} & $B$ & Majeur \\
\hline & $\begin{array}{l}\text { Thorectes albarracinus } \\
\text { Wagner, } 1928\end{array}$ & $\begin{array}{l}\text { Endémique de l'Est des Pyrénées. Espèce aptère et localisée. Espèce } \\
\text { listée comme «quasi menacée » dans la Liste rouge méditerranéenne } \\
\text { (Numa et al. 2020). }\end{array}$ & $\mathrm{E}(\mathrm{F}, \mathrm{G})$ & Fort \\
\hline & $\begin{array}{l}\text { Thorectes sardous } \\
\text { Erichson, } 1845\end{array}$ & $\begin{array}{l}\text { Endémique corso-sarde. Aptère, très localisée en quelques populations } \\
\text { sur le littoral de Corse. Espèce classée comme «en danger » dans la } \\
\text { Liste rouge méditerranéenne (Numa et al. 2020). }\end{array}$ & B & Majeur \\
\hline & $\begin{array}{l}\text { Thorectes sericeus } \\
\text { (Jeckel, 1865) }\end{array}$ & $\begin{array}{l}\text { Bousier aptère, endémique du littoral landais, localisé sur les habitats de } \\
\text { dune grise. }\end{array}$ & B & Majeur \\
\hline \multirow[t]{5}{*}{$\begin{array}{l}\text { Coléoptères } \\
\text { Tenebrionidae }\end{array}$} & $\begin{array}{l}\text { Akis bacarozzo } \\
\quad(\text { Schrank, 1786) }\end{array}$ & $\begin{array}{l}\text { Seulement 20-25 localités en France continentale, la plupart étant des } \\
\text { citations anciennes (<1980) (Soldati \& Soldati sous presse). En nette } \\
\text { régression par destruction de ses habitats. Encore abondante et non } \\
\text { menacée en Corse. Identification facile à vue. }\end{array}$ & $\mathrm{H}, \mathrm{J}$ & Fort \\
\hline & $\begin{array}{l}\text { Blaps gigas } \\
\quad \text { (Linnaeus, 1767) }\end{array}$ & $\begin{array}{l}\text { Espèce synanthrope méditerranéenne en nette régression de par la } \\
\text { destruction de ses habitats (Soldati 2007), beaucoup d'observations } \\
\text { anciennes mais non revue depuis des décennies dans plusieurs } \\
\text { départements: Alpes-Maritimes, Ardèche, Gard, Var et Vaucluse. } \\
\text { En Corse, signalée d'une dizaine de localités, incluant les données } \\
\text { historiques, mais uniquement observée récemment à Bonifacio } \\
\text { (Soldati \& Soldati sous presse). Identification facile à vue ou sur photo } \\
\text { envoyée à un expert. }\end{array}$ & $\mathrm{B}, \mathrm{H}, \mathrm{J}$ & Fort \\
\hline & $\begin{array}{l}\text { Gonocephalum calcaripes } \\
\text { (Karsch, 1881) }\end{array}$ & $\begin{array}{l}\text { Espèce seulement présente dans huit localités de l'Aude, du Var et de } \\
\text { l'Hérault, essentiellement littorales (Soldati \& Soldati sous presse), } \\
\text { une localité de l'Aude récemment détruite par des aménagements } \\
\text { (zone industrialo-portuaire). Plusieurs stations de quelques centaines } \\
\text { de m² situées à côté de zones en construction. Identification difficile } \\
\text { (genitalia); mais Faune de France à paraître. }\end{array}$ & $B$ & Majeur \\
\hline & $\begin{array}{l}\text { Gonocephalum pygmaeum } \\
\text { (Steven, 1829) }\end{array}$ & $\begin{array}{l}\text { Espèce ripicole, des bancs limoneux des cours d'eau du Bassin du } \\
\text { Rhône et en région méditerranéenne, en très nette régression du fait } \\
\text { des pollutions d'origine agricole ou de l'aménagement des rives et } \\
\text { des berges; n'a pas été revue depuis plus de } 50 \text { ans dans la plupart } \\
\text { de ses départements d'occurrence. Identification difficile, sous } \\
\text { stéréomicroscope, mais envisageable sur photo par un expert. }\end{array}$ & C & Majeur \\
\hline & $\begin{array}{l}\text { Heliopates littoralis } \\
\text { Español, } 1958\end{array}$ & $\begin{array}{l}\text { Espèce des dunes littorales présente dans seulement quelques } \\
\text { localités des Pyrénées-Orientales et de l'Aude, dans des zones } \\
\text { très touristiques et très fréquentées, avec des menaces réelles } \\
\text { (destruction des habitats, constructions à proximité) (Soldati \& Soldati } \\
\text { sous presse). Identification assez facile sous stéréomicroscope ou sur } \\
\text { photo. }\end{array}$ & $B$ & Majeur \\
\hline
\end{tabular}


ANNEXE 2. - Suite.

\begin{tabular}{|c|c|c|c|c|}
\hline \multirow{2}{*}{\multicolumn{2}{|c|}{$\begin{array}{r}\text { Espèces à enjeux remarquables } \\
\begin{array}{r}\text { Leptoderis collaris } \\
(\text { Linnaeus, } 1767)\end{array}\end{array}$}} & \multirow{2}{*}{$\begin{array}{l}\text { Commentaire } \\
\text { Troglophile, en nette régression: destruction et anthropisation de ses } \\
\text { habitats. Non revue dans les Pyrénées-Orientales depuis } 1958 \text { (Soldati } \\
\text { 2007). Retrouvé dans le Var (Ponel et al. 2020) après plus d'un siècle } \\
\text { sans observation. Identification facile. }\end{array}$} & \multirow{2}{*}{$\begin{array}{c}\text { EUNIS } \\
\text { B, H, J }\end{array}$} & \multirow{2}{*}{$\begin{array}{c}\begin{array}{c}\text { Niveau } \\
\text { d'enjeu }\end{array} \\
\text { Majeur }\end{array}$} \\
\hline & & & & \\
\hline & $\begin{array}{l}\text { Phaleria acuminata } \\
\text { Küster, } 1852\end{array}$ & $\begin{array}{l}\text { Psammohalophile des plages et des dunes littorales, connue en France } \\
\text { uniquement de cinq localités isolées en Corse et d'une localité du Var } \\
\text { (Hyères), observations historiques inclues (Soldati } 2007 \text {; Soldati \& } \\
\text { Soldati sous presse). Certaines stations < quelques km², pouvant être } \\
\text { facilement impactées. Identification difficile. }\end{array}$ & B & Majeur \\
\hline & $\begin{array}{c}\text { Phaleria atlantica } \\
\text { Fauvel, } 1899\end{array}$ & $\begin{array}{l}\text { Espèce des plages et des dunes du littoral extrême sud atlantique, } \\
\text { connue seulement de huit stations des Landes et des Pyrénées- } \\
\text { Atlantiques, incluant les observations historiques; certaines localités } \\
\text { détruites (Thomas 2014). Devenue sporadique et trés rare, en voie de } \\
\text { disparition, avec comme principales menaces avérées: destruction de } \\
\text { l'habitat, piétinement par surfréquentation, raclage des laisses de mer. } \\
\text { Identification difficile mais Faune de France à paraître (Soldati \& Soldati } \\
\text { sous presse). }\end{array}$ & B & Fort \\
\hline & $\begin{array}{l}\text { Phaleria insulana } \\
\text { Rey, } 1890\end{array}$ & $\begin{array}{l}\text { Espèce psammohalophile vivant sous les laisses des plages, endémique } \\
\text { corso-sarde, connue d'une dizaine de localités isolées en Corse (Soldati } \\
\text { 2007; Soldati \& Soldati sous presse). Menacée par la surfréquentation } \\
\text { touristique, le ramassage des laisses de mer. Identification difficile mais } \\
\text { Faune de France à paraître (Soldati \& Soldati sous presse). }\end{array}$ & B & Fort \\
\hline & $\begin{array}{l}\text { Pedinus femoralis } \\
\quad \text { (Linnaeus, 1767) }\end{array}$ & $\begin{array}{l}\text { Sur les } 11 \text { stations historiques connues en France, seulement observée } \\
\text { dans une seule en } 2000 \text {, dans une zone sableuse en bordure de cours } \\
\text { d'eau (Soldati \& Soldati sous presse). Espèce sensible paraissant } \\
\text { en nette voie de disparition en France. Identification difficile, sous } \\
\text { stéréomicroscope, mais envisageable sur photo par un expert. }\end{array}$ & $C, E$ & Fort \\
\hline & $\begin{array}{l}\text { Pimelia muricata cyrnensis } \\
\text { F. Soldati \& Rougon, } \\
2017\end{array}$ & $\begin{array}{l}\text { Endémique, initialement décrite de la côte orientale de la Corse (Soldati \& } \\
\text { Rougon 2017), mais aussi présente sur le littoral sableux du Var. Très } \\
\text { peu de localités de présence. Stations souvent très réduites (quelques } \\
\text { centaines de } \mathrm{m}^{2} \text { à quelques } \mathrm{km}^{2} \text { ) et très fragiles face à la pression } \\
\text { anthropique. Paraît avoir disparu des Alpes-Maritimes. Identification } \\
\text { envisageable à vue ou sur photo par un expert. }\end{array}$ & B & Majeur \\
\hline & $\begin{array}{l}\text { Pseudoseriscius pruinosus } \\
\text { (Dufour, 1820) }\end{array}$ & $\begin{array}{l}\text { Espèce psammohalophile des dunes littorales, connue en France } \\
\text { uniquement du lido entre Sète et Agde (Soldati } 2007 \text {; Soldati \& Soldati } \\
\text { sous presse). Zone réduite à quelques } \mathrm{km}^{2} \text {, soumise à forte pression: } \\
\text { fréquentation touristique élevée, routes et constructions en arrière } \\
\text { empêchant le recul naturel du trait de côte. Identification assez facile, } \\
\text { sous stéréomicroscope. }\end{array}$ & B & Majeur \\
\hline & $\begin{array}{l}\text { Scaurus tristis } \\
\text { A. G. Olivier, } 1795\end{array}$ & $\begin{array}{l}\text { < } 30 \text { localités au niveau national, toutes en zone thermo-méditerranéenne, } \\
\text { la plupart historiques (Soldati \& Soldati sous presse). Troglophile et } \\
\text { souvent synanthrope, menacée par la destruction et l'aménagement de } \\
\text { ses habitats. Identification envisageable à vue. }\end{array}$ & $\mathrm{B}, \mathrm{H}, \mathrm{J}$ & Majeur \\
\hline & $\begin{array}{l}\text { Tentyria ligurica Solier, } \\
1835\end{array}$ & $\begin{array}{l}\text { Espèce des dunes littorales. Seulement cinq localités dans le golfe } \\
\text { d'Ajaccio (Soldati } 2007 \text {; Soldati \& Soldati sous presse). Forte pression } \\
\text { touristique, certaines stations étant réduites à quelques centaines } \\
\text { de } \mathrm{m}^{2} \text {. Autres menaces: destruction des habitats, constructions à } \\
\text { proximité. Identification difficile mais Faune de France à paraître. }\end{array}$ & B & Fort \\
\hline & $\begin{array}{l}\text { Xanthomus pallidus } \\
\text { (Curtis, 1830) }\end{array}$ & $\begin{array}{l}\text { Espèce psammohalophile encore bien présente dans les dunes littorales } \\
\text { de l'Atlantique et de la Manche. En revanche, elle ne paraît encore } \\
\text { présente que dans trois localités isolées du littoral méditerranéen et } \\
\text { dans trois localités corses (Soldati 2007; Soldati \& Soldati sous presse); } \\
\text { ces dernières se trouvant essentiellement dans des zones touristiques } \\
\text { très fréquentées (donc menacées). Identification assez facile sous } \\
\text { stéréomicroscope, envisageable aussi sur photo par un expert. }\end{array}$ & B & Fort \\
\hline $\begin{array}{l}\text { Hétéroptères } \\
\text { Pentatomoidea }\end{array}$ & $\begin{array}{l}\text { Aelia klugii Hahn, } 1833 \\
\text { a }\end{array}$ & $\begin{array}{l}\text { Espèce à distribution très morcelée, notamment en plaine où plusieurs } \\
\text { de ses stations historiques ont disparues. Elle vit dans les marais, les } \\
\text { tourbières et les pelouses d'altitude, toujours dans des ensembles } \\
\text { d'habitats préservés. }\end{array}$ & $D, E$ & Fort \\
\hline & $\begin{array}{l}\text { Antheminia lunulata } \\
(\text { Goeze, 1778) }\end{array}$ & $\begin{array}{l}\text { Distribution très morcelée en France (<25 stations), avec plusieurs } \\
\text { disparitions régionales avérées, notamment en plaine. Cette espèce } \\
\text { habite les pelouses sub-steppiques sous influence continentale. Elle } \\
\text { est également considérée comme menacée d'extinction en Allemagne } \\
\text { (Esser 2011). }\end{array}$ & $E$ & Majeur \\
\hline
\end{tabular}




\begin{tabular}{|c|c|c|c|}
\hline Espèces à enjeux remarquables & Commentaire & EUNIS & $\begin{array}{l}\text { Niveau } \\
\text { d'enjeu }\end{array}$ \\
\hline $\begin{array}{l}\text { Brachynema cinctum } \\
\text { (Fabricius, 1775) }\end{array}$ & $\begin{array}{l}\text { Espèce méditerranéenne, colonisant les sansouires et marais côtiers. } \\
\text { Facile d'identification, cette espèce localement abondante a déjà } \\
\text { disparue de plusieurs stations littorales. D'autres localités sont } \\
\text { soumises à de fortes pressions d'usage. }\end{array}$ & $A, B$ & Fort \\
\hline $\begin{array}{l}\text { Cephalocteus } \\
\text { scarabaeoides } \\
\text { (Fabricius, 1803) }\end{array}$ & $\begin{array}{l}\text { Espèce sud et ouest-méditerranéenne, toujours localisée et morcelée } \\
\text { dans son aire. La seule population connue en France se situait dans } \\
\text { les dunes des environs de Marseille (Amyot \& Audinet-Serville 1843), } \\
\text { d'où elle semble avoir disparue (Lupoli \& Dusoulier 2015). En plus de ce } \\
\text { déclin, cette espèce sténotope vit uniquement dans les dunes blanches } \\
\text { sous climat thermoméditerranéen. }\end{array}$ & 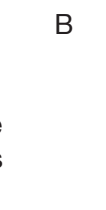 & Majeur \\
\hline $\begin{array}{l}\text { Elasmucha fieberi } \\
\text { (Jakovlev, 1865) }\end{array}$ & $\begin{array}{l}\text { Distribution eurosibérienne très fragmentée à l'ouest et au sud de son } \\
\text { aire. En France < } 30 \text { localités dans des bétulaies préservées dans le } \\
\text { Nord, ou en altitude plus au sud (Lupoli \& Dusoulier 2015). Son aire } \\
\text { morcelée et son association avec des boisements frais et préservés en } \\
\text { font une espèce sensible et menacée. }\end{array}$ & G & Fort \\
\hline $\begin{array}{l}\text { Eurydema herbacea } \\
\quad \text { (Herrich-Schäffer, 1834) }\end{array}$ & $\begin{array}{l}\text { Taxon à distribution atlantique, strictement localisé aux plages sableuses } \\
\text { littorales sur lesquelles il se nourrit sur Cakile, depuis la zone des } \\
\text { laisses de mer jusqu'aux dunes mobiles. N'a pas été revu dans } \\
\text { plusieurs de ses stations historiques et potentiellement menacé par la } \\
\text { fréquentation et les aménagements. }\end{array}$ & $B$ & Fort \\
\hline $\begin{array}{l}\text { Eurydema sea Baena, } \\
\text { Péricart \& De la Rosa, } \\
2004\end{array}$ & $\begin{array}{l}\text { Espèce subendémique des reliefs calcaires méditerranéens du Nord-Est } \\
\text { de l'Espagne, seulement connue d'une seule station des Pyrénées- } \\
\text { Orientales (Sannier 2019). Sténotope des rocailles, crêtes et falaises } \\
\text { sur lesquelles elle se nourrit strictement de la Crucifère épineuse } \\
\text { Hormathophylla spinosa (L.) P.Küpfer. }\end{array}$ & $\mathrm{H}$ & Majeur \\
\hline $\begin{array}{l}\text { Eurygaster hottentotta } \\
\quad \text { (Fabricius, 1775) }\end{array}$ & $\begin{array}{c}<30 \text { stations en France, toutes dans des pelouses, garrigues et friches } \\
\text { thermoxériques du secteur méditerranéen. Les populations, toujours } \\
\text { faibles, sont fortement menacées par la destruction de ses habitats. }\end{array}$ & $E$ & Fort \\
\hline $\begin{array}{l}\text { Leprosoma inconspicuum } \\
\text { Baerensprung, } 1859\end{array}$ & $\begin{array}{l}\text { Aire très morcelée et }<25 \text { stations en France, dans des habitats sub- } \\
\text { steppiques herbacés et rocailleux de l'étage supraméditerranéen. Très } \\
\text { sténotope, ce taxon est menacé par les installations photovoltaïques et } \\
\text { éoliennes. }\end{array}$ & $\mathrm{H}, \mathrm{E}$ & Fort \\
\hline $\begin{array}{l}\text { Phimodera humeralis } \\
\text { (Dalman, 1823) }\end{array}$ & $\begin{array}{l}\text { Espèce toujours très localisée ou en situation de déclin dans toute son } \\
\text { aire. Elle figure sur plusieurs listes rouges comme «en danger critique } \\
\text { d'extinction» en Allemagne (Günther et al. 1998), ou «vulnérable» au } \\
\text { Danemark (Tolsgaard 1998) et en Finlande (Rintala \& Rinne 2011). Elle } \\
\text { est considérée disparue en Autriche (Rabitsch 2002) et en République } \\
\text { tchèque (Kment \& Vilímová 2005). En France et aux Pays-Bas, elle a été } \\
\text { redécouverte ces dernières années (Aukema 2010; Lessieur \& Lupoli } \\
\text { 2018; Dusoulier et al. sous presse) mais demeure très menacée. En France } \\
\text { où son aire se restreint au littoral aquitain, elle est sténotope, colonisant } \\
\text { seulement les zones de sables libres des dunes blanches et les marges de } \\
\text { dune grise. Plusieurs stations historiques semblent avoir disparues. }\end{array}$ & 20 & Majeur \\
\hline $\begin{array}{l}\text { Phimodera flori } \\
\text { Fieber, } 1863\end{array}$ & $\begin{array}{l}\text { Espèce à distribution eurosibérienne se comportant en boréo-alpine } \\
\text { à l'ouest de son aire. En France, extrêmement localisée avec deux } \\
\text { secteurs de présence: massifs internes des Alpes méridionales (Queyras, } \\
\text { Briançonnais) et plateaux steppiques d'altitude de Cerdagne. Elle vit } \\
\text { dans des habitats dominés par des affleurements rocheux ou des } \\
\text { pelouses rases avec un recouvrement végétal au sol épars (Lupoli \& } \\
\text { Dusoulier 2015). Stations xérothermophiles bénéficiant d'hiver froid. }\end{array}$ & $\mathrm{H}, \mathrm{E}$ & Majeur \\
\hline $\begin{array}{l}\text { Rhacognathus punctatus } \\
\quad \text { (Linnaeus, 1758) }\end{array}$ & $\begin{array}{l}\text { Vaste aire mais distribution très morcelée en France. Ce taxon vit } \\
\text { uniquement dans des habitats préservés et de qualité continue depuis } \\
\text { longtemps (Ramsay 2013): vieilles landes à bruyères, marais, lisières } \\
\text { forestières. Espèce prédatrice. }\end{array}$ & $\mathrm{F}$ & Fort \\
\hline $\begin{array}{l}\text { Sciocoris umbrinus } \\
\text { (Wolff, 1804) }\end{array}$ & $\begin{array}{l}\text { Populations très morcelées, rarissime en France ( }<20 \text { stations). } \\
\text { Sténotope dans des milieux frais et humides bien conservés: bordure } \\
\text { de marais, tourbières et pelouses d'altitude. Plusieurs stations } \\
\text { disparues, notamment en plaine. }\end{array}$ & $\mathrm{D}, \mathrm{E}$ & Fort \\
\hline $\begin{array}{l}\text { Sternodontus obtusus } \\
\text { Mulsant \& Rey, } 1856\end{array}$ & $\begin{array}{l}\text { Espèce à distribution ponto-européenne mais aux populations très } \\
\text { morcelées. En France, l'espèce est répandue dans les Alpes du Sud, } \\
\text { la Provence et dans les Causses. Écologie stricte: rocailles, coteaux et } \\
\text { falaises à forte minéralité. Se nourrit sur Laserpitium gallicum L. dans } \\
\text { les zones instables et d'éboulis. En régression dans les secteurs les } \\
\text { plus chauds de l'aire. }\end{array}$ & $\mathrm{H}$ & Fort \\
\hline
\end{tabular}


ANNEXE 2. - Suite.

\begin{tabular}{|c|c|c|c|c|}
\hline \multicolumn{2}{|c|}{ Espèces à enjeux remarquables } & \multirow[b]{2}{*}{$\begin{array}{l}\text { Commentaire } \\
\text { Distribution strictement ouest-méditerranéenne. En France, elle était } \\
\text { répandue dans toute l'aire méditerranéenne au 19e siècle et début du } \\
20 \text { e siècle (Lupoli \& Dusoulier 2015). Une seule observation récente } \\
\text { (2000) dans la région du Salagou, ce qui démontre une très forte } \\
\text { régression d'autant que l'espèce est facile à détecter et à identifier. Elle } \\
\text { colonise des garrigues et pelouses. La disparition de près de } 100 \% \\
\text { des stations historiques reste difficile à expliquer. }\end{array}$} & \multirow{2}{*}{$\frac{\text { EUNIS }}{E, F}$} & \multirow{2}{*}{$\begin{array}{l}\begin{array}{c}\text { Niveau } \\
\text { d'enjeu }\end{array} \\
\text { Majeur }\end{array}$} \\
\hline & $\begin{array}{l}\text { Ventocoris falcatus } \\
\text { (Cyrillus, 1787) }\end{array}$ & & & \\
\hline \multirow[t]{10}{*}{$\begin{array}{l}\text { Lépidoptères } \\
\text { diurnes } \\
\text { (Rhopalocères } \\
\text { et Zygaena) }\end{array}$} & $\begin{array}{l}\text { Boloria selene (Denis \& } \\
\text { Schiffermüller, 1775) }\end{array}$ & $\begin{array}{l}\text { Distribution holarctique; la régression de cette espèce en France est } \\
\text { nette: « disparue» en Haute-Normandie, «en danger critique» en } \\
\text { Picardie, «en danger» en Bretagne et en Île-de-France (ainsi que sur } \\
\text { la future Liste rouge des Pays de la Loire), «vulnérable» en Poitou- } \\
\text { Charentes et «quasi menacée» dans cinq autres régions. }\end{array}$ & $\mathrm{D}, \mathrm{E}, \mathrm{F}$ & Fort \\
\hline & $\begin{array}{l}\text { Carcharodus baeticus } \\
\text { (Rambur, [1839]) }\end{array}$ & $\begin{array}{l}\text { Distribution ouest-méditerranéenne; espèce localisée, liée aux milieux } \\
\text { xériques, voire steppiques. Sa conservation passe par la sauvegarde } \\
\text { du pastoralisme extensif, qui maintient une végétation herbacée rase } \\
\text { et favorise la plante-hôte larvaire, le Marrube vulgaire Marrubium } \\
\text { vulgare L. Vulnérable sur la Liste rouge nationale. }\end{array}$ & E & Fort \\
\hline & $\begin{array}{l}\text { Chazara briseis } \\
\quad \text { (Linnaeus, 1764) }\end{array}$ & $\begin{array}{l}\text { Distribution méditerranéo-asiatique; espèce affectionnant les pelouses } \\
\text { xérophiles rases et rocailleuses, de préférence calcaires, avec un } \\
\text { pâturage modéré. La plupart de ses populations situées en plaine } \\
\text { se sont effondrées en seconde moitié du vingtième siècle. La } \\
\text { régression atteint désormais ses bastions du Sud. Vulnérable en } \\
\text { France. }\end{array}$ & E & Fort \\
\hline & $\begin{array}{l}\text { Coenonympha hero } \\
\text { (Linnaeus, 1761) }\end{array}$ & $\begin{array}{l}\text { Distribution eurasiatique; décrite comme banale en Île-de-France au } \\
\text { xIxe siècle, cette espèce ne subsiste plus qu'en Franche-Comté, où ses } \\
\text { habitats sont menacés par l'enrésinement, le drainage ou les coupes } \\
\text { franches en forêt. En danger critique d'extinction en France. }\end{array}$ & $D, E$ & Majeur \\
\hline & $\begin{array}{l}\text { Coenonympha oedippus } \\
\text { (Fabricius, 1787) }\end{array}$ & $\begin{array}{l}\text { Distribution eurasiatique, avec une aire de répartition fragmentée dans } \\
\text { l'Ouest de l'Europe; hormis dans le massif forestier des Landes de } \\
\text { Gascogne, espèce très menacée partout, en particulier par le drainage } \\
\text { des prairies marécageuses. Disparue en Occitanie, Île-de-France et } \\
\text { Pays de la Loire. }\end{array}$ & $D, E$ & Fort \\
\hline & $\begin{array}{l}\text { Coenonympha tullia } \\
\text { (Müller, 1764) }\end{array}$ & $\begin{array}{l}\text { Distribution eurasiatique; cette espèce relicte post-glaciaire vit } \\
\text { exclusivement dans les tourbières à linaigrettes, le plus souvent } \\
\text { au-dessus de } 800 \text { m d'altitude. Non revue depuis } 2003 \text { dans le Morvan, } \\
\text { elle est au bord de l'extinction partout, y compris dans ses bastions } \\
\text { francs-comtois ou vosgiens, menacée par l'enrésinement et les } \\
\text { drainages. }\end{array}$ & $\mathrm{D}, \mathrm{E}$ & Majeur \\
\hline & $\begin{array}{l}\text { Euphydryas maturna } \\
\text { (Linnaeus, 1758) }\end{array}$ & $\begin{array}{l}\text { Distribution eurosibérienne; encore bien présente en région parisienne } \\
\text { dans les années 1960, l'espèce est aujourd'hui essentiellement } \\
\text { cantonnée en Bourgogne. En danger sur la Liste rouge nationale. } \\
\text { En danger critique en région Centre-Val-de-Loire, elle est menacée } \\
\text { partout par certaines pratiques agricoles et sylvicoles, en particulier la } \\
\text { monoculture de résineux. En plaine, elle subit une extinction de grande } \\
\text { envergure. }\end{array}$ & $\mathrm{G}, \mathrm{F}$ & Majeur \\
\hline & $\begin{array}{l}\text { Euphydryas desfontainii } \\
\text { (Godart, 1819) }\end{array}$ & $\begin{array}{l}\text { Distribution ouest-méditerranéenne, en limite de répartition dans l'Est des } \\
\text { Pyrénées françaises; espèce présente sur des anciennes terrasses, qui } \\
\text { peuvent être menacées par l'enfrichement, mais elle semble conquérir } \\
\text { quelques biotopes, peut-être favorisée par le changement climatique } \\
\text { en cours. "Vulnérable» en Liste rouge nationale. }\end{array}$ & E & Fort \\
\hline & $\begin{array}{l}\text { Gegenes pumilio } \\
\quad \text { (Hoffmannsegg, 1804) }\end{array}$ & $\begin{array}{l}\text { Distribution arabo-méditerranéenne et africaine; en France, } \\
\text { historiquement limité entre Nice et Toulon à la série de l'oléolentisque, } \\
\text { habitat côtier à Euphorbes arborescentes Euphorbia dendroides. } \\
\text { Ces stations sont toutes menacées par une urbanisation intense et } \\
\text { le papillon n'a pas été revu en France depuis les années 1990. La } \\
\text { découverte d'une population passée inaperçue présenterait un enjeu } \\
\text { majeur. }\end{array}$ & $F, E$ & Majeur \\
\hline & $\begin{array}{l}\text { Hyponephele lupina } \\
\text { (O. Costa, 1836) }\end{array}$ & $\begin{array}{l}\text { Espèce méditerranéenne sarmatique présente dans deux régions, où elle } \\
\text { est jugée «en danger»; ses populations sont localisées à des habitats } \\
\text { steppiques, soumis à de fortes pressions immobilières, implantation } \\
\text { de zones d'activités ou d'infrastructures routières, voire de centrales } \\
\text { photovoltaïques de grande ampleur. }\end{array}$ & E & Fort \\
\hline
\end{tabular}




\begin{tabular}{|c|c|c|c|}
\hline Espèces à enjeux remarquables & Commentaire & EUNIS & $\begin{array}{l}\text { Niveau } \\
\text { d'enjeu }\end{array}$ \\
\hline $\begin{array}{l}\text { Iberochloe tagis } \\
\text { (Hübner, [1804]) }\end{array}$ & $\begin{array}{l}\text { Distribution atlanto-méditerranéenne; Piéride printanière peu commune, } \\
\text { inféodée à des habitats xériques, tels que pelouses caillouteuses } \\
\text { ou garrigues, où prospèrent ses plantes-hôtes larvaires, les lberis. } \\
\text { Menacée par la fermeture des milieux, l'intensification de l'agriculture } \\
\text { - en particulier viticole - ou la multiplication des centrales photo- } \\
\text { voltaïques en zone naturelle. }\end{array}$ & $\mathrm{E}, \mathrm{H}$ & Fort \\
\hline $\begin{array}{l}\text { Lopinga achine } \\
\text { (Scopoli, 1763) }\end{array}$ & $\begin{array}{l}\text { Distribution eurasiatique; cette espèce est présente exclusivement dans } \\
\text { des vieilles forêts de feuillus et sa survie nécessite une gestion en } \\
\text { taillis sous futaie ainsi que le maintien d'une strate herbacée. Classée } \\
\text { « disparue» dans cinq régions (ainsi que sur la future Liste rouge des } \\
\text { Pays de la Loire). }\end{array}$ & $\mathrm{G}$ & Fort \\
\hline $\begin{array}{l}\text { Phengaris teleius } \\
\quad \text { (Bergsträsser, [1779]) }\end{array}$ & $\begin{array}{l}\text { Distribution eurasiatique; ce lycène myrmécophile obligatoire est } \\
\text { menacé par la régression des zones humides qui abritent sa plante- } \\
\text { hôte larvaire la Sanguisorbe officinale Sanguisorba officinalis L., } \\
\text { mais également par leur fermeture, préjudiciable au maintien des } \\
\text { fourmilières parasitées. }\end{array}$ & $E, D$ & Majeur \\
\hline $\begin{array}{l}\text { Tomares ballus } \\
\text { (Fabricius, 1787) }\end{array}$ & $\begin{array}{l}\text { Distribution ouest-méditerranéenne; espèce localisée à des biotopes } \\
\text { alluvionnaires ou sédimentaires tous situés entre l'Est des Bouches-du- } \\
\text { Rhône et l'Ouest des Alpes-Maritimes. Ses populations sont menacées } \\
\text { par l'urbanisation, l'intensification de l'agriculture ou la fermeture des } \\
\text { milieux. Vulnérable sur la Liste rouge nationale. II pourrait bénéficier des } \\
\text { lentilles cultivées en agriculture biologique. }\end{array}$ & $E, F$ & Fort \\
\hline $\begin{array}{l}\text { Zygaena cynarae } \\
\text { (Esper, 1789) }\end{array}$ & $\begin{array}{l}\text { Distribution fractionnée, du Sud-Est de la France jusqu'au massif de } \\
\text { l'Altaï; représentée en France par quatre sous-espèces formant des } \\
\text { métapopulations isolées. Espèce fortement menacée du fait du mitage } \\
\text { de ses habitats par l'urbanisation dans les Alpes-Maritimes et par } \\
\text { l'enfrichement dans ses stations iséroises et varoises. }\end{array}$ & $E, F$ & Majeur \\
\hline $\begin{array}{l}\text { Coenagrion caerulescens } \\
\text { (Boyer de Fonscolombe, } \\
\text { 1838) }\end{array}$ & $\begin{array}{l}\text { Espèce spécialiste des petits cours d'eau méditerranéens et à l'aire de } \\
\text { répartition réduite en France. En danger en France, menacée dans } \\
\text { quatre régions d'après les Listes rouges. Confusion possible avec } \\
\text { C. scitulum (Rambur, 1842) qui a une aire plus large, dont des individus } \\
\text { peuvent accidentellement se rencontrer dans le même milieu. }\end{array}$ & C & Majeur \\
\hline $\begin{array}{l}\text { Coenagrion Iunulatum } \\
\text { (Charpentier, 1840) }\end{array}$ & $\begin{array}{l}\text { Espèce localisée en France sur une petite zone du Massif central dans } \\
\text { des tourbières, mares et étang tourbeux en altitude (550 à } 1250 \mathrm{~m}) \text {. } \\
\text { Ces habitats abritent C. hastulatum (Charpentier, 1825) dont l'enjeu } \\
\text { est moins fort mais reste important, I'aire d'occurrence plus étendue, } \\
\text { et le caractère héliophile moins marqué. Vulnérable en France } \\
\text { et menacé dans trois régions. Confusion possible avec certains } \\
\text { C. hastulatum. }\end{array}$ & $\mathrm{D}, \mathrm{C}$ & Majeur \\
\hline $\begin{array}{l}\text { Coenagrion ornatum } \\
\text { (Selys, 1850) }\end{array}$ & $\begin{array}{l}\text { Espèce localisée en France sur une petite zone du Massif central dans les } \\
\text { suintements prairiaux et petits ruisseaux. Évaluée dans quatre régions, } \\
\text { menacée dans trois, quasi menacée dans une. Confusion possible avec } \\
\text { C. mercuriale (Charpentier, 1840), syntopique. }\end{array}$ & $\mathrm{C}$ & Fort \\
\hline $\begin{array}{l}\text { Coenagrion pulchellum } \\
\text { (Vander Linden, 1825) }\end{array}$ & $\begin{array}{l}\text { Espèce localement abondante en France mais assez disséminée. } \\
\text { Elle fréquente des habitats comme les marais et les étangs bien } \\
\text { végétalisés et d'aspect naturel. La pollution des eaux et la pisciculture } \\
\text { intensive sont des menaces. Vulnérable en France, menacée dans } \\
12 \text { régions et quasi menacée dans six autres. Confusion fréquente } \\
\text { avec C. puella (Linnaeus, 1758) peut-être en lien avec sa relative } \\
\text { rareté perçue. }\end{array}$ & C, D & Fort \\
\hline $\begin{array}{l}\text { Cordulegaster bidentata } \\
\text { Selys, } 1843\end{array}$ & $\begin{array}{l}\text { Endémique européenne souvent confinée aux reliefs, spécialiste } \\
\text { des suintements, sources et petits ruisseaux. Ses habitats sont } \\
\text { sporadiques, de faible taille, donc menacés par les modifications du } \\
\text { régime hydrique et par les activités humaines. Évaluée dans } 12 \text { régions, } \\
\text { menacée dans six, quasi menacé dans trois. Son identification requiert } \\
\text { la capture, mais l'identification des exuvies est facile. }\end{array}$ & C & Fort \\
\hline $\begin{array}{l}\text { Lestes macrostigma } \\
\quad \text { (Eversmann, 1836) }\end{array}$ & $\begin{array}{l}\text { L'espèce a une aire limitée à quelques secteurs des côtes atlantiques } \\
\text { et méditerranéennes ainsi qu'à la Corse. Sténotope, elle est } \\
\text { spécialiste des milieux saumâtres à Bolboschoenus maritimus } \\
\text { (L.) Palla. Les populations sont de taille assez faible et sujettes à } \\
\text { d'importantes variations (éclipses). En danger en France et menacée } \\
\text { dans deux régions d'après les Listes rouges; quasi menacée dans } \\
\text { une troisième. }\end{array}$ & $A$ & Majeur \\
\hline
\end{tabular}


ANNEXE 2. - Suite.

\begin{tabular}{|c|c|c|c|c|}
\hline \multirow{2}{*}{\multicolumn{2}{|c|}{ Espèces à enjeux remarquables }} & \multirow[b]{2}{*}{$\begin{array}{l}\text { Commentaire } \\
\text { Taxon eurosibérien dont l'aire d'occurrence en France est fragmentée. } \\
\text { Spécialiste des tourbières et des étangs bien végétalisés et plutôt } \\
\text { acides du niveau de la mer à } 2000 \text { m. C'est une espèce menacée par } \\
\text { l'alevinage et les fortes densités de poisson. Évaluée dans } 11 \text { régions, } \\
\text { menacée dans 10, presque menacée dans une, éteinte en Limousin. } \\
\text { Identification aisée. }\end{array}$} & \multirow{2}{*}{$\begin{array}{r}\text { EUNIS } \\
\text { D, C }\end{array}$} & \multirow{2}{*}{$\begin{array}{c}\begin{array}{c}\text { Niveau } \\
\text { d'enjeu }\end{array} \\
\text { Fort }\end{array}$} \\
\hline & & & & \\
\hline & $\begin{array}{l}\text { Macromia splendens } \\
\quad \text { (Pictet, 1843) }\end{array}$ & $\begin{array}{l}\text { Endémique franco-ibérique à l'aire d'occurrence limitée en France aux } \\
\text { cours d'eau issus du Massif central et des piémonts pyrénéens. } \\
\text { Jugée «vulnérable» en France et menacée dans ses quatre régions de } \\
\text { présence. Facile à identifier (imagos et exuvies). }\end{array}$ & C & Majeur \\
\hline & $\begin{array}{l}\text { Nehalennia speciosa } \\
\text { (Charpentier, 1840) }\end{array}$ & $\begin{array}{l}\text { Une seule station connue en France à distance d'une autre localité } \\
\text { jurassienne en Suisse. Espèce à faible valence écologique (sténotope) } \\
\text { spécialiste des écocomplexes tourbeux liés à un bas-marais alcalin } \\
\text { (Jacquot 2012). En danger critique en France et en Franche-Comté, } \\
\text { disparue en Rhône-Alpes. Espèce aisée à identifier mais difficile à } \\
\text { observer par sa petite taille. }\end{array}$ & $\mathrm{D}, \mathrm{C}$ & Majeur \\
\hline & $\begin{array}{c}\text { Somatochlora arctica } \\
\text { (Zetterstedt, 1840) }\end{array}$ & $\begin{array}{l}\text { Espèce limitée aux secteurs d'altitude moyenne à élevée (au-dessus de } \\
700 \text { m). Sténotope des tourbières, l'espèce «couvre » aussi I'habitat } \\
\text { d'autres espèces aux enjeux forts ou assez forts: S. alpestris (Selys, } \\
\text { 1840), Aeschna caerulea (Ström, 1783), A. subarctica Walker, 1908, } \\
\text { Leucorrhinia dubia (Vander Linden, 1825) tout en étant plus spécialiste } \\
\text { (jusqu'aux tourbières en voie d'assèchement) et mieux répartie sur } \\
\text { l'ensemble de la France. Quasi menacée en France, menacée dans huit } \\
\text { régions, quasi menacée dans deux autres d'après les Listes rouges. }\end{array}$ & $\mathrm{D}$ & Fort \\
\hline & $\begin{array}{l}\text { Somatochlora } \\
\quad \text { flavomaculata } \\
\text { (Vander Linden, 1825) }\end{array}$ & $\begin{array}{l}\text { Élément eurosibérien dont l'aire d'occurrence en France est fractionnée } \\
\text { et semble exclure la zone méditerranéenne à l'exception de la } \\
\text { Corse. Spécialiste des marais et des zones d'étangs végétalisés en } \\
\text { voie d'atterrissement. Évaluée dans } 18 \text { régions, menacée dans } 11, \\
\text { quasi menacée dans quatre. Identification nécessitant la capture ou } \\
\text { l'observation d'un individu posé. }\end{array}$ & $C, D$ & Fort \\
\hline & $\begin{array}{l}\text { Somatochlora meridionalis } \\
\text { Nielsen, } 1935\end{array}$ & $\begin{array}{l}\text { Taxon est-méditerranéen, limité en France à une étroite portion du Var } \\
\text { et à la Corse. Espèce sténotope inféodée aux petits cours d'eau } \\
\text { parfois intermittents. Quasi menacée en France, en danger en PACA } \\
\text { et presque menacée en Corse. Identification nécessitant la capture ou } \\
\text { l'observation d'un individu posé. }\end{array}$ & C & Majeur \\
\hline & $\begin{array}{l}\text { Stylurus flavipes } \\
\text { (Charpentier, 1825) }\end{array}$ & $\begin{array}{l}\text { Taxon eurasiatique spécialiste de la partie aval des grands cours d'eau. } \\
\text { La reconquête par l'espèce de cours d'eau dont elle était jugée } \\
\text { disparue en fait un indicateur intéressant. Évaluée dans neuf régions, } \\
\text { menacée dans cinq, quasi menacée dans trois. L'identification des } \\
\text { imagos nécessite la capture. Exuvies très caractéristiques. }\end{array}$ & C & Fort \\
\hline & $\begin{array}{l}\text { Sympetrum } \\
\text { depressiusculum } \\
\text { (Selys, 1841) }\end{array}$ & $\begin{array}{l}\text { Espèce très localisée en France. L'accomplissement du cycle de vie } \\
\text { semble nécessiter un assec hivernal et une mise en eau estivale que ce } \\
\text { soit sur des plans d'eau ou des cours d'eau. Peut profiter de structures } \\
\text { anthropogènes réunissant ces conditions (canaux d'irrigation, bassins } \\
\text { d'eaux pluviales). En danger en France et menacée dans trois régions, } \\
\text { et disparue dans deux autres d'après les Listes Rouges. }\end{array}$ & C & Majeur \\
\hline & $\begin{array}{l}\text { Sympetrum vulgatum } \\
\text { (Linnaeus, 1758) }\end{array}$ & $\begin{array}{l}\text { Taxon eurosibérien en déclin sur le territoire français. L'espèce affectionne } \\
\text { les marais et les étangs végétalisés. Ses habitats sont différents de ceux } \\
\text { de la plupart des espèces de montagne et elle constitue un indicateur } \\
\text { intéressant. Évaluée dans } 12 \text { régions, menacée dans cinq, quasi } \\
\text { menacée dans deux. Capture nécessaire à l'identification. }\end{array}$ & C, D & Fort \\
\hline \multirow[t]{2}{*}{ Orthoptères } & $\begin{array}{l}\text { Amedegnatiana vicheti } \\
\text { (Delmas \& Rambier, } \\
\text { 1950) }\end{array}$ & $\begin{array}{l}\text { Espèce strictement endémique de certains massifs calcaires du } \\
\text { Languedoc, listée «en danger d'extinction » sur la Liste rouge } \\
\text { européenne, et en priorité } 1 \text { sur la Liste rouge nationale. Sténotope et à } \\
\text { faible capacité de déplacement, elle habite les pelouses thermophiles } \\
\text { rocailleuses et habitats substeppiques (Delorme 2013). Plusieurs } \\
\text { régressions ponctuelles sont avérées et de fortes menaces existent } \\
\text { localement, en particulier liées aux aménagements photovoltaïques, à } \\
\text { l'urbanisation, à la fermeture des milieux et aux incendies. }\end{array}$ & $\mathrm{F}, \mathrm{E}$ & Majeur \\
\hline & $\begin{array}{l}\text { Anonconotus mercantouri } \\
\text { Galvagni \& Fontana, } \\
2003\end{array}$ & $\begin{array}{l}\text { Espèce micro-endémique de la haute vallée de la Vésubie. Au sein } \\
\text { du genre Anonconotus, cette espèce possède une reconnaissance } \\
\text { taxinomique robuste et facile par des caractères morphologiques } \\
\text { externes. Les capacités de dispersion sont très limitées en raison de } \\
\text { son microptérisme. }\end{array}$ & $E$ & Majeur \\
\hline
\end{tabular}




\begin{tabular}{|c|c|c|c|}
\hline Espèces à enjeux remarquables & Commentaire & EUNIS & $\begin{array}{l}\text { Niveau } \\
\text { d'enjeu }\end{array}$ \\
\hline $\begin{array}{l}\text { Celes variabilis } \\
\qquad(\text { Pallas, 1771) }\end{array}$ & $\begin{array}{l}\text { Distribution générale très morcelée. Vulnérable au niveau européen, } \\
\text { fortement menacée d'extinction sur la Liste rouge nationale. Sténotope, } \\
\text { vit uniquement dans les habitats relictuels que constituent les pelouses } \\
\text { steppiques et rocailleuses. Capacités de dispersion limitées, n’a pas } \\
\text { été revue dans plusieurs stations historiques. }\end{array}$ & , & Majeur \\
\hline $\begin{array}{c}\text { Epacromius tergestinus } \\
\text { (Megerle von Mühlfeld } \\
\text { in Charpentier, 1825) }\end{array}$ & $\begin{array}{l}\text { Distribution large mais très morcelée en Europe comme en France. Le } \\
\text { taxon est divisé en deux sous-espèces spécialisées dans des habitats } \\
\text { distincts: } E \text {. } t \text {. tergestinus (Megerle von Mühlfeld in Charpentier, 1825) } \\
\text { sur les schorres (A2.5) et } E \text {. } t \text {. ponticus (Karny, 1907) sur les iscles des } \\
\text { torrents de montagne (C3.62). L'espèce est classée EN en Europe, } \\
\text { et en priorité } 1 \text { en France. Des stations historiques des deux sous- } \\
\text { espèces ont déjà disparues. }\end{array}$ & $A, C$ & Majeur \\
\hline $\begin{array}{l}\text { Ephippiger provincialis } \\
\quad \text { (Yersin, 1854) }\end{array}$ & $\begin{array}{l}\text { Espèce endémique de Provence, présentant de faibles capacités de } \\
\text { dispersion (microptère) et non retrouvée dans plusieurs stations } \\
\text { historiques. II s'agit d'une espèce menacée, évaluée comme vulnérable } \\
\text { sur la Liste rouge européenne et sur la Liste rouge régionale PACA. }\end{array}$ & $\mathrm{F}, \mathrm{E}$ & Fort \\
\hline $\begin{array}{l}\text { Epipodisma pedemontana } \\
\text { (Brunner von Wattenwyl, } \\
\text { 1825) }\end{array}$ & $\begin{array}{l}\text { Espèce subendémique des Alpes occidentales, dont la présence est } \\
\text { relictuelle en France. Elle est considérée comme «quasi menacé » sur } \\
\text { Liste rouge européenne et fortement menacée en France. Ce taxon } \\
\text { affectionne les rocailles, éboulis et pelouses rases de haute altitude. }\end{array}$ & $\mathrm{H}, \mathrm{E}$ & Fort \\
\hline $\begin{array}{l}\text { Gampsocleis glabra } \\
\text { (Herbst, 1786) }\end{array}$ & $\begin{array}{l}\text { Espèce à large distribution mais en forte régression dans une grande } \\
\text { partie de son aire. Classée «vulnérable» en Europe, et fortement } \\
\text { menacée en France d'où elle a disparue de nombreuses régions et } \\
\text { localités du Nord et de l'Ouest, ainsi que du Midi méditerranéen. Elle } \\
\text { est sténotope dans des pelouses sèches sub-steppiques et quelques } \\
\text { landes à bruyères peu pertubées. }\end{array}$ & $E, F$ & Majeur \\
\hline $\begin{array}{c}\text { Gomphocerippus } \\
\text { armoricanus } \\
\text { Defaut, } 2015\end{array}$ & $\begin{array}{l}\text { Espèce subendémique dont } 99 \text { \% des populations sont en France. } \\
\text { Sténotope dans les landes humides et sèches à ajoncs et bruyères, } \\
\text { toujours dans des milieux préservés depuis longtemps. Ce taxon est } \\
\text { considéré comme fortement menacé au niveau national, possède } \\
\text { une distribution morcelée et régresse de façon importante localement } \\
\text { (Bétard 2016). Ses capacités de dispersions sont faibles. }\end{array}$ & $\mathrm{F}$ & Fort \\
\hline $\begin{array}{l}\text { Gomphocerippus } \\
\text { cialancensis } \\
\text { (Nadig, 1986) }\end{array}$ & $\begin{array}{l}\text { Espèce microendémique des Alpes Cottiennes (Italie/France), évaluée en } \\
\text { «quasi menacé» au niveau européen. Sténotope et à faible capacité } \\
\text { de dispersion dans les éboulis sub-végétalisé au-dessus } 2000 \mathrm{~m} \\
\text { d'altitude. }\end{array}$ & $\mathrm{H}$ & Fort \\
\hline $\begin{array}{l}\text { Metrioptera buyssoni } \\
\quad \text { (Saulcy, 1887) }\end{array}$ & $\begin{array}{l}\text { Espèce subendémique des Pyrénées centrales, considérée comme } \\
\text { «en danger d'extinction» sur la liste rouge européenne, et comme } \\
\text { fortement menacée en France. Elle affectionne les pelouses et landes } \\
\text { de moyenne altitude. Capacités de dispersion limitées. }\end{array}$ & $E$ & Fort \\
\hline $\begin{array}{l}\text { Omocestus defauti } \\
\quad \text { Sardet \& Braud, } 2007\end{array}$ & $\begin{array}{l}\text { Espèce endémique de Corse où son aire très fragmentée est proche } \\
\text { de celle d'une microendémique. Elle est inféodée aux pelouses } \\
\text { xériques rocailleuses et aux éboulis faiblement végétalisés. Plusieurs } \\
\text { stations sont rendues vulnérables par les incendies ou les projets } \\
\text { d'aménagements éoliens ou photovoltaïques. }\end{array}$ & $\mathrm{E}, \mathrm{H}$ & Fort \\
\hline $\begin{array}{l}\text { Prionotropis azami } \\
\text { Uvarov, } 1923\end{array}$ & $\begin{array}{l}\text { Espèce endémique des massifs calcaires de Provence, à aire très } \\
\text { morcelée et inféodée aux pelouses sèches et caillouteuses sub- } \\
\text { steppiques. L'espèce est évaluée «en danger d'extinction » aux } \\
\text { niveaux européen et régional PACA. Plusieurs stations sont fortement } \\
\text { menacées par la diminution du pâturage, l'extension de vignobles ou } \\
\text { le développement d'infrastructures industrielles, commerciales ou } \\
\text { urbanistiques (Bence 2021). Squamiptère, l'espèce n'a qu'une faible } \\
\text { capacité à la dispersion. }\end{array}$ & $E$ & Majeur \\
\hline $\begin{array}{l}\text { Prionotropis rhodanica } \\
\text { Uvarov, } 1923\end{array}$ & $\begin{array}{l}\text { Espèce micro-endémique des coussouls de Crau (Bouches-du-Rhône), } \\
\text { inscrite comme en danger critique d'extinction au niveau européen et } \\
\text { proche de l'extinction au niveau national. La population de l'espèce est } \\
\text { fortement fragmentée et a été évaluée à moins de } 5000 \text { individus au } \\
\text { total. La réduction de l'aire est dramatique, atteignant } 70 \% \text { au cours } \\
\text { des } 10 \text { dernières années (Hochkirch et al. 2016). Cette régression se } \\
\text { prolonge, y compris dans les secteurs protégés classés en réserve } \\
\text { naturelle nationale. Un plan de gestion conservatoire de cette espèce } \\
\text { est en oeuvre depuis } 2014 \text {. Enfin, l'espèce est squamiptère et possède } \\
\text { de ce fait de faibles capacités de dispersion. }\end{array}$ & $E$ & Majeur \\
\hline
\end{tabular}


ANNEXE 2. - Suite.

\begin{tabular}{|c|c|c|c|}
\hline Espèces à enjeux remarquables & Commentaire & EUNIS & $\begin{array}{c}\text { Niveau } \\
\text { d'enjeu }\end{array}$ \\
\hline $\begin{array}{l}\text { Pseudomogoplistes } \\
\text { vicentae Gorochov, } \\
1996\end{array}$ & $\begin{array}{l}\text { Espèce sténotope à distribution atlantique, inféodée aux platiers rocheux } \\
\text { maritimes et aux cordons de galets adjacents. La distribution est } \\
\text { toujours morcelée et l'espèce est considérée «en danger d'extinction» } \\
\text { en Europe et en priorité } 1 \text { en France. }\end{array}$ & B & Fort \\
\hline $\begin{array}{l}\text { Roeseliana azami } \\
\quad \text { (Finot, 1892) }\end{array}$ & $\begin{array}{l}\text { Espèce endémique de France (Provence et Gard), évaluée comme } \\
\text { vulnérable en Europe, fortement menacée sur la Liste rouge nationale } \\
\text { et «quasi menacé» sur la Liste rouge régionale PACA. Elle fréquente } \\
\text { des milieux mésophiles variés comme des marais, prairies, jonchaies } \\
\text { mais aussi des fossés et milieux plus artificialisés. Malgré cela, } \\
\text { plusieurs stations historiques, y compris récentes, ont disparues suite à } \\
\text { des aménagements. }\end{array}$ & $E$ & Fort \\
\hline
\end{tabular}


ANNEXE 3. - Proposition d'évaluation du risque d'extinction des 15 Chilopodes à enjeux sélectionnés en France métropolitaine selon la méthode Liste rouge (UICN France 2018a). Abréviations: AOO, zone d'occupation; CR, «en danger critique d'extinction»; DD, données insuffisantes; EN, «en danger d'extinction»; EOO, zone d'occurrence; EX, "éteinte»; LC, "préoccupation mineure»; VU, "vulnérable». Pour B(1+2), B2, D2, ab(i,ii,iii) se reporter au guide de l’UICN France (2018a).

\begin{tabular}{lllcccc}
\hline & \multicolumn{5}{c}{ Nombre } & Catégorie \\
Espèces évaluées & EOO réelle & AOO réelle & localités & France & Critères & Remarques \\
\hline Clinopodes & $<100 \mathrm{~km}^{2}$ & $<20 \mathrm{~km}^{2}$ & $\leq 5$ & VU & D2 & Endémique hors zone centrale du Mercantour, pouvant
\end{tabular}

\begin{tabular}{|c|c|c|c|c|c|c|}
\hline $\begin{array}{l}\text { Clinopodes } \\
\text { vesubiensis } \\
\text { Bonato, lorio \& } \\
\text { Minelli, } 2011\end{array}$ & $<100 \mathrm{~km}^{2}$ & $<20 \mathrm{~km}^{2}$ & $\leq 5$ & VU & D2 & $\begin{array}{l}\text { Endémique hors zone centrale du Mercantour, pouvant } \\
\text { être affectée par une menace le conduisant rapidement } \\
\text { vers CR: surpiétinement et eutrophisation dues au } \\
\text { surpâturage, urbanisation. Secteur bien exploré, } \\
\text { attestant de sa répartition très restreinte. }\end{array}$ \\
\hline $\begin{array}{l}\text { Cryptops lobatus } \\
\text { Verhoeff, } 1931\end{array}$ & $<5000 \mathrm{~km}^{2}$ & $<2000 \mathrm{~km}^{2}$ & $\leq 10$ & VU & $\begin{array}{c}B(1+2) \\
a b(i, i i, i i i)\end{array}$ & $\begin{array}{l}\text { Distribution très fragmentée en raison de l'anthropisation. } \\
\text { Plusieurs des localités boisées des périodes } 1 \text { et } \\
2 \text { ont disparu: destruction par la déforestation et } \\
\text { l'urbanisation (Géoportail). Pression anthropique forte et } \\
\text { continue dans la majorité de son EOO, vouée à détruire } \\
\text { d'autres stations sans prise de mesures. Tout de même } \\
\text { déclassée en VU au lieu d'EN car AOO réelle estimée } \\
>500 \mathrm{~km}^{2} \text { mais }<2000 \mathrm{~km}^{2} \text {. }\end{array}$ \\
\hline
\end{tabular}

Cryptops umbricus $<5000 \mathrm{~km}^{2}<500 \mathrm{~km}^{2} \quad 10$ à $20 \quad$ LC Verhoeff, 1931

Eupolybothrus
tridentinus

(Fanzago, 1874)

$\begin{aligned} & \text { Geophilus } \\ & \text { persephones } \\ & \text { Bonato \& Minelli, } \\ & 1999\end{aligned}$
$\begin{aligned} & \text { Henia bicarinata } \\ & \text { (Meinert, 1870) }\end{aligned}$

Lithobius $\quad<5000 \mathrm{~km}^{2}<500 \mathrm{~km}^{2} \quad 10$ à $20 \quad$ LC
Cavernicolus
$\begin{aligned} & \text { Fanzago, } 1877 \\ & \text { Lithobius dentatus }>20000 \mathrm{~km}^{2}<2000 \mathrm{~km}^{2} \quad>20 \quad \text { LC }\end{aligned}$

Lithobius dentatus $>20000 \mathrm{~km}^{2}<2000 \mathrm{~km}^{2} \quad>20 \quad$ LC

C. L. Koch, 1844

C

$>500 \mathrm{~km}^{2}$ mais $<2000 \mathrm{~km}^{2}$. de son $\mathrm{EOO}$ et $\mathrm{AOO}$, mais certaines grottes proches du littoral seront probablement menacées à moyen terme en raison de la pression anthropique (urbanisation).

DD Espèce en limite d'aire ouest et récemment découverte

en France, très spécialisée écologiquement mais des secteurs propices et méconnus entre les stations découvertes restent à prospecter. Donc EOO et $\mathrm{AOO}$ jugées sous-évaluées, bien qu'assurément restreintes.

D2 Endémique extrêmement restreinte, pouvant très rapidement basculer vers $C R$ voire EX à la moindre perturbation (trop forte activité spéléologique, dépôts sauvages, etc.).

B2 Distribution sévèrement fragmentée par l'anthropisation.

$\mathrm{ab}(\mathrm{i}, \mathrm{ii}, \mathrm{iii}, \mathrm{iv}) \quad H a l o p h i l e$ bien étudiée et en très forte régression (Iorio et al. 2015, 2020; Geoffroy \& lorio 2019), disparue de toutes ses stations historiques «continentales » sauf une. Forte pression continue sur l'habitat sur tout le littoral méditerranéen de France: aménagement des plages pour le tourisme, nettoyage mécanique, surpiétinement dû à la fréquentation en augmentation en raison de nouveaux cheminements ou modes de transports (cf. vélos électriques à Porquerolles), favorisant l'accès à des plages préservées; création ou extension de ports. Une des localités de 2019 déjà vouée à disparaître à cause d'un aménagement. Quelques plages non prospectées et favorables existent encore potentiellement, mais l'ensemble est nettement $<500 \mathrm{~km}^{2}$ et voué à être rapidement menacé. Nota: habitat littoral très étroit, ce qui renforce le constat établi (occupation réelle/maille = 0,02 km²). Localisée, mais grottes peu menacées en l'état actuel. Surveiller tout de même les éventuels aménagements spéléologiques et/ou la fréquentation trop intensive, la pollution.

Disparitions probables dans plusieurs stations historiques pour ce sylvicole, répartition très fragmentée dans le Nord-Ouest et le Sud-Est. Jugée peu menacée dans le Nord-Est où plusieurs autres stations restent à découvrir dans des massifs forestiers encore inexplorés. Attention à la déforestation, à l'intensité de la sylviculture et au réchauffement climatique qui constituent des menaces sur le long terme.

\begin{tabular}{|c|c|c|c|c|c|c|}
\hline $\begin{array}{l}\text { Lithobius fagniezi } \\
\quad \text { Ribaut, } 1926\end{array}$ & $<500 \mathrm{~km}^{2}$ & $<20 \mathrm{~km}^{2}$ & $\leq 5$ & EN & $\begin{array}{c}B(1+2) \\
a b(i, i i, i i i, i v)\end{array}$ & $\begin{array}{l}\text { Distribution très fragmentée. Troglobionte très localisé, ne } \\
\text { vivant que dans huit stations, dont une déjà disparue } \\
\text { (détruite par l'urbanisation et une carrière) et une autre } \\
\text { visiblement détruite en } 2019 \text { par inondation due à un } \\
\text { projet de carrière. Pression anthropique continue: } \\
\text { urbanisation, carrières, photovoltaïque, fréquentation } \\
\text { touristique. Nombre de localités = } 5 \text { car six stations } \\
\text { restantes mais deux voisines pouvant être affectées par } \\
\text { la même perturbation. }\end{array}$ \\
\hline
\end{tabular}


ANNEXe 3. - Suite.

\begin{tabular}{|c|c|c|c|c|c|c|}
\hline Espèces évaluées & EOO réelle & AOO réelle & $\begin{array}{l}\text { Nombre } \\
\text { localités }\end{array}$ & $\begin{array}{c}\text { Catégorie } \\
\text { France }\end{array}$ & Critères & Remarques \\
\hline $\begin{array}{l}\text { Lithobius pelidnus } \\
\text { Haase, } 1880\end{array}$ & $>20000 \mathrm{~km}^{2}$ & $<2000 \mathrm{~km}^{2}$ & $>20$ & LC & & $\begin{array}{l}\text { Disparitions probables dans plusieurs stations historiques } \\
\text { pour ce sylvicole surtout septentrional. Sensible dans } \\
\text { le Centre et le Nord-Ouest où sa répartition est très } \\
\text { fragmentée, mais peu menacé dans le Nord-Est, } \\
\text { où plusieurs autres stations restent probablement } \\
\text { à découvrir dans des massifs forestiers encore } \\
\text { inexplorés. Attention à la déforestation, à l'intensité de } \\
\text { la gestion forestière et au réchauffement climatique qui } \\
\text { constituent des menaces sur le long terme. }\end{array}$ \\
\hline $\begin{array}{l}\text { Lithobius raffaldii } \\
\quad \text { lorio, } 2009\end{array}$ & $<500 \mathrm{~km}^{2}$ & $<20 \mathrm{~km}^{2}$ & $\leq 5$ & VU & D2 & $\begin{array}{l}\text { Troglobionte endémique très restreinte, pouvant } \\
\text { rapidement basculer vers CR à la moindre perturbation } \\
\text { (trop forte activité spéléologique ou fréquentation } \\
\text { touristique, pollution due à des dépôts sauvages). } \\
\text { Haute-Corse relativement bien prospectée pour les } \\
\text { Chilopodes cavernicoles, attestant de sa répartition. }\end{array}$ \\
\hline $\begin{array}{l}\text { Lithobius ribauti } \\
\quad \text { Chalande, } 1907\end{array}$ & $<5000 \mathrm{~km}^{2}$ & $<500 \mathrm{~km}^{2}$ & $\leq 10$ & DD & & $\begin{array}{l}\text { Probablement peu menacée pour l'instant car ses habitats } \\
\text { semblent avoir peu régressé dans l'aire concernée } \\
\text { (d'après examen éco-paysager), mais dans le détail } \\
\text { la question reste posée (la gestion forestière, par } \\
\text { exemple, peut influer). Espèce très rare, donc difficile } \\
\text { à trouver, malgré la bonne pression de prospection } \\
\text { pyrénéenne. Devra faire l'objet de prospections ciblées } \\
\text { dans les stations historiques pour redéfinir son statut. }\end{array}$ \\
\hline $\begin{array}{l}\text { Lithobius variegatus } \\
\quad \text { Leach, } 1814\end{array}$ & $<20000 \mathrm{~km}^{2}$ & $<500 \mathrm{~km}^{2}$ & $\leq 10$ & VU & $\begin{array}{c}B(1+2) \\
a b(i, i i, i i i, i v)\end{array}$ & $\begin{array}{l}\text { Distribution sévèrement fragmentée, limitée à la frange } \\
\text { atlantique. Sylvicole sensible à la concurrence par } \\
\text { Lithobius forficatus (Linnaeus, 1758) (ce dernier favorisé } \\
\text { par les perturbations) (Lewis 1997). Deux localités des } \\
\text { années } 2000 \text { ont disparu (prospections ciblées en } 2017 \text {, } \\
2018 \text { et } 2019 \text {; É. Texier comm. pers.) à cause d'une } \\
\text { pression sylviculturale récente (nets éclaircissements). } \\
\text { Espèce pourtant facile à détecter à vue. Localités de } \\
\text { la Manche toujours viables (A. Livory, É. Texier comm. } \\
\text { pers.); celle de la Sare non reprospectée, mais sans } \\
\text { doute aussi (aucun changement éco-paysager d'après } \\
\text { Géoportail); EOO réelle estimée à 10516 km². AOO } \\
\text { non retenue car probablement sous-évaluée bien } \\
\text { qu'en régression continue; dans tous les cas jugée } \\
\text { < } 2000 \text { km². Principales menaces: déforestation, } \\
\text { sylviculture trop intensive. }\end{array}$ \\
\hline $\begin{array}{l}\text { Schendyla monodi } \\
\quad \text { (Brolemann, 1924) }\end{array}$ & $<20000 \mathrm{~km}^{2}$ & $<500 \mathrm{~km}^{2}$ & $\leq 10$ & VU & $\begin{array}{l}B(1+2) \\
a b(i i i)\end{array}$ & $\begin{array}{l}\text { Milieux (schorre, haute slikke) nettement approfondis } \\
\text { > } 2010 \text { et très peu avant. Mais espèce halophile très } \\
\text { spécialisée et à répartition sévèrement fragmentée } \\
\text { (Racine \& lorio 2017, 2020); très faible capacité de } \\
\text { dispersion. Habitats étroits, localisés et sensibles, } \\
\text { soumis à pression anthropique croissante et continue } \\
\text { au Sud de la Bretagne (Pays de Loire, Aquitaine), où } \\
\text { les stations sont extrêmement isolées les unes des } \\
\text { autres. Jugée menacée à court terme dans plusieurs } \\
\text { stations: anthropisation des plages due au tourisme, } \\
\text { augmentation de la fréquentation, voire aménagements } \\
\text { portuaires. }\end{array}$ \\
\hline $\begin{array}{l}\text { Tuoba poseidonis } \\
\text { (Verhoeff, 1901) }\end{array}$ & $<20000 \mathrm{~km}^{2}$ & $<500 \mathrm{~km}^{2}$ & $\leq 5$ & EN & $\begin{array}{c}\text { B2 } \\
a b(i, i i, i i i, i v)\end{array}$ & $\begin{array}{l}\text { Cas complètement identique à Henia bicarinata, mais } \\
\text { disparue de toutes ses stations historiques sans } \\
\text { exception. Encore plus spécialisé et plus localisé } \\
\text { qu'H. bicarinata (lorio et al. 2020). }\end{array}$ \\
\hline
\end{tabular}


ANNEXE 4. - Liens des études d'impact consultées en libre accès sur internet.

\section{Liens}

http://www.vaucluse.gouv.fr/IMG/pdf/3.1_diagnostic_ecologique.pdf

http://www.loir-et-cher.gouv.fr/content/download/10634/68856/file/3_Etude_impact\%20Vol\%20I.pdf

http://www.oise.gouv.fr/content/download/53415/329051/file/3-Etude\%20ecologique.pdf

http://www.drome.gouv.fr/lMG/pdf/04-etude_impact_-_annexe_6-7-8.pdf

https://www.cotes-darmor.gouv.fr/content/download/32540/230400/file/1-Etude-FF.pdf

http://webissimo.developpement-durable.gouv.fr/IMG/pdf/formulaire_kpark_cordon-littoral_hyeres_autorise_ complet_cle1116ed.pdf

https://www.mayenne.gouv.fr/content/download/33936/245994/file/5b\%20Etude\%20Faune\%20Flore\%20 Milieux\%20Naturels.pdf

https://www.eure.gouv.fr/content/download/26868/179222/file/5-Etude\%20\%C3\%A9cologique\%200GE\%20 et\%20Geoplus.pdf

https://www.eure.gouv.fr/content/download/26868/179222/file/5-Etude\%20\%C3\%A9cologique\%200GE\%20 et\%20Geoplus.pdf

http://www.var.gouv.fr/IMG/pdf/6.4_ei_vaulongue_et_aspe_v3_210618_annexe3_vnei_vaulongue.pdf

http://www.vaucluse.gouv.fr/IMG/pdf/6_-_etude_faune-flore.pdf

https://www.nord.gouv.fr/content/download/41718/286992/file/07\%20-\%20Etude\%20faune\%20-\%20flore.pdf

http://www.pyrenees-orientales.gouv.fr/index.php/content/download/18936/148328/file/5a-quaiDezoums_ dossierEP_pi\%C3\%A8ce4_Etude+impacts_Ipartie1.pdf; http://www.pyrenees-orientales.gouv.fr/index.php/ content/download/18943/148356/file/8c-quaiDezoums_dossier+EP_pi\%C3\%A8ce6_d\%C3\%A9rog+especes_ partie3.pdf

http://www.gers.gouv.fr/content/download/24362/174263/file/7\%20-\%20Volet\%20Naturel\%20.pdf

http://www.valserhone.fr/wp-content/uploads/2017/05/7.1-Etude-\%c2\%aecologique-Lancrans-mars-17.pdf

http://www.corse-du-sud.gouv.fr/IMG/pdf/11-annexe_1-vnei_ambiegna_biotope.pdf

http://www.paca.developpement-durable.gouv.fr/IMG/pdf/f09318p0149_diagnostic.pdf

http://documents.projets-environnement.gouv.fr/2018/11/15/199589/199589_FEl.pdf

http://www.web-en-royans.fr/documents/2018_Petits-Goulets_RD518_Rapport.pdf

http://www.savoie.gouv.fr/content/download/32846/238752/file/2019.07.19\%20-\%20Etude\%20 d\%27impact\%20\%20Ecrin\%20Blanc_la\%20Rosi\%C3\%A8re\%20VF.pdf

http://www.loiret.gouv.fr/content/download/28430/214614/file/2016.01.29_Etude-impact_CECNA.pdf

https://www.mairie-tournefeuille.fr/sites/default/files/annexe-1-apexe-volet-faune-flore-janvier-2013.pdf

http://www.paca.developpement-durable.gouv.fr/IMG/pdf/f09317p0166_volet_naturel_crottes.pdf

http://www.moulins-les-metz.fr/wp-content/uploads/Diagnostic-environnemental-MOULLINS-version-deseptembre-2015-Tome-1.pdf

http://www.grand-est.developpement-durable.gouv.fr/IMG/pdf/dossier-3.pdf

http://www.charente.gouv.fr/content/download/21553/143663/file/6.\%20Etude\%20d\%27Impact\%20NERSAC\%20 partie\%203.pdf

http://www.alpes-de-haute-provence.gouv.fr/content/download/9569/54050/file/Etude\%20Impact_Coteau\%20 de\%20rousset_P2.pdf

http://www.cote-dor.gouv.fr/IMG/pdf/annexe_5_-_etude_ecologique_ecotope_2016.pdf

http://www.doubs.gouv.fr/content/download/22503/150871/file/Etude+d\%27impact+partie+4.pdf

https://www.herault.gouv.fr/content/download/31906/217840/file/002\%20-\%2020130903\%20-\%20CS\%20

La\%20Fenasse\%20-\%20EIE\%20-\%20Volume\%202\%20-\%20VNEI.pdf\#page=1\&zoom=auto,-158,848

https://www.territoire-de-belfort.gouv.fr/content/download/13433/105623/file/PDFsamTMPbufferFFQPS1.pdf

http://www.paca.developpement-durable.gouv.fr/IMG/pdf/1_projet_Voltalia_La_Barben.pdf

https://www.alpes-de-haute-provence.gouv.fr/index.php/content/download/8902/49989/file/5Etudes\%20 sp\%c3\%a9cifiques\%20partie\%201pdf.pdf

http://www.landes.gouv.fr/IMG/pdf/2018_ba009_etude-incidence-mn_2019-05-23.pdf

http://www.aude.gouv.fr/IMG/pdf/ICPE_Vol-_2_Partie2_cle0a1df2.pdf; http://www.aude.gouv.fr/IMG/pdf/ICPE_ Vol-_2_Partie3_cle0d1e61.pdf

http://www.gard.gouv.fr/content/download/28120/200633/file/PC\%20\%C3\%A9tude\%20d\%27impact\%20 environnemental.pdf

http://www.bas-rhin.gouv.fr/content/download/22791/162998/file/DDAE_KARCHER_p9a.pdf

http://webissimo-ide.developpement-durable.gouv.fr/IMG/pdf/annexe_7_etude_faune_flore_cle181627.pdf

http://webissimo.developpement-durable.gouv.fr/IMG/pdf/annexe_cle13e2d2-3.pdf

http://www.bouches-du-rhone.gouv.fr/index.php/content/download/27886/166267/file/PC11\%20Etude\%20 d\%27impact\%20la\%20Feuillane\%20.pdf

http://www.gard.gouv.fr/content/download/20488/151944/file/5_VOLET_NATUR

https://www.aisne.gouv.fr/content/download/7560/41312/file/Rainette_ARF_Vendeuil_octobre2012_v4.pdf

http://lien.herault.fr/documents/Documents/LIEN-Volet_Naturel_de_ I_Etude_d_Impact.pdf

http://www.haute-saone.gouv.fr/content/download/28071/197872/file/19-161\%20TERNUAY\%20Etude\%20 d\%27impact1.pdf

https://www.alpes-maritimes.gouv.fr/content/download/24129/205703/file/1e\%20Etude\%20impact\%20\%20 piece\%203.pdf

https://participer.loire-atlantique.fr/uploads/decidim/attachment/file/601/Investigations_environnementales_ compressed_1_.pdf

http://www.isere.gouv.fr/content/download/32409/241867/file/Annexe

http://www.vosges.gouv.fr/content/download/11675/94288/file/3-3\%20Impact\%20XERTIGNY.pdf

http://www.alpes-de-haute-provence.gouv.fr/content/download/19213/112875/file/Volet\%20naturel\%20\&\%20 N2000\%20-\%20Ste-Tulle\%20le\%20Moulin.pdf

https://smitu.fr/wp-content/uploads/2018/08/Annexe-10_-Etude-Biotope-1.pdf

Dernière consultation

Septembre 2020

Septembre 2020

Septembre 2020

Septembre 2020

Septembre 2020

Septembre 2020

Septembre 2020

Septembre 2020

Septembre 2020

Septembre 2020

Septembre 2020

Septembre 2020

Septembre 2020

Septembre 2020

Septembre 2020

Septembre 2020

Septembre 2020

Septembre 2020

Septembre 2020

Septembre 2020

Septembre 2020

Septembre 2020

Septembre 2020

Septembre 2020

Septembre 2020

Octobre 2020

Octobre 2020

Octobre 2020

Octobre 2020

Octobre 2020

Octobre 2020

Octobre 2020

Octobre 2020

Octobre 2020

Octobre 2020

Octobre 2020

Octobre 2020

Octobre 2020

Octobre 2020

Octobre 2020

Octobre 2020

Octobre 2020

Octobre 2020

Octobre 2020

Octobre 2020

Octobre 2020

Octobre 2020

Octobre 2020

Octobre 2020

Octobre 2020 
ANNEXE 5. - Sources consultées pour l'évaluation approximative du nombre de données disponibles en France fin 2020 sur les papillons diurnes. Ce tableau ne vise qu'à fournir un aperçu approximatif du nombre de données disponibles au niveau national fin 2020. Les sources étant hétérogènes, cette estimation est forcément incomplète. Elle donne tout de même un ordre de grandeur sur ce paramètre.

\begin{tabular}{|c|c|c|}
\hline Région concernée & $\mathbf{N}$ données & Source(s) \\
\hline Alsace & 150000 & Faune Alsace 2020 (S. Lethuillier, comm. pers.) \\
\hline Aquitaine & 303289 & Faune Aquitaine 2020 \\
\hline Auvergne & 216906 & Faune Auvergne 2020 \\
\hline Basse-Normandie & 35814 & Base de données du GRETIA consultée en octobre 2020 (A. Racine, comm. pers.) \\
\hline Haute-Normandie & 35084 & Lorthiois 2015 \\
\hline Bourgogne & 142946 & Société d'Histoire naturelle d'Autun 2015 \\
\hline Bretagne & 120000 & Buord et al. 2017 \\
\hline Centre & 107512 & Faune Cher 2020; Faune Touraine 2020; Archaux et al. 2016 \\
\hline Champagne-Ardennes & 159069 & Faune Champagne-Ardennes 2020 \\
\hline Corse & 8000 & M.-C. Ruiz (comm. pers.) \\
\hline Franche-Comté & 232377 & Frédéric Morra (comm. pers.) \\
\hline Île-de-France & 100400 & Dewulf \& Houard 2016 \\
\hline Limousin & $>40300$ & Société entomologique du Limousin 2020 \\
\hline Lorraine & 46277 & Faune Lorraine 2020 \\
\hline Nord-Pas-de-Calais & 72805 & Inventaire national du Patrimoine naturel 2020 \\
\hline $\begin{array}{l}\text { Midi-Pyrénées et } \\
\text { Languedoc-Roussillon }\end{array}$ & 600000 & Louboutin et al. 2019 \\
\hline PACA & 680000 & Bence \& Richaud 2020 \\
\hline Pays de la Loire & 280771 & Guilloton 2018; Naturalistes angevins 2020; Barrier et al. 2015 \\
\hline Picardie & 50777 & Picardie Nature 2016 \\
\hline Poitou-Charentes & 262000 & Cotrel 2019 \\
\hline Rhône-Alpes & 425648 & Flavia APE 2018 \\
\hline TOTAL & 4069975 & \\
\hline
\end{tabular}


ANNEXE 6. - Sources consultées pour l'évaluation approximative du nombre de données disponibles en France fin 2020 sur les Orthoptères. Ce tableau ne vise qu'à fournir un aperçu approximatif du nombre de données disponibles au niveau national fin 2020. Les sources étant hétérogènes, cette estimation est forcément incomplète. Elle donne tout de même un ordre de grandeur sur ce paramètre.

\begin{tabular}{|c|c|c|}
\hline Région concernée & $\mathbf{N}$ données & Source(s) \\
\hline Alsace & 41000 & IMAGO 2017 \\
\hline Aquitaine & 30189 & Faune Aquitaine 2020 \\
\hline Auvergne & 75509 & Faune Auvergne 2020 \\
\hline $\begin{array}{c}\text { Basse-Normandie et } \\
\text { Haute-Normandie }\end{array}$ & 36000 & Stallegger 2019 \\
\hline Bourgogne & 15600 & Société d'Histoire naturelle d'Autun 2020 \\
\hline Bretagne & $>34000$ & Bretagne Vivante 2018 \\
\hline Centre & 57540 & Inventaire national du Patrimoine naturel/Open Obs 2020 \\
\hline Champagne-Ardennes & 34615 & Faune Champagne-Ardennes 2020 \\
\hline Corse & 6430 & Inventaire national du Patrimoine naturel/Open Obs 2020 \\
\hline Franche-Comté & 71778 & Dehondt 2019 \\
\hline Île-de-France & 40000 & ARB Île-de-France 2020 \\
\hline Limousin & 32554 & Barataud 2019 \\
\hline Lorraine & 8340 & Faune Lorraine 2020 (S. Lethuillier, comm. pers.) \\
\hline Nord-Pas-de-Calais & 17090 & Inventaire national du Patrimoine naturel/Open Obs 2020 \\
\hline Midi-Pyrénées & 18893 & BAZNAT 2020 \\
\hline Languedoc-Roussillon & 40206 & Faune Languedoc-Roussillon 2020 \\
\hline PACA & 200000 & Bence 2018 \\
\hline Pays de la Loire & $>30690$ & Biodiv' Pays de la Loire 2020 \\
\hline Picardie & 23081 & Picardie Nature 2016 \\
\hline Poitou-Charentes & 107453 & Poitou-Charentes Nature 2019 \\
\hline Rhône-Alpes & 115671 & Sardet 2018 \\
\hline TOTAL & 1036189 & \\
\hline
\end{tabular}




\section{TABLE DES MATIÈRES}

Introduction

Matériel et méthodes

Examen des études d'impact 47

Proposition d'une sélection de groupes d'Arthropodes continentaux et synthèses des connaissances 48

Sélection des espèces à enjeux de conservation 48

Résultats

Synthèse des 50 études d'impact concernant les Arthropodes continentaux 49

Synthèse des connaissances biogéographiques, écologiques et de la vulnérabilité globale pour une sélection 51

d'Arthropodes continentaux

Chilopoda

Isopoda Oniscidea

Coleoptera Cerambycidae et Vesperidae («Longicornes») 54

Coleoptera Scarabaeoidea $\quad 55$

Coleoptera Tenebrionidae $\quad 56$

Hemiptera Heteroptera Pentatomoidea $\quad 57$

Lepidoptera «Rhopalocères» et Zygaena 58

Odonata $\quad 59$

$\begin{array}{ll}\text { Orthoptera } & 60\end{array}$

Constat général 61

Sélection de quinze espèces à enjeux forts ou majeurs de conservation pour les groupes étudiés et éléments de 61 synthèse

135 espèces à enjeux remarquables de conservation

Synthèse par grands types d'habitats selon la typologie EUNIS

Espèces réglementaires absentes des forts enjeux, avec zoom sur le cas de Zerynthia polyxena 62

Réexamen critique de trois types d'habitats rencontrés à l'aide des six nouveaux groupes retenus 63

Discussion

Quels habitats et enjeux de conservation reflètent les groupes actuellements évalués dans les études d'impact? 64

Qu'apporterait l'ajout de six autres groupes d'Arthropodes pour la qualité des diagnostics dans les études d'impact? 65

Un cas remarquable: les plages maritimes et leurs milieux annexes (schorres, dunes) (A et B d'EUNIS) 65

Autres éléments de synthèse générale et par type d'habitat 66

La fonction de "parapluie» et les espèces protégées 69

$\begin{array}{ll}\text { Conclusion } & 70 \\ \text { Remerciements }\end{array}$

Remerciements

Références

Annexe 1. - Liste des espèces autochtones d'Arthropodes terrestres à statut réglementaire au(x) niveau(x) national 80 et/ou européen en France (TaxRef V14 et base de connaissance statut).

Annexe 2. - Sélection d'espèces à enjeux remarquables pour les neuf groupes étudiés. 82

Annexe 3. - Proposition d'évaluation du risque d'extinction des 15 Chilopodes à enjeux sélectionnés en France 94 métropolitaine selon la méthode Liste rouge.

Annexe 4. - Liens des études d'impact consultées en libre accès sur internet. 96

Annexe 5. - Sources consultées pour l'évaluation approximative du nombre de données disponibles en France fin 97 2020 sur les papillons diurnes.

Annexe 6. - Sources consultées pour l'évaluation approximative du nombre de données disponibles en France fin 98 2020 sur les Orthoptères. 